\title{
Heegner points and $p$-adic $L$-functions for elliptic curves over certain totally real fields
}

\author{
Chung Pang Mok
}

\begin{abstract}
For an elliptic curve $E$ over $\mathbb{Q}$ satisfying suitable hypotheses, Bertolini and Darmon have derived a formula for the Heegner point on $E$ in terms of the central derivative of the two variable $p$-adic $L$-function associated to $E$. In this paper, we generalize their work to the setting of totally real fields in which $p$ is inert. We also use this generalization to improve the results obtained by Bertolini-Darmon in the case of an elliptic curve defined over the field of rational numbers.
\end{abstract}

Mathematics Subject Classification (2010). 11G40, 11F33.

Keywords. Hilbert modular forms, $p$-adic $L$-functions, Heegner points.

\section{Contents}

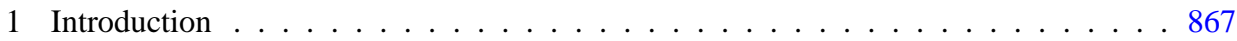

2 Quaternionic automorphic forms . . . . . . . . . . . . . . 871

3 Interpolation of special $L$-values along Hida family . . . . . . . . . . . . . . . . . 896

4 Heegner points on Shimura curves . . . . . . . . . . . . . . . . 905

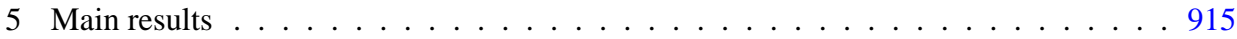

6 Base change arguments . . . . . . . . . . . . . . . . . . . 923

7 Appendix I . . . . . . . . . . . . . . . . . . . . . . . . . 933

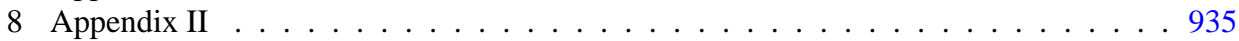

References . . . . . . . . . . . . . . . . . . . . . . . 943

\section{Introduction}

In this paper, we give a partial generalization of the main results of Bertolini-Darmon [4] to elliptic curves over certain totally real fields.

Let $F$ be a totally real field, and $E / F$ be a modular elliptic curve, i.e. associated with a cuspidal Hilbert eigenform $\mathbf{f}_{E}$ over $F$ of parallel weight two.

Let $p$ be a fixed odd prime. We assume that $p$ is inert in $F$, with $\mathfrak{p}=p \mathcal{O}_{F}$ the unique prime of $F$ above $p$. Denote by $L_{p}(s, E / F)$ the $p$-adic $L$-function of $E / F$. 
Suppose that $E$ is split multiplicative at $\mathfrak{p}$. Then by the results of [26], one always has $L_{p}(1, E / F)=0$, called the phenomenon of exceptional zero, and a formula relating $L_{p}^{\prime}(1, E / F)$ and the algebraic part of the $L$-value $L^{\mathrm{alg}}(1, E / F)$ is proved, generalizing that of Greenberg-Stevens [16], [17]. We briefly recall the setup.

Let $\left\{\mathbf{f}_{k}\right\}$ be the Hida family of parallel weights lifting $\mathbf{f}_{E}$, so in particular, for $k \geq 2$, with $k$ sufficiently close to 2 in the weight space, $\mathbf{f}_{k}$ is a cuspidal Hilbert eigenform of parallel weight $k$, and $\mathbf{f}_{2}=\mathbf{f}_{E}$. One has the $p$-adic $L$-function $L_{p}\left(s, \mathbf{f}_{k}\right)$ attached to $\mathbf{f}_{k}$. The results of [26] show that these $p$-adic $L$-functions for different weights can be interpolated to a two-variable $p$-adic $L$-function, i.e., there exists a $p$-adic analytic function $L_{p}(s, k)$ of the two variables $s$ and $k$, such that for $k \geq 2$ an integer, sufficiently close to 2 in the weight space, one has $L_{p}(s, k)=L_{p}\left(s, \mathbf{f}_{k}\right)$. Under the assumption that $E$ is split multiplicative at $\mathfrak{p}, L_{p}(s, k)$ satisfies the functional equation

$$
L_{p}(k-s, k)=-\epsilon_{E / F} L_{p}(s, k),
$$

where $\epsilon_{E / F}$ is the sign for the functional equation of the complex $L$-function $L(s, E / F)$.

The central critical values for the $p$-adic $L$-functions of the Hida family are given by $L_{p}(k / 2, k)$. We have $\left.L_{p}(k / 2, k)\right|_{k=2}=0$ (exceptional zero), and

$$
\left.\frac{d}{d k} L_{p}(k / 2, k)\right|_{k=2}=\left.\frac{1}{2} \frac{\partial L_{p}(s, 2)}{\partial s}\right|_{s=1}+\left.\frac{\partial L_{p}(1, k)}{\partial k}\right|_{k=2} .
$$

By [26] one has the formula

$$
\left.\frac{\partial L_{p}(1, k)}{\partial k}\right|_{k=2}=\left.\frac{d}{d k} \alpha(\mathfrak{p}, k)\right|_{k=2} \cdot L^{\mathrm{alg}}(1, E / F) ;
$$

here $\alpha(\mathfrak{p}, k)$ is the $U_{\mathfrak{p}}$-eigenvalue of the form $\mathbf{f}_{k}$.

Following Bertolini-Darmon, we treat the case where the order of vanishing of the complex $L$-function of $E / F$ at $s=1$ is exactly one, i.e., $L(1, E / F)=0$, and $L^{\prime}(1, E / F) \neq 0$. The sign $\epsilon_{E / F}=-1$, so from the functional equation, the order of vanishing of $L_{p}(s, 2)=L_{p}(s, E / F)$ at $s=1$ is even, so $\left.\frac{\partial L_{p}(s, 2)}{\partial s}\right|_{s=1}=0$. We also have $\left.\frac{\partial L_{p}(1, k)}{\partial k}\right|_{k=2}=0$ by (1.1). Thus $\left.\frac{d}{d k} L_{p}(k / 2, k)\right|_{k=2}=0$. One is thus led to look at the second derivative of $L_{p}(k / 2, k)$ at $k=2$.

We can now state the main result of this paper. Let $Q_{E}$ be the Tate period of $E / F_{\mathfrak{p}}$. For $\sigma \in \operatorname{Hom}\left(F, \overline{\mathbb{Q}}_{p}\right)$, let $E^{\sigma} / F^{\sigma}$ be the elliptic curve which is split multiplicative at the prime $\mathfrak{p}^{\sigma}$ of $F^{\sigma}$, whose Tate period is given by $Q_{E}^{\sigma}$ and which is obtained from $E / F$ by conjugating with $\sigma$. Let $\log _{E^{\sigma} / F_{\mathfrak{p} \sigma}^{\sigma}}$ be the formal logarithm on $E^{\sigma} / F_{\mathfrak{p}^{\sigma}}^{\sigma}$. Then (see Theorem 5.4 and Corollary 5.6 for the precise statements):

Theorem 1.1. Suppose that $L(1, E / F)=0$ but $L^{\prime}(1, E / F) \neq 0$. In the case where $[F: \mathbb{Q}]$ is odd, assume that $E / F$ is multiplicative at some prime other than $\mathfrak{p}$. Then 
there exists a global point $P \in E(F) \otimes \mathbb{Q}$, non-torsion, and a non-zero rational number $l \in \mathbb{Q}^{\times}$, such that

$$
\left.\frac{d^{2}}{d k^{2}} L_{p}(k / 2, k)\right|_{k=2}=l\left(\sum_{\sigma \in \operatorname{Hom}\left(F, \overline{\mathbb{Q}}_{p}\right)} \log _{\left.E^{\sigma} / F_{p^{\sigma}}^{\sigma}\left(P^{\sigma}\right)\right)^{2} .}\right.
$$

The proof largely follows the method of Bertolini-Darmon (with the exception that in the case $F \neq \mathbb{Q}$, the Shimura curves appearing in the argument are not of PEL type, and have more than one geometric component when the strict class number of $F$ is bigger than one; see Section 4 ). In particular, the global point $P$ turns out to be the trace of a Heegner point, so the non-torsion assertion follows from Zhang's generalization [40] of the Gross-Zagier formula, together with the hypothesis that $L^{\prime}(1, E / F) \neq 0$.

As in [4], the extra hypothesis on the conductor of $E / F$, when $[F: \mathbb{Q}]$ is odd, is forced upon us by the use of the theorems of Jacquet-Langlands, and CerednikDrinfeld.

In the case where $F=\mathbb{Q}$, we are able to establish Theorem 1.1 without this extra hypothesis, thus improving the main result of Bertolini-Darmon.

Theorem 1.2. Suppose that $E / \mathbb{Q}$ is split multiplicative at $p$, and satisfies $L(1, E / \mathbb{Q})=0$ but $L^{\prime}(1, E / \mathbb{Q}) \neq 0$. Then there is a non-zero rational number $l \in \mathbb{Q}^{\times}$and $P \in E(\mathbb{Q}) \otimes \mathbb{Q}$ non-torsion, such that

$$
\left.\frac{d^{2}}{d k^{2}} L_{p}(k / 2, k)\right|_{k=2}=l\left(\log _{E / \mathbb{Q}_{p}}(P)\right)^{2}
$$

To do this, one considers base change to a suitable real quadratic field $F$, and apply Theorem 1.1 to $E / F$. Suitable descent arguments allow one to obtain the result over $\mathbb{Q}$. See Section 6 .

Finally we make some remarks about Theorem 1.1. In the case $F=\mathbb{Q}$, formula (1.2) can be regarded as a formula for a non-torsion rational point on $E$, in terms of the central derivative of the two-variable $p$-adic $L$-function of $E$ (by using $p$-adic exponential map to invert the $p$-adic logarithm). However, when $F \neq \mathbb{Q},(1.2)$ falls short of giving such a formula, due to the cross terms involving $E^{\sigma} / F^{\sigma}$, for $\sigma \in \operatorname{Hom}\left(F, \overline{\mathbb{Q}}_{p}\right)$.

The intrinsic difficulty is that, in the statement of Theorem 1.1, one considers only the Hida family of parallel weights. In future work, we would like to obtain such a formula, by considering Hida families of non-parallel weights. This would involve considering mixed partial derivatives of the several variable-variable $p$-adic $L$-function attached to the Hida family constructed in [2].

Acknowledgement. The author would like to thank Professor Darmon, Professor Hida, Professor Mazur and Professor Ribet for encouragements and their discussions 
of the present work. He would also like to thank Samit Dasgupta for a lot of stimulating discussions, and the referees for various helpful comments. The author was partially supported by the NSF grant \# 0901289.

Notation 1.3. As in the introduction, $p$ is a fixed odd prime which we assume to be inert in the totally real field $F$, with $p$ the unique prime of $\mathcal{O}_{F}$ above $p$. Denote by $d$ the degree of $F$ over $\mathbb{Q}$. Thus the norm of $\mathfrak{p}, \mathcal{N} \mathfrak{p}$, is equal to $p^{d}$, which we will denote by $q$ in the sequel. Denote by $F_{+}^{\times}$the set of totally positive elements of $F$, $\mathbf{A}_{F}$ the ring of adeles of $F$, and by $\widehat{F}$ the ring of finite adeles. We have the adelic norms $|\cdot|_{\mathbf{A}_{F}}$ and $|\cdot|_{\hat{F}}$ on $\mathbf{A}_{F}$ and $\hat{F}$ respectively.

For each prime $\mathfrak{l}$ of $F$, denote by valı the normalized valuation of $F_{\mathfrak{I}}$ (or more generally on the algebraic closure of $F_{\mathfrak{Y}}$ ), whose value on the uniformizer of $\mathcal{O}_{F_{\mathfrak{I}}}$ is one.

In general we will denote by $\mathcal{N}$ the norm operation from $F$ to $\mathbb{Q}$, either over the field itself, their completion, the adeles, or at the level of ideal (the subscripts that occur would indicate the field extensions involved).

Denote by $\chi_{\mathbb{Q}, \text { cycl }}: \operatorname{Gal}(\overline{\mathbb{Q}} / \mathbb{Q}) \rightarrow \mathbb{Z}_{p}^{\times}$the $p$-adic cyclotomic character, and by $\chi_{F \text {,cycl }}$ the restriction of $\chi_{\mathbb{Q}, \text { cycl }}$ to $\operatorname{Gal}(\overline{\mathbb{Q}} / F)$. By class field theory, we will also regard $\chi_{F \text {,cycl }}$ as a continuous $\mathbb{Z}_{p}^{\times}$-valued character on $\hat{F}^{\times} / F_{+}^{\times}=\mathbf{A}_{F}^{\times} / F^{\times} F_{\infty,+}$ (here $F_{\infty,+}$ is the archimedean connected component of $\mathbf{A}_{F}^{\times}$). The class field theory isomorphism is normalized so that $\left.\chi_{F \text {,cycl }}\right|_{\mathcal{O}_{F_{\mathfrak{p}}}}$ is equal to $\mathcal{N}_{F_{\mathfrak{p}} / \mathbb{Q}_{p}}$.

Fix algebraic closures $\overline{\mathbb{Q}}$ and $\overline{\mathbb{Q}}_{p}$ of $\mathbb{Q}$ and $\mathbb{Q}_{p}$ respectively. Denote by $\operatorname{ord}_{p}$ the valuation on $\overline{\mathbb{Q}}_{p}$, normalized by the condition $\operatorname{ord}_{p}(p)=1$. We also fix an embedding of $F_{\mathfrak{p}}$ into $\overline{\mathbb{Q}}_{p}$. Under our assumption that $p$ is inert in $F$, we have $F_{\mathfrak{p}}=\mathbb{Q}_{p^{d}}$, the unramified extension of $\mathbb{Q}_{p}$ of degree $d$.

We fix an embedding of $\overline{\mathbb{Q}}$ into $\overline{\mathbb{Q}}_{p}$ once and for all in this paper. Under this embedding, we can identify $\operatorname{Hom}(F, \overline{\mathbb{Q}})$ with $\operatorname{Hom}\left(F, \overline{\mathbb{Q}}_{p}\right)$, which under the assumption that $p$ is inert in $F$, is equal to $\operatorname{Hom}\left(F_{\mathfrak{p}}, \overline{\mathbb{Q}}_{p}\right)$, so that in particular $\mathcal{N}_{F / \mathbb{Q}}(z)=\mathcal{N}_{F_{\mathfrak{p}} / \mathbb{Q}_{p}}(z)$ for $z \in F$.

In general, for any $x \in F_{\mathfrak{p}}$, and $\sigma: F_{\mathfrak{p}} \hookrightarrow \overline{\mathbb{Q}}_{p}$ an embedding, we let $x^{\sigma} \in \overline{\mathbb{Q}}_{p}$ denote the image of $x$ under $\sigma$.

For any number field $L$, denote by $D_{L}$ the absolute value of the discriminant of $L$ over $\mathbb{Q}$. If $\psi$ is a Hecke character of $L$ of finite order, then by class field theory, $\psi$ can be regarded as a character of $\operatorname{Gal}(\bar{L} / L)$, and we denote by $L^{\psi}$ the finite abelian extension of $L$ cut out by ker $\psi$. The conductor of $\psi$ is noted as $c_{\psi}$. Regarding $\psi$ as taking values in $\overline{\mathbb{Q}}$, we denote, for $h \in \operatorname{Aut}(\overline{\mathbb{Q}})$, the character $h \circ \psi$ as $\psi^{h}$.

Finally, if $\mathbb{S}$ is a group and $\chi$ is a character of $\mathbb{S}$, then for $V$ a representation of $\mathbb{S}$, we denote by $V_{\chi}$ the $\chi$-component of $V$. 


\section{Quaternionic automorphic forms}

2.1. Basic definitions. Let $\mathfrak{n}^{-}$be an integral ideal of $\mathcal{O}_{F}$. We assume throughout the paper the following:

Condition 2.1. The ideal $\mathfrak{n}^{-}$is square-free, relatively prime to $\mathfrak{p}$, and such that the number of prime ideal factors of $\mathfrak{n}^{-}$is congruent to $[F: \mathbb{Q}] \bmod 2$. In particular, $\mathfrak{n}^{-}=\mathcal{O}_{F}$ is allowed if $[F: \mathbb{Q}]$ is even.

Let $B$ be the quaternion algebra over $F$, which is ramified exactly at all the archimedean places, and all places dividing $\mathfrak{n}^{-}$. Note that $B$ exists by Condition 2.1 on $\mathfrak{n}^{-}$. In particular, $B$ is totally definite, that is, $B \otimes_{F, v} \mathbb{R}$ is isomorphic to Hamilton's quaternions, for all the real places $v: F \rightarrow \mathbb{R}$. We denote by $\operatorname{Nrd}_{B / F}$ the reduced norm from $B$ to $F$.

Let $\widehat{B}^{\times}=\left(B \otimes_{F} \widehat{F}\right)^{\times}$be the group of finite adelic points of $B^{\times}$. Given $b \in \widehat{B}^{\times}$, and a place $v$ of $F$, we will denote by $b_{v}$ the component of $b$ at $v$. We will generally identify the finite places of $F$ with prime ideals of $\mathcal{O}_{F}$, so if $v$ corresponds to a prime $\mathfrak{I}$, then we will also write $b_{\mathfrak{l}}$ for the corresponding component of $b$.

Fix an isomorphism of $F_{\mathfrak{p}}$-algebras,

$$
\iota_{\mathfrak{p}}: B_{\mathfrak{p}}=B \otimes_{F} F_{\mathfrak{p}} \rightarrow M_{2}\left(F_{\mathfrak{p}}\right)
$$

which induces an isomorphism of $B_{\mathfrak{p}}^{\times}$and $\mathrm{GL}_{2}\left(F_{\mathfrak{p}}\right)$ (here for any ring $A$, we denote by $M_{2}(A)$ the ring of $2 \times 2$ matrices with coefficients in $A$ ).

Let $\Sigma=\prod_{\mathfrak{r}} \Sigma_{\mathfrak{l}}$ be an open compact subgroup of $\widehat{B}^{\times}$. Assume that the image of $\Sigma_{\mathfrak{p}}$ under $\iota_{\mathfrak{p}}$ is contained in $\mathrm{GL}_{2}\left(\mathcal{O}_{F_{\mathfrak{p}}}\right)$.

Let $M$ be a $\mathbb{Z}_{p}$-module, equipped with a left action of $\iota_{\mathfrak{p}}\left(\Sigma_{\mathfrak{p}}\right)$.

Definition 2.2. An $M$-valued automorphic form on $B^{\times}$of level $\Sigma$ is a function

$$
\Phi: \widehat{B}^{\times} \rightarrow M
$$

that satisfies

$$
\Phi(\gamma b u)=\iota_{\mathfrak{p}}\left(u_{\mathfrak{p}}^{-1}\right) \cdot \Phi(b)
$$

for all $\gamma \in B^{\times}, b \in \widehat{B}^{\times}, u \in \Sigma$. Denote by $S(\Sigma, M)$ the space of $M$-valued forms of level $\Sigma$.

Note that $\Phi \in S(\Sigma, M)$ is determined by its values on a set of representatives of the double coset $B^{\times} \backslash \widehat{B}^{\times} / \Sigma$, which is finite (being both compact and discrete). 
Definition 2.3. For each embedding $\sigma: F_{\mathfrak{p}} \rightarrow \overline{\mathbb{Q}}_{p}$, and any integer $n \geq 0$, let $\operatorname{Sym}_{\sigma}^{n}$ be the $\mathbb{C}_{p}$ vector space of homogeneous polynomials of degree $n$ in the indeterminates $X^{\sigma}, Y^{\sigma}$, with coefficients in $\mathbb{C}_{p}$. Define the right action of $\mathrm{GL}_{2}\left(F_{\mathfrak{p}}\right)$ on $\operatorname{Sym}_{\sigma}^{n}$ by

$$
\begin{aligned}
& (P \mid \gamma)\left(X^{\sigma}, Y^{\sigma}\right) \\
& \quad:=P\left(a^{\sigma} X^{\sigma}+b^{\sigma} Y^{\sigma}, c^{\sigma} X^{\sigma}+d^{\sigma} Y^{\sigma}\right) \quad \text { for } \gamma=\left(\begin{array}{ll}
a & b \\
c & d
\end{array}\right) \in \mathrm{GL}_{2}\left(F_{\mathfrak{p}}\right) .
\end{aligned}
$$

For integers $k \geq 2$, put

$$
\mathscr{B}_{k}=\bigotimes_{\sigma: F_{\mathfrak{p}} \rightarrow \overline{\mathbb{Q}}_{p}} \operatorname{Sym}_{\sigma}^{k-2}
$$

with the tensor product right-action of $\mathrm{GL}_{2}\left(F_{\mathfrak{p}}\right)$. Define $V_{k}$ to be the $\mathbb{C}_{p}$-dual of $\mathscr{B}_{k}$, with the dual left action of $\mathrm{GL}_{2}\left(F_{\mathfrak{p}}\right)$. We call $S\left(\Sigma, V_{k}\right)$ the space of classical automorphic forms on $B^{\times}$of parallel weight $k$, and level $\Sigma$.

Consider the following action of $\hat{F}^{\times}$on $S\left(\Sigma, V_{k}\right)$, where given $z \in \hat{F}^{\times}$, it takes $\Phi \in S\left(\Sigma, V_{k}\right)$ to the form $\Phi^{\prime}$, with $\Phi^{\prime}(b)=\Phi(z b)$ for $b \in \widehat{B}^{\times}$. This action factors through the infinite idele class group

$$
Z_{F}(\Sigma):=\hat{F}^{\times} / \overline{F_{+}^{\times}\left(\hat{\mathcal{O}}_{F}^{\times} \cap \Sigma\right)^{\mathfrak{p}}}
$$

(here the superscript $p$ refers to the removal of the component at the place $p$, while the overline on $F^{\times}\left(\hat{\mathcal{O}}_{F}^{\times} \cap \Sigma\right)^{\mathfrak{p}}$ refers to closure).

We have a natural surjection of $Z_{F}(\Sigma)$ to the finite idele class group $\mathrm{Cl}_{F}(\Sigma)$ :

$$
Z_{F}(\Sigma) \rightarrow \mathrm{Cl}_{F}(\Sigma):=\hat{F}^{\times} / F_{+}^{\times}\left(\hat{\mathcal{O}}_{F}^{\times} \cap \Sigma\right)
$$

whose kernel is given by the image of $\mathcal{O}_{F_{\mathfrak{p}}}^{\times} \cap \Sigma_{\mathfrak{p}}$ in $Z_{F}(\Sigma)$ (here we are following the notation of [21], Section 3, p. 313).

From equation (2.1) and Definition 2.3, we see that the action of $\hat{F}^{\times}$on $S\left(\Sigma, V_{k}\right)$, when restricted to the image of $\mathcal{O}_{F_{\mathfrak{p}}}^{\times} \cap \Sigma_{\mathfrak{p}}$ in $Z_{F}(\Sigma)$, is given by $\chi_{F \text {,cycl }}^{2-k}(z)$.

Definition 2.4. For each character $\eta$ of $\mathrm{Cl}_{F}(\Sigma)$, define

$$
\begin{array}{r}
S\left(\Sigma, V_{k}, \eta\right)=\left\{\Phi \in S\left(\Sigma, V_{k}\right), \Phi(z b)=\chi_{F, \text { cycl }}^{2-k}(z) \eta(z)^{-1} \Phi(b)\right. \\
\text { for all } \left.z \in \hat{F}^{\times}, b \in \widehat{B}^{\times}\right\} .
\end{array}
$$

The character $\eta$ is called the nebentype of the form $\Phi$.

It follows that we have a decomposition

$$
S\left(\Sigma, V_{k}\right)=\bigoplus_{\eta} S\left(\Sigma, V_{k}, \eta\right),
$$

where $\eta$ runs over the characters of $\mathrm{Cl}_{F}(\Sigma)$. 
2.2. Hecke operators. Recall the definition of Hecke operators. For each prime $\mathfrak{I}$ at which $B$ splits, and at which $\Sigma_{\mathfrak{l}}$ is maximal, one can define the Hecke operators $T_{\mathfrak{I}}$ as follows. Fix an isomorphism $\iota_{\mathfrak{I}}: B_{\mathfrak{I}} \rightarrow M_{2}\left(F_{\mathfrak{I}}\right)$ such that $\Sigma_{\mathfrak{I}}$ is identified as $\mathrm{GL}_{2}\left(\mathcal{O}_{F_{\mathfrak{I}}}\right)$. Let $\pi_{\mathfrak{r}}$ be an uniformizer of $\mathcal{O}_{F_{\mathfrak{I}}}$, and let $k_{\mathfrak{l}}$ be the residue field at $\mathrm{I}$. Given a double coset decomposition:

$$
\mathrm{GL}_{2}\left(\mathcal{O}_{F_{\mathrm{I}}}\right)\left(\begin{array}{cc}
\pi_{\mathfrak{I}} & 0 \\
0 & 1
\end{array}\right) \mathrm{GL}_{2}\left(\mathcal{O}_{F_{\mathrm{I}}}\right)=\bigsqcup_{a \in \mathbf{P}^{1}\left(k_{\mathfrak{I}}\right)} \sigma_{a}(\mathfrak{l}) \mathrm{GL}_{2}\left(\mathcal{O}_{F_{\mathrm{I}}}\right) .
$$

Define $T_{\mathfrak{I}}$ on $S(\Sigma, M)$ by the rule:

$$
\left(T_{\mathfrak{l}} \Phi\right)(b)= \begin{cases}\sum_{a \in \mathbf{P}^{1}\left(k_{\mathfrak{l}}\right)} \Phi\left(b \sigma_{a}(\mathfrak{l})\right) & \text { if } \mathfrak{l} \neq \mathfrak{p}, \\ \sum_{a \in \mathbf{P}^{1}\left(k_{\mathfrak{l}}\right)} \sigma_{a}(\mathfrak{l}) \cdot \Phi\left(b \sigma_{a}(\mathfrak{l})\right) & \text { if } \mathfrak{l}=\mathfrak{p} .\end{cases}
$$

In the case of $T_{\mathfrak{p}}$, we need to assume that the action of $\iota_{\mathfrak{p}}\left(\Sigma_{\mathfrak{p}}\right)=\mathrm{GL}_{2}\left(\mathcal{O}_{F_{\mathfrak{p}}}\right)$ on $M$ extends to an action of the semi-group $M_{2}\left(\mathcal{O}_{F_{\mathfrak{p}}}\right) \cap \mathrm{GL}_{2}\left(F_{\mathfrak{p}}\right)$.

If $\mathfrak{l} \neq \mathfrak{p}$ (in addition to the condition that $B$ is split at $\mathfrak{l}$ and $\Sigma_{\mathfrak{l}}$ is maximal), we also define the operator $T_{\mathfrak{I}, \mathrm{l}}$ by (cf. the discussion after definition 2.3):

$$
T_{\mathfrak{l}, \mathfrak{l}} \Phi(b)=\Phi\left(\pi_{\mathfrak{r}} b\right) \quad \text { for } b \in \hat{B}^{\times}
$$

(here $\pi_{\mathfrak{r}}$ is identified as the element of $\hat{F}^{\times} \subset \widehat{B}^{\times}$that is equal to $\pi_{\mathfrak{r}}$ at the place $\mathfrak{l}$ and equal to one at other places). Using the fact that the classes of $\pi_{\mathfrak{r}}$ in $\mathrm{Cl}_{F}(\Sigma)$ for all such $\mathfrak{l}$ exhaust $\mathrm{Cl}_{F}(\Sigma)$, we see that if $\Phi \in S\left(\Sigma, V_{k}\right)$, then $\Phi$ has a nebentype, i.e., lies in one of the component $S\left(\Sigma, V_{k}, \eta\right)$, if and only if it is an eigenvector for all the operators $T_{\mathfrak{Y}, \mathfrak{Y}}$, in which case

$$
T_{\mathfrak{l}, \mathfrak{I}} \Phi=\eta(\mathfrak{l})^{-1} \mathcal{N} \mathfrak{\Upsilon}^{k-2} \Phi .
$$

(Here $\eta(\mathfrak{l}):=\eta\left(\pi_{\mathfrak{l}}\right)$, noting that $\eta$ in unramified at $\mathfrak{l}$. Also note our convention about $\chi_{F, \text { cycl }}$ and the global class field theory isomorphism as in Notation 1.3.)

We next define the Hecke operators at a prime at which the level is not maximal. For simplicity we do this only when the level is given by Iwahori subgroups.

In general if $\mathfrak{I}$ is a prime, $m \geq 1$, then we define the Iwahori subgroups $I_{\mathfrak{I}^{m}}$ and $I_{1, I^{m}}$ of $\mathrm{GL}_{2}\left(\mathcal{O}_{F_{\mathrm{I}}}\right)$ of level $\mathfrak{I}^{m}$ by

$$
\begin{gathered}
I_{\mathfrak{I}^{m}}=\left\{\left(\begin{array}{ll}
a & b \\
c & d
\end{array}\right) \in \mathrm{GL}_{2}\left(\mathcal{O}_{F_{\mathfrak{I}}}\right), c \equiv 0 \bmod \pi_{\mathfrak{I}^{m}}\right\}, \\
I_{1, \mathfrak{I}^{m}}=\left\{\left(\begin{array}{ll}
a & b \\
c & d
\end{array}\right) \in I_{\mathfrak{I}^{m}}, a-1 \equiv 0 \bmod \pi_{\mathfrak{I}^{m}}\right\} .
\end{gathered}
$$

Also put

$$
M_{2}\left(\mathfrak{I}^{m} ; \mathcal{O}_{F_{\mathrm{I}}}\right)=\left\{\left(\begin{array}{ll}
a & b \\
c & d
\end{array}\right) \in M_{2}\left(\mathcal{O}_{F_{\mathfrak{I}}}\right), c \equiv 0 \quad \bmod \pi_{\mathfrak{I}^{m}}\right\},
$$




$$
M_{2}\left(1, \mathfrak{I}^{m} ; \mathcal{O}_{F_{\mathfrak{I}}}\right)=\left\{\left(\begin{array}{ll}
a & b \\
c & d
\end{array}\right) \in M_{2}\left(\mathfrak{I}^{m} ; \mathcal{O}_{F_{\mathfrak{I}}}\right), a \equiv 1 \quad \bmod \pi_{\mathfrak{I}^{m}}\right\} .
$$

Suppose that $\mathfrak{l} \nmid \mathfrak{n}^{-}$, and $\Sigma$ is a level such that $I_{1, \mathfrak{I}^{m}} \subset \Sigma_{\mathfrak{l}} \subset I_{\mathfrak{I}} m$ for some $m \geq 1$ (we identify $\Sigma_{\mathfrak{l}}$ with its image under $\iota_{\mathfrak{l}}$ ). Given a double coset decomposition:

$$
I_{1, \mathfrak{I}^{m}}\left(\begin{array}{cc}
1 & 0 \\
0 & \pi_{\mathfrak{l}}
\end{array}\right) I_{1, \mathfrak{I}^{m}}=\bigsqcup_{a \in k_{\mathfrak{I}}} \hat{\sigma}_{a}(\mathfrak{l}) I_{1, \mathfrak{I}^{m}}
$$

define $U_{\mathfrak{I}}$ on $S(\Sigma, M)$ by the rule

$$
\left(U_{\mathfrak{l}} \Phi\right)(b)= \begin{cases}\sum_{a \in k_{\mathfrak{l}}} \Phi\left(b \hat{\sigma}_{a}(\mathfrak{l})\right) & \text { if } \mathfrak{l} \neq \mathfrak{p} \\ \sum_{a \in k_{\mathfrak{l}}} \hat{\sigma}_{a}(\mathfrak{l}) \cdot \Phi\left(b \hat{\sigma}_{a}(\mathfrak{l})\right) & \text { if } \mathfrak{l}=\mathfrak{p}\end{cases}
$$

In the case of $U_{\mathfrak{p}}(\mathfrak{l}=\mathfrak{p})$, we need to assume that $M$ has an action of the semi-group $M_{2}\left(1, \mathfrak{p}^{m} ; \mathcal{O}_{F_{\mathfrak{p}}}\right) \cap \mathrm{GL}_{2}\left(F_{\mathfrak{p}}\right)$ compatible with that of $\Sigma_{\mathfrak{p}}$.

One can take

$$
\hat{\sigma}_{a}(\mathfrak{l})=\left(\begin{array}{cc}
1 & 0 \\
\tilde{a} \pi_{\mathfrak{l}}^{m} & \pi_{\mathfrak{l}}
\end{array}\right),
$$

where $\tilde{a} \in \mathcal{O}_{F_{\mathrm{I}}}$ maps to $a \in k_{\mathrm{\Upsilon}}$.

In the particular case where $\mathfrak{l}=\mathfrak{p}$ and $\Sigma_{\mathfrak{p}}=I_{\mathfrak{p}}$, the matrices $\hat{\sigma}_{a}=\hat{\sigma}_{a}(\mathfrak{p})$ has the following interpretation (under our assumption on $p$ we can take the uniformizer $\pi_{\mathfrak{p}}$ to be $p$ ): let $\{L(a)\}_{a \in k_{\mathfrak{p}}}$ be the $q=p^{d}$ sub-lattices of $\mathcal{O}_{F_{\mathfrak{p}}} \oplus p \mathcal{O}_{F_{\mathfrak{p}}}$ of index equal to $q$, other than the lattice $p\left(\mathcal{O}_{F_{\mathfrak{p}}} \oplus \mathcal{O}_{F_{\mathfrak{p}}}\right)$. The matrices $\hat{\sigma}_{a}$ satisfy

$$
\begin{aligned}
\hat{\sigma}_{a}\left(\mathcal{O}_{F_{\mathfrak{p}}} \oplus \mathcal{O}_{F_{\mathfrak{p}}}\right) & =\mathcal{O}_{F_{\mathfrak{p}}} \oplus p \mathcal{O}_{F_{\mathfrak{p}}}, \\
\hat{\sigma}_{a}\left(\mathcal{O}_{F_{\mathfrak{p}}} \oplus p \mathcal{O}_{F_{\mathfrak{p}}}\right) & =L(a) .
\end{aligned}
$$

2.3. Choice of levels. In this paper, the level $\Sigma$ is defined by the groups of units of local Eichler orders of $B$. Thus let $a$ be an ideal of $\mathcal{O}_{F}$, relatively prime to $\mathfrak{n}^{-}$. For any prime $\mathfrak{l}$, let $R_{\mathfrak{I}}$ be a local order of $B_{\mathfrak{I}}$ satisfying the condition

$$
R_{\mathfrak{I}}=\text { the (unique) maximal order of } B_{\mathfrak{I}} \text { if } \mathfrak{I} \text { divides } \mathfrak{n}^{-},
$$

resp.

$$
R_{\mathfrak{l}}=\text { an Eichler order of level } \mathfrak{l}^{\text {val }_{\mathfrak{l}}(\mathfrak{a})} \text { if } \mathfrak{l} \text { is prime to } \mathfrak{n}^{-} .
$$

For $\mathfrak{l}$ not dividing $\mathfrak{n}^{-}$, we will assume that under the isomorphism $\iota_{\mathfrak{r}}: B_{\mathfrak{l}} \rightarrow M_{2}\left(F_{\mathfrak{l}}\right)$, the image of $R_{\mathfrak{I}}$ is the subring $M_{2}\left(\mathfrak{l}^{\text {val }_{\mathfrak{l}} \boldsymbol{a}} ; \mathcal{O}_{F_{\mathfrak{I}}}\right)$ of $M_{2}\left(\mathcal{O}_{F_{\mathfrak{I}}}\right)$. Thus we have $\iota_{\mathfrak{I}}\left(R_{\mathfrak{l}}^{\times}\right)=$ $I_{\Gamma^{\text {val }}(a) \text {. }}$

Let $\hat{R}=\prod_{\mathrm{r}} R_{\mathrm{I}}$. Then $R:=B \bigcap \widehat{R}$ is an Eichler order of $B$ of level $a$. 
We will denote by $\Sigma\left(a, \mathfrak{n}^{-}\right)$the level given by $\widehat{R}^{\times}$for the above choices of the local orders $R_{\mathfrak{l}}$. Also put

$$
\Sigma_{1}\left(\mathfrak{a}, \mathfrak{n}^{-}\right)=\left\{u \in \Sigma\left(\mathfrak{a}, \mathfrak{n}^{-}\right), u_{\mathfrak{r}} \in I_{1, \mathfrak{I}^{\text {val }_{\mathfrak{r}} \mathrm{a}}} \text { for all } \mathfrak{l} \mid \mathfrak{a}\right\} .
$$

Note that for the level $\Sigma_{1}\left(\mathfrak{a}, \mathfrak{n}^{-}\right)$, the idele class group $\operatorname{Cl}_{F}\left(\Sigma_{1}\left(\mathfrak{a}, \mathfrak{n}^{-}\right)\right)$is the ray class group $\mathrm{Cl}_{F}(a)$ of conductor a times all the archimedean primes. Similarly the infinite idele class group $Z_{F}\left(\Sigma_{1}\left(\mathfrak{a}, \mathfrak{n}^{-}\right)\right)$is equal to the ray class group $Z_{F}(\mathfrak{a})$ of conductor $a \mathfrak{p}^{\infty}$ times all the archimedean primes (in particular independent of $\left.\mathfrak{n}^{-}\right)$. For the levels $\Sigma\left(\mathfrak{a}, \mathfrak{n}^{-}\right)$we have $\mathrm{Cl}_{F}\left(\Sigma\left(\mathfrak{a}, \mathfrak{n}^{-}\right)\right)=\mathrm{Cl}_{F}\left(\mathcal{O}_{F}\right)=\mathrm{Cl}_{F}$ and $Z_{F}\left(\Sigma\left(\mathfrak{a}, \mathfrak{n}^{-}\right)\right)=Z_{F}\left(\mathcal{O}_{F}\right)=Z_{F}$ (here $\mathrm{Cl}_{F}=\hat{F}^{\times} / F_{+}^{\times} \hat{\mathcal{O}}_{F}^{\times}$is the strict ideal class group of $F$, with similar remark applied to $Z_{F}$ ).

Notation 2.5. For $\eta$ a character of $\mathrm{Cl}_{F}(a)$, we will write $S_{k}\left(a, \mathfrak{n}^{-}, \eta\right)$ for the space $S\left(\Sigma_{1}\left(\mathfrak{a}, \mathfrak{n}^{-}\right), V_{k}, \eta\right)$. If $\eta$ is trivial then it is denoted as $S_{k}\left(\mathfrak{a}, \mathfrak{n}^{-}\right)$.

If $\Phi \in S_{k}\left(a, \mathfrak{n}^{-}, \eta\right)$, then it is easy to check that

$$
\Phi(b u)=\eta(u)^{-1}\left(u_{\mathfrak{p}}^{-1} \cdot \Phi(b)\right) \quad \text { for all } u \in \Sigma\left(a, \mathfrak{n}^{-}\right), b \in \widehat{B}^{\times} .
$$

Here if $u \in \Sigma\left(\mathfrak{a}, \mathfrak{n}^{-}\right)$, and $u_{\mathfrak{I}}=\left(\begin{array}{ll}a_{\mathfrak{I}} & b_{\mathfrak{r}} \\ c_{\mathfrak{I}} & d_{\mathfrak{\Upsilon}}\end{array}\right) \in I_{\mathfrak{I}^{\text {val }} \mathfrak{I} \text { a }}$ for $\mathfrak{l} \mid a$, then $\eta(u)$ is defined to be the value of $\eta$ on the idele that is equal to $a_{\mathfrak{r}}$ at places $\mathfrak{I}$ dividing $a$, and equal to one at the other places.

In particular for levels $\Sigma$ with $\Sigma_{1}\left(\mathfrak{a}, \mathfrak{n}^{-}\right) \subset \Sigma \subset \Sigma\left(\mathfrak{a}, \mathfrak{n}^{-}\right)$we have $S\left(\Sigma, V_{k}\right)=$ $\bigoplus_{\eta} S_{k}\left(\mathfrak{a}, \mathfrak{n}^{-}, \eta\right)$, where $\eta$ ranges over the characters of $\mathrm{Cl}_{F}(\Sigma)$.

For $\Sigma_{1}\left(\mathfrak{a}, \mathfrak{n}^{-}\right) \subset \Sigma \subset \Sigma\left(\mathfrak{a}, \mathfrak{n}^{-}\right)$one can also define the Hecke operators $U_{\mathfrak{l}}$ for $\mathfrak{l} \mid \mathfrak{n}^{-}$acting on $S\left(\Sigma, V_{k}\right)$, using the double coset $\Sigma_{\mathfrak{l}} \omega_{\mathfrak{l}} \Sigma_{\mathfrak{l}}=\omega_{\mathfrak{l}} \Sigma_{\mathfrak{l}}$, with $\omega_{\mathfrak{l}}$ a uniformizer of the maximal order $R_{\mathfrak{l}}$ of $B_{\mathfrak{l}}$ (note that $\Sigma_{\mathfrak{l}}=R_{\mathfrak{l}}^{\times}$), i.e., if $\mathfrak{I} \mid \mathfrak{n}^{-}$, then $\left(U_{\mathfrak{I}} \Phi\right)(b)=\Phi\left(b \omega_{\mathfrak{l}}\right)$.

Suppose now that $\Sigma=\Sigma\left(\mathfrak{a}, \mathfrak{n}^{-}\right)$, and that $\mathfrak{l}$ is a prime that divides $a$. Define the trace operator

$$
\operatorname{Tr}_{\mathfrak{a}^{-1}}^{\mathfrak{a}}: S_{k}\left(\mathfrak{a}, \mathfrak{n}^{-}\right) \rightarrow S_{k}\left(\frac{\mathfrak{a}}{\mathfrak{l}}, \mathfrak{n}^{-}\right)
$$

as follows. Given a form $\Phi \in S_{k}\left(a, \mathfrak{n}^{-}\right)$,

$$
\operatorname{Tr}_{a \mathfrak{l}^{-1}}^{\mathfrak{a}}(\Phi)(g)= \begin{cases}\sum_{r} \Phi\left(g \tau_{r}\right) & \text { if } \mathfrak{l} \neq \mathfrak{p}, \\ \sum_{r} \tau_{r} \cdot \Phi\left(g \tau_{r}\right) & \text { if } \mathfrak{l}=\mathfrak{p} .\end{cases}
$$

Here $\left\{\tau_{r}\right\}$ run over a set of left coset representatives of $I_{\mathfrak{I}^{n-1}}$ modulo $I_{\mathfrak{I}^{n}}$ where $n=\operatorname{val}_{\mathfrak{I}}(\mathfrak{a})$ (if $n=1$ then $I_{\mathfrak{I}^{n-1}}$ is interpreted as $\mathrm{GL}_{2}\left(\mathcal{O}_{F_{\mathfrak{I}}}\right)$ ). For example if $\mathfrak{l}$ divides a exactly (i.e., $n=1)$ then one can take $\left\{\tau_{r}\right\}$ with $r$ indexed by $\mathbf{P}^{1}\left(k_{\mathfrak{l}}\right)$, as

$$
\tau_{r}=\left(\begin{array}{ll}
1 & 0 \\
\tilde{r} & 1
\end{array}\right)
$$


for $r \in k_{\mathfrak{r}}$, and

$$
\tau_{\infty}=\left(\begin{array}{cc}
0 & -1 \\
1 & 0
\end{array}\right) .
$$

A form $\Phi \in S_{k}\left(\mathfrak{a}, \mathfrak{n}^{-}\right)$is said to be new at $\mathfrak{l}$, if

$$
\operatorname{Tr}_{a \mathfrak{I}^{-1}}^{a}(\Phi)=0
$$

Otherwise it is called old at $\mathrm{r}$.

2.4. Measure-valued forms. In this section, we define measure-valued forms. First recall the definition of some relevant Iwasawa type algebras.

Let $\mathfrak{n}^{+}$be an ideal relatively prime to $\mathfrak{p} \mathfrak{n}^{-}$. Consider the level $\Sigma=\Sigma\left(\mathfrak{n}^{+}, \mathfrak{n}^{-}\right)$. In this case $\mathrm{Cl}_{F}(\Sigma)=\mathrm{Cl}_{F}$ and $Z_{F}(\Sigma)=Z_{F}$.

Denote by $\mathbf{G}_{F}$ the kernel from $Z_{F}$ to $\mathrm{Cl}_{F}$ :

$$
1 \rightarrow \mathbf{G}_{F} \rightarrow Z_{F} \rightarrow \mathrm{Cl}_{F} \rightarrow 1 .
$$

Then $\mathbf{G}_{F}$ can be described as follows. Denote by $\mathfrak{c}$ the closure in $\mathcal{O}_{F_{\mathfrak{p}}}^{\times}$of the group of totally positive units of $\mathcal{O}_{F}$. Then

$$
\mathbf{G}_{F}=\mathcal{O}_{F_{\mathfrak{p}}}^{\times} / \mathfrak{c} .
$$

Each of the profinite abelian groups $Z_{F}, \mathbf{G}_{F}$ is a (non-canonical) direct product of its finite torsion part and a $\mathbb{Z}_{p}$-free part. The Leopoldt conjecture predicts that these have $\mathbb{Z}_{p}$-rank one, but we do not need this in the sequel.

Put

$$
\mathfrak{X}_{F}=\operatorname{Hom}_{\mathrm{cts}}\left(\mathbf{G}_{F}, \mathbb{C}_{p}^{\times}\right)
$$

known as the $\left(\mathbb{C}_{p}\right.$-points of the) weight space (in [21], Section 3 , the weight space is defined to be $\operatorname{Hom}_{\text {cts }}\left(Z_{F}, \mathbb{C}_{p}^{\times}\right)$. But as in the sequel [22], the above definition is more natural, especially when one is dealing with forms of non-parallel weight).

Inside $\mathfrak{X}_{F}$ is the set of classical weights defined as follows.

For integer $k \geq 2$, and $\xi$ a finite order character of $\mathbf{G}_{F}$, the pair $(k, \xi)$ defines an element $P_{k, \xi} \in \mathfrak{X}_{F}$, called a classical weight, given by

$$
P_{k, \xi}(a)=\xi(a) \chi_{F, \text { cycl }}(a)^{k-2}=\xi(a) \mathcal{N}_{F_{\mathfrak{p}} / \mathbb{Q}_{p}}(a)^{k-2} \text { for } a \in \mathbf{G}_{F} .
$$

(Note that the norm map

$$
\mathcal{N}_{F_{\mathfrak{p}} / \mathbb{Q}_{p}}: \mathcal{O}_{F_{\mathfrak{p}}}^{\times} \rightarrow \mathbb{Z}_{p}^{\times}
$$

factors through

$$
\left.\mathcal{N}_{F_{\mathfrak{p}} / \mathbb{Q}_{p}}: \mathbf{G}_{F}=\mathcal{O}_{F_{\mathfrak{p}}}^{\times} / \mathfrak{c} \rightarrow \mathbb{Z}_{p}^{\times} .\right)
$$


For the purpose of this paper, it suffices to consider those weights with $\xi$ trivial, in which case we denote the weight by $P_{k}$. As we will see below, the weights $P_{k}$ parametrize family of automorphic forms of parallel weight $k$.

Let $\widetilde{\Lambda}_{F}$ be the completed group algebra of $\mathbf{G}_{F}$ with coefficients in $\mathbb{Z}_{p}$ :

$$
\tilde{\Lambda}_{F}=\mathbb{Z}_{p}\left[\llbracket \mathbf{G}_{F}\right] .
$$

Elements of $\mathfrak{X}_{F}=\operatorname{Hom}_{\mathrm{cts}}\left(\mathbf{G}_{F}, \mathbb{C}_{p}^{\times}\right)$extend by continuity to algebra homomorphisms from $\tilde{\Lambda}_{F}$ to $\mathbb{C}_{p}$.

In the case $F=\mathbb{Q}$, then

$$
\tilde{\Lambda}_{\mathbb{Q}}=\mathbb{Z}_{p} \llbracket \mathbb{Z}_{p}^{\times} \rrbracket .
$$

As usual, let $\Lambda \subset \tilde{\Lambda}_{\mathbb{Q}}$ be the Iwasawa algebra:

$$
\Lambda=\mathbb{Z}_{p}\left[11+p \mathbb{Z}_{p}\right] .
$$

The projection map to the one-units,

$$
\langle\cdot\rangle: \mathbb{Z}_{p}^{\times} \rightarrow 1+p \mathbb{Z}_{p}
$$

induces the projection

$$
\tilde{\Lambda}_{\mathbb{Q}} \rightarrow \Lambda
$$

We denote by $\omega_{F}:=\chi_{F, \text { cycl }}\left\langle\chi_{F, \text { cycl }}\right\rangle^{-1}$, the Teichmüller character of $F$. As a Hecke character its conductor is equal to $p$ times the product of all the archimedean places. We have $\omega_{F}=\omega_{\mathbb{Q}} \circ \mathcal{N}_{F / \mathbb{Q}}$.

Let $\Lambda^{\dagger} \subset \mathbb{C}_{p} \llbracket k-2 \rrbracket$ be the subring consisting of power series (with $\mathbb{C}_{p}$ coefficients) that are convergent in some $p$-adic neighbourhood of 2 . We have the usual embedding

$$
\begin{aligned}
\Lambda \hookrightarrow \Lambda^{\dagger}, \\
{[a] \mapsto\left(k \mapsto a^{k-2}\right) ; }
\end{aligned}
$$

here $[a]$ is the group algebra element associated to $a \in 1+p \mathbb{Z}_{p}$. The image of $\Lambda$ in $\Lambda^{\dagger}$ is called the set of Iwasawa functions. If $\lambda$ is an Iwasawa function, then it converges on the region $\operatorname{ord}_{p}(k-2)>1-\frac{1}{p-1}$. For $\lambda \in \Lambda$, and $k \in \mathbb{Z}_{p}$, we will denote by $\lambda(k)$ the evaluation of $\lambda$ at $k$. Similarly if $\tilde{\lambda} \in \tilde{\Lambda}_{\mathbb{Q}}$, then we denote by $\tilde{\lambda}(k)$ the value $\lambda(k)$, where $\lambda$ is the image of $\tilde{\lambda}$ under the map $\widetilde{\Lambda}_{\mathbb{Q}} \rightarrow \Lambda$.

The norm map (2.4) induces the map on the completed group algebras:

$$
\tilde{\Lambda}_{F} \rightarrow \tilde{\Lambda}_{\mathbb{Q}}
$$

and the weights $P_{k}$ factor through (2.6). (For this reason the weights $P_{k}$ are also referred to as cyclotomic weights.) 
Denote by $\left(\mathcal{O}_{F_{\mathfrak{p}}}^{2}\right)^{\prime}$ the set of primitive elements of $\mathcal{O}_{F_{\mathfrak{p}}}^{2}=\mathcal{O}_{F_{\mathfrak{p}}} \oplus \mathcal{O}_{F_{\mathfrak{p}}}$, i.e., elements such that at least one of the coordinates lies in $\mathcal{O}_{F_{\mathfrak{p}}}^{\times}$. Consider the space of orbits $X:=\mathfrak{c} \backslash\left(\mathcal{O}_{F_{\mathfrak{p}}}^{2}\right)^{\prime}$, where $\mathrm{c}$ acts diagonally. The diagonal action of $\mathcal{O}_{F_{\mathfrak{p}}}^{\times}$on $\left(\mathcal{O}_{F_{p}}^{2}\right)^{\prime}$ induces the action of $\mathbf{G}_{F}$ on $X$.

The space of continuous $\mathbb{C}_{p}$-valued functions on $X$ is equipped with the right action of $\mathrm{GL}_{2}\left(\mathcal{O}_{F_{\mathfrak{p}}}\right)$, given by

$$
(f \mid g)(x, y)=f(a x+b y, c x+d y) \quad \text { for } g=\left(\begin{array}{ll}
a & b \\
c & d
\end{array}\right) \in \operatorname{GL}_{2}\left(\mathcal{O}_{F_{\mathfrak{p}}}\right) \text {. }
$$

Let $\mathscr{D}_{*}$ be the corresponding space of measures, i.e., its continuous $\mathbb{C}_{p}$-dual. $\mathscr{D}_{*}$ inherits the dual action of $\mathcal{O}_{F_{\mathfrak{p}}}^{\times}$and $\mathrm{GL}_{2}\left(\mathcal{O}_{F_{\mathfrak{p}}}\right)$ : for $\mu \in \mathscr{D}_{*}$, and $S$ an open compact subset of $X$,

$$
\begin{gathered}
\int_{\mathcal{S}} f(x, y) d(c \cdot \mu)=\int_{\mathcal{S}} f(c x, c y) d \mu \text { for } c \in \mathcal{O}_{F_{\mathfrak{p}}}^{\times}, \\
\int_{\mathcal{S}} f d(g \cdot \mu)=\int_{g^{-1} \mathcal{S}} f \mid g d \mu \text { for } g \in \mathrm{GL}_{2}\left(\mathcal{O}_{F_{\mathfrak{p}}}\right) .
\end{gathered}
$$

The action of $\mathcal{O}_{F_{\mathfrak{p}}}^{\times}$and hence $\mathbf{G}_{F}$ on $\mathscr{D}_{*}$ extends by continuity to give the structure of a $\widetilde{\Lambda}_{F}$-module on $\mathscr{D}_{*}$.

Let $\mathcal{W}:=F_{\mathfrak{p}}^{2}-\{0,0\}$. Put $Y:=c \backslash \mathcal{W}$, so we have the natural inclusion $X \hookrightarrow Y$. Let $\mathscr{D}$ be the space of compactly supported measures on $Y$. $D$ is equipped with the action of $\mathrm{GL}_{2}\left(F_{\mathfrak{p}}\right)$ similar to the action of $\mathrm{GL}_{2}\left(\mathcal{O}_{F_{\mathfrak{p}}}\right)$ on $\mathcal{D}_{*}$ defined above, i.e., if $\mu^{\prime} \in \mathcal{D}$, and $S^{\prime}$ an open compact subset of $Y$, then for $g^{\prime} \in \mathrm{GL}_{2}\left(F_{\mathfrak{p}}\right)$, and $f^{\prime}$ a continuous function on $Y$,

$$
\int_{\mathcal{S}^{\prime}} f^{\prime} d\left(g^{\prime} \cdot \mu^{\prime}\right)=\int_{\left(g^{\prime}\right)^{-1} \mathcal{S}^{\prime}} f^{\prime} \mid g^{\prime} d \mu^{\prime}
$$

(with $f^{\prime} \mid g^{\prime}$ being given by the same formula as (2.7)).

There is an inclusion map $s: \mathscr{D}_{*} \hookrightarrow \mathscr{D}$ obtained by identifying elements of $\mathscr{D}_{*}$ as measures on $Y$ that are supported on $X$. On the other hand, restriction of measures induces the projection map $p: \mathscr{D} \rightarrow \mathscr{D}_{*}$.

Given $\mu \in \mathscr{D}_{*}$, and $g \in M_{2}\left(\mathcal{O}_{F_{\mathfrak{p}}}\right) \cap \mathrm{GL}_{2}\left(F_{\mathfrak{p}}\right)$, define

$$
g \star \mu:=p(g \cdot s(\mu)) \text {. }
$$

Then it is easy to check that this defines a left action of the semi-group $M_{2}\left(\mathcal{O}_{F_{\mathfrak{p}}}\right) \cap$ $\mathrm{GL}_{2}\left(F_{\mathfrak{p}}\right)$ extending that of $\mathrm{GL}_{2}\left(\mathcal{O}_{F_{\mathfrak{p}}}\right)$ (we denote this action by $\star$ in order to distinguish this from the action of $\mathrm{GL}_{2}\left(F_{\mathfrak{p}}\right)$ on $\mathcal{D}$; indeed $\star$ does not extend to an action 
of $\mathrm{GL}_{2}\left(F_{\mathfrak{p}}\right)$ ). Concretely, given a continuous function $f$ on $X$, and $\delta \subset X$ open compact, element $g \in M_{2}\left(\mathcal{O}_{F_{\mathfrak{p}}}\right) \cap \mathrm{GL}_{2}\left(F_{\mathfrak{p}}\right)$ and $\mu \in \mathscr{D}_{*}$ we have

$$
\int_{S} f d(g \star \mu)=\int_{g^{-1}(S) \cap X} f \mid g d \mu .
$$

Hence $g \star \mu$ is supported on $g(X) \cap X$. In particular if $g \in M_{2}\left(\mathcal{O}_{F_{\mathfrak{p}}}\right) \cap \mathrm{GL}_{2}\left(F_{\mathfrak{p}}\right)$, and $\delta \subset X$ such that $g^{-1} S \subset X$, then for $\mu \in D_{*}$ we have

$$
\int_{S} f d g \star \mu=\int_{g^{-1}(\delta) \cap X} f\left|g d \mu=\int_{g^{-1}(\delta)} f\right| g d \mu=\int_{S} f d(g \cdot s(\mu)) .
$$

Definition 2.6. Let $s \in \mathbb{Z}_{p}$. A function $f$ on $X$ is said to be homogeneous with respect to the weight character $\left\langle\chi_{F, \text { cycl }}\right\rangle^{S}$, if

$$
f(c x, c y)=\left\langle\chi_{F, \text { cycl }}(c)\right\rangle^{s} f(x, y)=\left\langle\mathcal{N}_{F_{\mathfrak{p}} / \mathbb{Q}_{p}}(c)\right\rangle^{s} f(x, y)
$$

for any $c \in \mathcal{O}_{F_{\mathfrak{p}}}^{\times}$.

Definition 2.7. We put

$$
\mathscr{D}_{*}^{\text {cycl }}:=\mathscr{D}_{*} \otimes_{\tilde{\Lambda}_{F}} \tilde{\Lambda}_{\mathbb{Q}},
$$

and similarly

$$
\mathscr{D}_{*}^{\text {cycl, } \uparrow}:=\mathscr{D}_{*}^{\text {cycl }} \otimes_{\mathcal{O}_{\mathbb{C}_{p}} \otimes} \Lambda^{\dagger}
$$

(here $\hat{\otimes}$ is the completed tensor product over $\mathbb{Z}_{p}$ ). Elements of $S(\Sigma, M)$, where

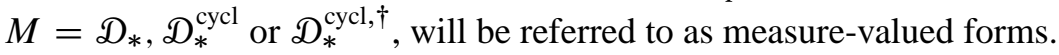

As in Section 2.1 we have an action of $\hat{F}^{\times}$on $S\left(\Sigma, D_{*}\right)$, namely if $z \in \hat{F}^{\times}$, then it takes $\Phi_{\infty} \in S\left(\Sigma, \mathscr{D}_{*}\right)$ to the form $\Phi_{\infty}^{\prime}$, where $\Phi_{\infty}^{\prime}(b)=\Phi_{\infty}(z b)$ for $b \in \widehat{B}^{\times}$. This action factors through $Z_{F}$. On the subgroup $\mathbf{G}_{F}$ of $Z_{F}$, this action is consistent with the action of $\mathbf{G}_{F} \subset \widetilde{\Lambda}_{F}$ on $\mathscr{D}_{*}$ : if $z \in \hat{F}^{\times}$whose class $\bar{z}$ in $Z_{F}$ lies in $\mathbf{G}_{F}$, then

$$
\Phi_{\infty}(z b)=\bar{z}^{-1} \cdot \Phi_{\infty}(b) \text { for } b \in \widehat{B}^{\times} .
$$

Similar remarks apply to $S\left(\Sigma, \mathscr{D}_{*}^{\text {cycl }}\right), S\left(\Sigma, \mathscr{D}_{*}^{\text {cycl }, \dagger}\right)$.

Homogeneous functions with respect to the weight character $\left\langle\chi_{F, \text { cycl }}\right\rangle^{k-2}$ for some $k \in \mathbb{Z}_{p}$ can be integrated against elements of $\mathscr{D}_{*}^{\text {cycl }}$, and also against the elements of $\mathscr{D}_{*}^{\text {cycl, }{ }^{\dagger}}$ if $k$ is $p$-adically sufficiently close to 2 : suppose $\mu=\tilde{\lambda}_{1} \mu_{1}+\cdots+\tilde{\lambda}_{r} \mu_{r}$, with $\mu_{i} \in \mathscr{D}_{*}, \tilde{\lambda}_{i} \in \widetilde{\Lambda}_{\mathbb{Q}}$, and $k \in \mathbb{Z}_{p}$. Then if $f$ is homogeneous of weight $\left\langle\chi_{F, \text { cycl }}\right\rangle^{k-2}$, the formula

$$
\int_{X} f d \mu:=\sum_{i=1}^{r} \tilde{\lambda}_{i}(k) \int_{X} f d \mu_{i}
$$


is well defined.

Elements of $\mathscr{B}_{k}$ give homogeneous functions of weight $\left\langle\chi_{F, \text { cycl }}\right\rangle^{k-2}$ : by linearity, it suffices to consider $P=\otimes_{\sigma} P_{\sigma}$, a pure tensor, with $P_{\sigma} \in \mathrm{Sym}_{\sigma}^{k-2}$ for each $\sigma: F_{\mathfrak{p}} \hookrightarrow \overline{\mathbb{Q}}_{p}$. Then the function

$$
f_{P}(x, y)=\omega_{F}^{2-k}(x) \prod_{\sigma} P_{\sigma}\left(x^{\sigma}, y^{\sigma}\right)
$$

is a homogeneous function of weight $\left\langle\chi_{F, \text { cycl }}\right\rangle^{k-2}$ on $X$. Here $\omega_{F}^{2-k}$ is interpreted as the identity function if $k \equiv 2 \bmod p-1$. Otherwise, if $k$ is not congruent to 2 modulo $p-1$, then we take the convention that $\omega_{F}^{2-k}(x)=0$ if $(x, y) \notin \mathfrak{c} \backslash\left(\mathcal{O}_{F_{\mathfrak{p}}}^{\times} \times \mathcal{O}_{F_{\mathfrak{p}}}\right)$. It can be shown that the association $P \mapsto f_{P}$ is injective.

Definition 2.8. Put $X^{\prime}=\mathfrak{c} \backslash\left(\mathcal{O}_{F_{\mathfrak{p}}}^{\times} \times p \mathcal{O}_{F_{\mathfrak{p}}}\right) \subset X$. Then for integer $k \geq 2$, the specialization to weight $k$ map is given by

$$
\begin{aligned}
\rho_{k}: \mathscr{D}_{*} & \rightarrow V_{k}, \\
\mu & \mapsto\left(P \rightarrow \int_{X^{\prime}} f_{P} d \mu\right) .
\end{aligned}
$$

The same formula defines specialization map $\rho_{k}$ on $\mathscr{D}_{*}^{\text {cycl }}$, and on $\mathscr{D}_{*}^{\text {cycl, } \dagger}$ if $k$ is sufficiently close to $2 p$-adically. The maps $\rho_{k}$ are surjective for each $k \geq 2$.

The specialization map $\rho_{k}$ respects only the action of $I_{1, \mathfrak{p}}$. More precisely, for $k \geq 2$, and $\mu \in \mathscr{D}_{*}, \mathscr{D}_{*}^{\text {cycl }}$ (or $\mathscr{D}_{*}^{\text {cycl, } \dagger}$ if $k$ is $p$-adically sufficiently close to 2 ):

$$
\rho_{k}(u \cdot \mu)=\omega_{F}^{2-k}(u)\left(u \cdot \rho_{k}(\mu)\right) \quad \text { for } u \in I_{\mathfrak{p}} .
$$

In particular it respects the action of $I_{\mathfrak{p}}$ if $k \equiv 2 \bmod p-1$.

Recall that $\Sigma=\Sigma\left(\mathfrak{n}^{+}, \mathfrak{n}^{-}\right)$. Put $\Sigma^{\prime}=\Sigma \cap \Sigma\left(\mathfrak{p}, \mathfrak{n}^{-}\right)=\Sigma\left(\mathfrak{p} \mathfrak{n}^{+}, \mathfrak{n}^{-}\right)$, i.e., $\Sigma^{\prime}$ is the level obtained from $\Sigma$ by replacing $\Sigma_{\mathfrak{p}}=\mathrm{GL}_{2}\left(\mathcal{O}_{F_{\mathfrak{p}}}\right)$ by $I_{\mathfrak{p}}$. Similarly put $\Sigma_{1}^{\prime}=\Sigma \cap \Sigma_{1}\left(\mathfrak{p}, \mathfrak{n}^{-}\right)$. The specialization map $\rho_{k}: \mathscr{D}_{*} \rightarrow V_{k}$ induces

$$
\rho_{k, *}: S\left(\Sigma, D_{*}\right) \rightarrow S\left(\Sigma_{1}^{\prime}, V_{k}\right) .
$$

Similarly we have specialization maps $\rho_{k, *}$ on $S\left(\Sigma, \mathscr{D}_{*}^{\text {cycl }}\right)$, and on $S\left(\Sigma, \mathscr{D}_{*}^{\text {cycl, } \dagger}\right)$ if $k$ is sufficiently close to $2 p$-adically. More precisely. If $\Phi_{\infty} \in S\left(\Sigma, D_{*}^{\text {cycl, } \dagger}\right)$, then there is a $p$-adic disk $U \subset \mathbb{Z}_{p}$ around 2 such that $\Phi_{k}:=\rho_{k, *}\left(\Phi_{\infty}\right)$ is defined for all $k \in U \cap \mathbb{Z}^{\geq 2}$. This can be seen using the finiteness of the double coset $B^{\times} \backslash \hat{B}^{\times} / \Sigma$.

Hence elements of $S\left(\Sigma, D_{*}\right), S\left(\Sigma, \mathscr{D}_{*}^{\text {cycl }}\right)$ and $S\left(\Sigma, \mathscr{D}_{*}^{\text {cycl, } \dagger}\right)$ give rise to $p$-adic families of forms. As usual $p$ appears in the level under specializations, corresponding to the phenomenon of removal of the Euler factor at $\mathfrak{p}$. It is also immediate from the definitions that the map $\rho_{k, *}$ commutes with the Hecke operators $T_{\mathfrak{I}}$ and $T_{\mathfrak{I}, \mathrm{I}}$ for 
$\mathfrak{l} \nmid \mathfrak{p} \mathfrak{n}^{+} \mathfrak{n}^{-}$(and also with the $U_{\mathfrak{l}}$ with $\mathfrak{l} \mid \mathfrak{n}^{+} \mathfrak{n}^{-}$). On the other hand, the action of $T_{\mathfrak{p}}$ on $S\left(\Sigma, \mathscr{D}_{*}\right)$ (defined because $\mathfrak{p} \nmid \mathfrak{n}^{+}$) is actually transferred to the action of $U_{\mathfrak{p}}$ on $S\left(\Sigma_{1}^{\prime}, V_{k}\right)$. In other words, we have the following:

Proposition 2.9. Suppose $\Phi_{\infty} \in S\left(\Sigma, D_{*}\right)$ with $\Sigma$ as above. For $k \geq 2$ integer, put $\Phi_{k}:=\rho_{k, *}\left(\Phi_{\infty}\right)$. Then

$$
\rho_{k, *}\left(T_{\mathfrak{p}} \Phi_{\infty}\right)=U_{\mathfrak{p}} \Phi_{k}
$$

Without interrupting the main reasoning of the paper we refer the reader to Appendix II for the proof.

2.5. $p$-adic deformations of quaternionic eigenforms. With notations as in the previous section, note that the specialization map (for $k$ sufficiently close to two p-adically)

$$
\rho_{k, *}: S\left(\Sigma, D_{*}^{\mathrm{cycl}, \dagger}\right) \rightarrow S\left(\Sigma_{1}^{\prime}, V_{k}\right)
$$

is a map of $\Lambda^{\dagger}$-modules, with the action of $\lambda \in \Lambda^{\dagger}$ on $S_{k}\left(\Sigma_{1}^{\prime}, V_{k}\right)$ being given by multiplication by $\lambda(k)$.

Let $\mathbf{T}$ be the free polynomial algebra over $\mathbb{Z}$ in the symbols $\left\{T_{\mathfrak{l}}\right\}$ for primes $\mathfrak{l} \nmid \mathfrak{n}^{+} \mathfrak{n}^{-}$(in particular for $T_{\mathfrak{p}}$ ), $U_{\mathfrak{l}}$ for $\mathfrak{l} \mid \mathfrak{n}^{+} \mathfrak{n}^{-}$, and $T_{\mathfrak{l}, \mathfrak{l}}$ for $\mathfrak{l} \nmid \mathfrak{p} \mathfrak{n}^{+} \mathfrak{n}^{-}$. The algebra $\mathbf{T}$ acts on $S\left(\Sigma, \mathscr{D}_{*}^{\text {cycl, } \dagger}\right)$ and $S\left(\Sigma_{1}^{\prime}, V_{k}\right)$; we caution here that the symbol $T_{\mathfrak{p}} \in \mathbf{T}$ acts on $S\left(\Sigma_{1}^{\prime}, V_{k}\right)$ via the operator $U_{\mathfrak{p}}$. The map $\rho_{k, *}$ is then a map of $\mathbf{T} \otimes_{\mathbb{Z}} \Lambda^{\dagger}$-modules.

A form $\Phi \in S\left(\Sigma_{1}^{\prime}, V_{k}\right)$ is called an eigenform if it is an eigenvector for the action of $\mathbf{T}$. In this case $\Phi$ has a nebentype and we denote the eigenvalue of $T_{\mathfrak{l}}$ for $\mathfrak{l} \nmid \mathfrak{p} \mathfrak{n}^{+} \mathfrak{n}^{-}$, resp. $U_{\mathfrak{l}}$ for $\mathfrak{l} \mid \mathfrak{p} \mathfrak{n}^{+} \mathfrak{n}^{-}$, as $C(\mathfrak{l}, \Phi)$. In this case the eigenvalues are actually algebraic integers. It is called ordinary at $\mathfrak{p}$, if the $U_{\mathfrak{p}}$-eigenvalue is a $p$-adic unit.

Similarly, a form $\Phi_{\infty} \in S\left(\Sigma, \mathscr{D}_{*}^{\text {cycl, } \dagger}\right)$ is called an eigenform if it is an eigenvector for the action of $\mathbf{T} \otimes \Lambda^{\dagger}$ as a $\Lambda^{\dagger}$-module, i.e., the eigenvalues are in $\Lambda^{\dagger}$ (there is a uniform radius of convergence for the whole package of eigenvalues). Denote by $C\left(\mathfrak{l}, \Phi_{\infty}\right) \in \Lambda^{\dagger}$ the eigenvalue for $T_{\mathfrak{l}}$ when $\mathfrak{l} \nmid \mathfrak{n}^{+} \mathfrak{n}^{-}$, and $U_{\mathfrak{l}}$ if $\mathfrak{l} \mid \mathfrak{n}^{+} \mathfrak{n}^{-}$. The form $\Phi_{\infty}$ is called ordinary at $\mathfrak{p}$ if $C\left(\mathfrak{p}, \Phi_{\infty}\right) \in\left(\Lambda^{\dagger}\right)^{\times}$.

Suppose that $\Phi_{\infty} \in S\left(\Sigma, \mathscr{D}_{*}^{\text {cycl, } \dagger}\right)$ is an eigenform. Then there is a character $\eta_{\infty}$ on $Z_{F}$ with values in $\left(\Lambda^{\dagger}\right)^{\times}$such that $T_{\mathfrak{l}, \mathfrak{l}} \Phi_{\infty}=\eta_{\infty}(\mathfrak{l})^{-1} \Phi_{\infty}$ for $\mathfrak{l} \nmid \mathfrak{p} \mathfrak{n}^{+} \mathfrak{n}^{-}$ (here $\eta_{\infty}(\mathfrak{l})=\eta_{\infty}\left(\pi_{\mathfrak{l}}\right)$ with $\pi_{\mathfrak{l}}$ a uniformizer of $F_{\mathfrak{l}}$; the existence of $\eta_{\infty}$ follows from the density theorem of Cebotarev, which asserts that the classes of $\pi_{\mathfrak{r}}$ in $Z_{F}$ for $\mathfrak{l} \nmid \mathfrak{p} \mathfrak{n}^{+} \mathfrak{n}^{-}$are dense in $\left.Z_{F}\right)$. Assume that $\Phi_{2}$ is not identically zero. Let $U$ be a $p$-adic disk around 2 so that the specializations $\Phi_{k}=\rho_{k, *}\left(\Phi_{\infty}\right)$ are defined for $k \in U \cap \mathbb{Z}^{\geq 2}$ and not identically zero. Then the forms $\Phi_{k}$ are eigenforms. We claim that the nebentype of $\Phi_{k}$ is given by the character $\eta \omega_{F}^{2-k}$ on $\mathrm{Cl}_{F}(\mathfrak{p})$, where 
$\eta=\eta_{2}$ is the character on $\mathrm{Cl}_{F}$ given by the image of $\eta_{\infty}$ under the evaluation at 2 map $\lambda \mapsto \lambda(2)$ for $\lambda \in \Lambda^{\dagger}$. Indeed, we know that in any case $\Phi_{k}$ has a nebentype character $\eta_{k}$ on $\mathrm{Cl}_{F}(\mathfrak{p})$, satisfying

$$
T_{\mathfrak{l}, \mathfrak{I}} \Phi_{k}=\mathcal{N} \mathfrak{l}^{k-2} \eta_{k}(\mathfrak{l})^{-1} \Phi_{k} .
$$

Hence

$$
\eta_{\infty}(\mathfrak{l})(k)=\mathcal{N} \mathfrak{l}^{2-k} \eta_{k}(\mathfrak{l}) \quad \text { for } k \in U \cap \mathbb{Z}^{\geq 2} .
$$

It follows that the element $\langle\mathcal{N} \mathfrak{l}\rangle^{k-2} \cdot \eta_{\infty}(\mathfrak{l})(k) \in \Lambda^{\dagger}$ (here $k$ is a variable) can take only finitely many values as $k$ ranges over $U \cap \mathbb{Z}^{\geq 2}$, hence must be a constant equal to $\eta_{\infty}(\mathfrak{l})(2)=\eta_{2}(\mathfrak{l})$. The claim follows. In particular, when $k \in U \cap \mathbb{Z}^{\geq 2}$ and $k \equiv 2 \bmod p-1$, the nebentype of $\Phi_{k}$ is given by $\eta=\eta_{2}$, and $\Phi_{k}$ is of level $\Sigma\left(\mathfrak{p} \mathfrak{n}^{+}, \mathfrak{n}^{-}\right)\left(\right.$instead of just $\left.\Sigma\left(\mathfrak{n}^{+}, \mathfrak{n}^{-}\right) \cap \Sigma_{1}\left(\mathfrak{p}, \mathfrak{n}^{-}\right)\right)$.

We now state a version of Hida's theory on lifting eigenform to a $p$-adic family, in the style of Greenberg-Stevens [16]:

Theorem 2.10. Let $\Phi=\Phi_{2} \in S\left(\Sigma\left(\mathfrak{p} \mathfrak{n}^{+}, \mathfrak{n}^{-}\right), V_{2}\right)$ be an eigenform that is $\mathfrak{p}$ ordinary, and is new at primes dividing $\mathfrak{n}^{+}$. Then there is an eigenform $\Phi_{\infty} \in$ $S\left(\Sigma\left(\mathfrak{n}^{+}, \mathfrak{n}^{-}\right), D_{*}^{\text {cycl, } \dagger}\right)$ such that $\rho_{2, *}\left(\Phi_{\infty}\right)=\Phi_{2}$.

Theorem 2.10 can be proved using the method of [1] and [21]. For the convenience of the reader we give a sketch of proof in Appendix II.

For $k \in U \cap \mathbb{Z}^{\geq 2}$, the eigenform $\Phi_{k} \in S\left(\Sigma\left(\mathfrak{n}^{+}, \mathfrak{n}^{-}\right) \cap \Sigma_{1}\left(\mathfrak{p}, \mathfrak{n}^{-}\right), V_{k}, \eta \omega_{F}^{2-k}\right)$ corresponds to a unique normalized cuspidal Hilbert eigenform over $F$ under the Jacquet-Langlands' correspondence (for the definitions regarding Hilbert modular forms, we refer to [26], Section 2; here a Hilbert modular form $\mathbf{f}$ is called normalized if the normalized Fourier coefficient $C\left(\mathcal{O}_{F}, \mathbf{f}\right)$, in the notation of [26], Section 2, is equal to one). More precisely, there is a unique normalized cuspidal Hilbert eigenform $\mathbf{f}_{k}$ of parallel weight $k$, level $\mathfrak{p} \mathfrak{n}^{+} \mathfrak{n}^{-}$, that is new at primes dividing $\mathfrak{n}^{+} \mathfrak{n}^{-}$and ordinary at $\mathfrak{p}$, such that the Hecke eigenvalues of $\mathbf{f}_{k}$ and $\Phi_{k}$ with respect to $\mathbf{T}$ coincide (again the symbol $T_{\mathfrak{p}}$ acts as the operator $U_{\mathfrak{p}}$ on $\mathbf{f}_{k}$ ). Remark that for normalized eigenform $\mathbf{f}_{k}$, the eigenvalues of $\mathbf{f}_{k}$ with respect to the Hecke operators $T_{\mathfrak{l}}$ for $\mathfrak{l} \nmid \mathfrak{p} \mathfrak{n}^{+} \mathfrak{n}^{-}$(resp. $U_{\mathfrak{l}}$ if $\left.\mathfrak{l} \mid \mathfrak{p} \mathfrak{n}^{+} \mathfrak{n}^{-}\right)$are given by the normalized Fourier coefficient $C\left(\mathfrak{l}, \mathbf{f}_{k}\right)$. Thus we have $C\left(\mathfrak{l}, \mathbf{f}_{k}\right)=C\left(\mathfrak{l}, \Phi_{k}\right)$ for all primes $\mathfrak{l}$, and the nebentype of $\mathbf{f}_{k}$ and $\Phi_{k}$ coincide.

The family of eigenforms $\left\{\mathbf{f}_{k}\right\}_{k \in U \cap \mathbb{Z} \geq 2}$ forms the set of specializations of a $\Lambda^{\dagger}$ adic form $\mathbf{f}_{\infty}$, called the Hida family lifting $\mathbf{f}_{2}$ (cf. [26], Section 4 ). The $\Lambda^{\dagger}$-adic form $\mathbf{f}_{\infty}$ is determined by the data of its normalized Fourier coefficients $C\left(\mathfrak{m}, \mathbf{f}_{\infty}\right) \in \Lambda^{\dagger}$ for all ideals $\mathfrak{m}$ of $\mathcal{O}_{F}$, and is defined as follows. For $\mathfrak{l}$ a prime put

$$
C\left(\mathfrak{l}, \mathbf{f}_{\infty}\right)=C\left(\mathfrak{l}, \Phi_{\infty}\right)
$$


and define $C\left(\mathfrak{m}, \mathbf{f}_{\infty}\right)$ for other $\mathfrak{m}$ by the formal equality

$$
\sum_{\mathfrak{m}} \frac{C\left(\mathfrak{m}, \mathbf{f}_{\infty}\right)}{\mathcal{N} \mathfrak{m}^{s}}=\prod_{\mathfrak{l}} \frac{1}{1-C\left(\mathfrak{l}, \mathbf{f}_{\infty}\right) \mathcal{N} \mathfrak{l}^{-s}+\eta_{\infty}(\mathfrak{l})^{-1} \mathcal{N} \mathfrak{I}^{-2 s}}
$$

Here $\eta_{\infty}$ is the character on $Z_{F}$ associated to $\Phi_{\infty}$ as above, and $\eta_{\infty}(\mathfrak{l})=\eta_{\infty}\left(\pi_{\mathfrak{l}}\right)$ if $\mathfrak{l} \nmid \mathfrak{p} \mathfrak{n}^{+} \mathfrak{n}^{-}$, and zero otherwise. Equation (2.21) means that $\mathbf{f}_{\infty}$ is an eigenform for the action of Hecke operators acting on $\Lambda^{\dagger}$-adic forms of tame level $\mathfrak{n}^{+} \mathfrak{n}^{-}$, and $\mathbf{f}_{\infty}$ has the property that its weight $k$ specialization is the eigenform $\mathbf{f}_{k}$ of parallel weight $k$ for $k \in U \cap \mathbb{Z}^{\geq 2}$. It is ordinary at $\mathfrak{p}$ (in the sense that $\left.C\left(\mathfrak{p}, \mathbf{f}_{\infty}\right)=C\left(\mathfrak{p}, \Phi_{\infty}\right) \in\left(\Lambda^{\dagger}\right)^{\times}\right)$, which thus defines an algebra homomorphism of Hida's universal ordinary Hecke algebra of tame level $\mathfrak{n}^{+} \mathfrak{n}^{-}$lifting $\mathbf{f}_{2}$ ([21], Section 3). We remark that in [21], Theorem 3.6, one starts with $\mathbf{f}_{2}$ and constructs $\mathbf{f}_{\infty}$ directly on the Hilbert modular side independently of $\Phi_{\infty}$. By loc. cit. the Hida family $\mathbf{f}_{\infty}$ is unique.

In the rest of the paper, the form $\Phi=\Phi_{2}$ to start with is assumed to have trivial nebentype, so that $\Phi \in S_{2}\left(\mathfrak{p n}^{+}, \mathfrak{n}^{-}\right)$, and we only need to look at the weight $k$ specializations $\Phi_{k}$ of $\Phi_{\infty}$, with $k \in U \cap \mathbb{Z}^{\geq 2}$, and $k \equiv 2 \bmod p-1$, so that $\Phi_{k} \in S_{k}\left(\mathfrak{p} \mathfrak{n}^{+}, \mathfrak{n}^{-}\right)$.

2.6. Another description of quaternionic forms. Consider the case where $\Sigma=$ $\Sigma\left(\mathfrak{a}, \mathfrak{n}^{-}\right)$for some ideal $a$ ( $a$ will be either $\mathfrak{n}^{+}$or $\left.\mathfrak{p} \mathfrak{n}^{+}\right)$. The theorem of the norm and the strong approximation theorem (Theorems 4.1 and 4.3 in Chapter 3 of [34]) gives a decomposition

$$
\widehat{B}^{\times}=\bigsqcup_{i=1}^{h} B^{\times} x_{i} B_{\mathfrak{p}}^{\times} \Sigma
$$

where $x_{i} \in \widehat{B}^{\times}$satisfies $\left(x_{i}\right)_{\mathfrak{p}}=1$, and such that the reduced norms $\operatorname{Nrd}_{B / F}\left(x_{i}\right) \in$ $\hat{F}^{\times}$, for $i=1, \ldots, h$, give a complete set of representatives of the strict ideal class group $\mathrm{Cl}_{F}$ of $F$. More precisely if $y \in \widehat{B}^{\times}$, then the unique index $i$ of (2.12) to which $y$ belongs is determined by the condition that the class of $\operatorname{Nrd}_{B / F}\left(y x_{i}^{-1}\right)$ in $\mathrm{Cl}_{F}$ is trivial (note that under our assumption that $p$ is inert in $F$, the image of $F_{\mathfrak{p}}^{\times}$ in $\mathrm{Cl}_{F}$ is trivial).

For $i=1, \ldots, h$ define

$$
\begin{gathered}
\widetilde{\Gamma}_{i}=\widetilde{\Gamma}_{i}\left(\mathfrak{a}, \mathfrak{n}^{-}\right):=\left\{\gamma \in B^{\times}, \gamma_{\mathfrak{l}} \in\left(x_{i}\right)_{\mathfrak{l}} \Sigma_{\mathfrak{l}}\left(x_{i}\right)_{\mathfrak{I}}^{-1} \text { for } \mathfrak{I} \neq \mathfrak{p}\right\}, \\
\Gamma_{i}=\Gamma_{i}\left(\mathfrak{a}, \mathfrak{n}^{-}\right)=\left\{\gamma \in \widetilde{\Gamma}_{i}\left(\mathfrak{a}, \mathfrak{n}^{-}\right), \operatorname{Nrd}_{B / F}(\gamma) \in \mathcal{O}_{F,+}^{\times}\right\} .
\end{gathered}
$$

Here $\mathcal{O}_{F,+}^{\times}=\mathcal{O}_{F}^{\times} \cap F_{+}^{\times}$is the group of totally positive units of $\mathcal{O}_{F}$. Note that $R_{i}:=B \cap x_{i} \hat{R} x_{i}^{-1}$ is an Eichler order of $B$ of level $a$, and $\widetilde{\Gamma}_{i}=R_{i}\left[\frac{1}{p}\right]^{\times}$. If $\gamma \in \widetilde{\Gamma}_{i}$, then $\operatorname{Nrd}_{B / F}(\gamma) \in \mathcal{O}_{F}\left[\frac{1}{p}\right]^{\times} \cap F_{+}^{\times}$. Note also that if $a$ and $a^{\prime}$ are two ideals relatively 
prime to $\mathfrak{n}^{-}$that are equal except at the place $\mathfrak{p}$, then $\widetilde{\Gamma}_{i}\left(\mathfrak{a}, \mathfrak{n}^{-}\right)=\widetilde{\Gamma}_{i}\left(\mathfrak{a}^{\prime}, \mathfrak{n}^{-}\right)$and similarly for the $\Gamma_{i}$. For the $p$-adic constructions we embed $\widetilde{\Gamma}_{i}$ as a subgroup of $B_{\mathfrak{p}}^{\times}$, and hence subgroup of $\mathrm{GL}_{2}\left(F_{\mathfrak{p}}\right)$ via $\iota_{\mathfrak{p}}$. In the following, we will usually omit the explicit reference to the isomorphism $\iota_{\mathfrak{p}}$.

Using (2.12), we have a bijection

$$
\bigsqcup_{i=1}^{h} \widetilde{\Gamma}_{i} \backslash B_{\mathfrak{p}}^{\times} / \Sigma_{\mathfrak{p}} \stackrel{\sim}{\longrightarrow} B^{\times} \backslash \widehat{B}^{\times} / \Sigma,
$$

where for $g \in B_{\mathfrak{p}}^{\times}$, the class of $g$ in $\widetilde{\Gamma}_{i} \backslash B_{\mathfrak{p}}^{\times} / \Sigma_{\mathfrak{p}}$ gets mapped to the class of $x_{i} \cdot g$ in $B^{\times} \backslash \widehat{B}^{\times} / \Sigma$.

Using (2.14), we see that a form $\Phi \in S_{k}\left(\mathfrak{a}, \mathfrak{n}^{-}\right)$can be identified as an $h$-tuples of function $\phi^{1}, \ldots, \phi^{h}$ on $\mathrm{GL}_{2}\left(F_{\mathfrak{p}}\right)$, by the rule: $\phi^{i}(g)=\Phi\left(x_{i} \cdot g\right)$, for $i=1, \ldots, h$. The functions $\phi^{i}$, satisfy:

$$
\begin{aligned}
& \phi^{i}(\gamma g u z) \\
& \quad=\chi_{F, \text { cycl }}^{2-k}(z)\left(u^{-1} \cdot \phi^{i}(g)\right) \text { for } \gamma \in \widetilde{\Gamma}_{i}, g \in \mathrm{GL}_{2}\left(F_{\mathfrak{p}}\right), u \in \Sigma_{\mathfrak{p}}, z \in \widehat{F}^{\times} .
\end{aligned}
$$

Similarly if $\Phi_{\infty} \in S\left(\Sigma\left(\mathfrak{n}^{+}, \mathfrak{n}^{-}\right), \mathscr{D}_{*}^{\text {cycl, } \dagger}\right)$ is as in the statement of Theorem 2.10, then it can be identified as an $h$-tuple $\phi_{\infty}^{1}, \ldots, \phi_{\infty}^{h}$ on $\operatorname{GL}_{2}\left(F_{\mathfrak{p}}\right)$, with $\phi_{\infty}^{i}(g)=$ $\Phi_{\infty}\left(x_{i} \cdot g\right)$. We have

$$
\phi_{\infty}^{i}(\gamma g u z)=\eta_{\infty}(z)^{-1} u^{-1} \phi_{\infty}^{i}(g) \text { for } \gamma \in \widetilde{\Gamma}_{i}, g \in \operatorname{GL}_{2}\left(F_{\mathfrak{p}}\right), u \in \Sigma_{\mathfrak{p}}, z \in \widehat{F}^{\times} .
$$

Note that this description is similar to the description of automorphic forms on $\mathrm{GL}_{2}\left(\mathbf{A}_{F}\right)$, in terms of vectors of Hilbert modular forms, cf. Section 2 of [26].

This description is more convenient for local constructions. On the other hand, the original description as in Definition 2.2 is more convenient for global constructions (e.g. CM points as in Section 4).

2.7. Lattices and Bruhat-Tits tree. We can phrase the description of automorphic forms on $B^{\times}$given in Section 2.6 in terms of the Bruhat-Tits tree. First consider the level $\Sigma\left(\mathfrak{a}, \mathfrak{n}^{-}\right)$with $\mathfrak{a}=\mathfrak{n}^{+}$. Let $\Phi \in S_{k}\left(\mathfrak{n}^{+}, \mathfrak{n}^{-}\right)$, corresponding to an $h$-tuple: $\left(\phi^{1}, \ldots, \phi^{h}\right)$ as in the previous section. For each $i=1, \ldots, h$, define a function $c_{\phi^{i}}$ on the set of all lattices of $F_{\mathfrak{p}} \oplus F_{\mathfrak{p}}$ as follows: given a lattice $L$, let $g_{L} \in \operatorname{GL}_{2}\left(F_{\mathfrak{p}}\right)$ be such that $L=g_{L}\left(\mathcal{O}_{F_{\mathfrak{p}}} \oplus \mathcal{O}_{F_{\mathfrak{p}}}\right)$, define

$$
c_{\phi^{i}}(L)=g_{L} \cdot \phi^{i}\left(g_{L}\right) .
$$

By (2.15), this is well-defined independent of the choice of $g_{L}$. It also follows from (2.15) the following property:

$$
c_{\phi^{i}}(\gamma L)=\gamma \cdot c_{\phi^{i}}(L) \text { for } \gamma \in \widetilde{\Gamma}_{i}\left(\mathfrak{n}^{+}, \mathfrak{n}^{-}\right) .
$$


In particular,

$$
c_{\phi^{i}}(p L)=p^{d(k-2)} c_{\phi^{i}}(L)=q^{k-2} c_{\phi^{i}}(L) .
$$

Similarly, suppose we take $\mathfrak{a}=\mathfrak{p} \mathfrak{n}^{+}$. If $\Phi \in S_{k}\left(\mathfrak{p} \mathfrak{n}^{+}, \mathfrak{n}^{-}\right)$, then corresponding to the $h$-tuple $\left(\phi^{1}, \ldots, \phi^{h}\right)$, we can define functions $\left(c_{1}, \ldots, c_{h}\right)$, where $c_{\phi^{i}}$ is a function on pairs of lattices

$$
L_{2} \subset L_{1} \text { of index equal to } q
$$

by the rule

$$
c_{\phi^{i}}\left(L_{1}, L_{2}\right)=g_{L} \cdot \phi^{i}\left(g_{L}\right)
$$

with $g_{L} \in \mathrm{GL}_{2}\left(F_{\mathfrak{p}}\right)$, such that $g_{L}\left(\mathcal{O}_{F_{\mathfrak{p}}} \oplus \mathcal{O}_{F_{\mathfrak{p}}}\right)=L_{1}, g_{L}\left(\mathcal{O}_{F_{\mathfrak{p}}} \oplus p \mathcal{O}_{F_{\mathfrak{p}}}\right)=L_{2}$.

We have

$$
\begin{gathered}
c_{\phi^{i}}\left(\gamma L_{1}, \gamma L_{2}\right)=\gamma \cdot c_{\phi^{i}}\left(L_{1}, L_{2}\right) \quad \text { for all } \gamma \in \widetilde{\Gamma}_{i}\left(\mathfrak{p} \mathfrak{n}^{+}, \mathfrak{n}^{-}\right), \\
c_{\phi^{i}}\left(p L_{1}, p L_{2}\right)=q^{k-2} c_{\phi^{i}}\left(L_{1}, L_{2}\right) .
\end{gathered}
$$

In both cases, we denote by $c_{\Phi}$ the vector of functions $\left\{c_{\phi^{i}}\right\}_{i=1}^{h}$.

When $k=2$, the functions $c_{\phi^{i}}(L), c_{\phi^{i}}\left(L_{1}, L_{2}\right)$ factor through homothety classes of lattices and pairs of lattices respectively, hence correspond to functions on the set of vertices $\mathcal{T}_{0}$, respectively edges $\mathcal{E}(\mathcal{T})$, of the Bruhat-Tits tree $\mathcal{T}$ of $\mathrm{PGL}_{2}\left(F_{\mathfrak{p}}\right)$ (by an edge it is always understood to be oriented).

The set of even (resp. odd) vertices of the $\mathcal{T}$, noted as $\mathcal{T}_{0}^{+}$(resp. $\mathcal{T}_{0}^{-}$), is the set of vertices whose distances to the class of the lattice $\mathcal{O}_{F_{\mathfrak{p}}} \oplus \mathcal{O}_{F_{\mathfrak{p}}}$ is an even (resp. odd) integer. An edge will be called even (resp. odd) if its origin is even (resp. odd), and we denote by $\mathscr{E}(\mathcal{T})^{+}$the set of even edges (resp. $\mathscr{E}(\mathcal{T})^{-}$for the set of odd edges).

On forms of weight two, the Hecke operators $T_{\mathfrak{p}}, U_{\mathfrak{p}}$ has the following interpretation:

$$
\begin{aligned}
c_{T_{\mathfrak{p}} \Phi}(L) & =\sum_{\tilde{L} \subset L} c_{\Phi}(\widetilde{L}), \\
c_{U_{\mathfrak{p}} \Phi}\left(L_{1}, L_{2}\right) & =\sum_{\tilde{L} \subset L_{2}} c_{\Phi}\left(L_{2}, \tilde{L}\right),
\end{aligned}
$$

where the first sum runs over the $q+1$ sub-lattices $\widetilde{L}$ of $L$ of index equal to $q$. The second sum runs over the sub-lattices $\widetilde{L}$ of $L_{2}$ of index equal to $q$, other than $p L_{1}$.

Suppose that $\Phi^{\#} \in S_{k}\left(\mathfrak{n}^{+}, \mathfrak{n}^{-}\right)$is an eigenvector for the Hecke operator $T_{\mathfrak{p}}$, with eigenvalue $C\left(\mathfrak{p}, \Phi^{\#}\right)$. Assume that $\Phi^{\#}$ is ordinary at $\mathfrak{p}$ (i.e., $C\left(\mathfrak{p}, \Phi^{\#}\right)$ is a $p$-adic unit). Let $\alpha\left(\mathfrak{p}, \Phi^{\#}\right)$ be the unit root of the characteristic polynomial

$$
x^{2}-C\left(\mathfrak{p}, \Phi^{\#}\right) x+q^{k-1} .
$$


Then we can define $\Phi \in S_{k}\left(\mathfrak{p} \mathfrak{n}^{+}, \mathfrak{n}^{-}\right)$to be the form whose associated function $c_{\Phi}$ satisfies

$$
c_{\Phi}\left(L_{1}, L_{2}\right)=c_{\Phi^{\#}}\left(L_{2}\right)-\frac{q^{k-2}}{\alpha\left(\mathfrak{p}, \Phi^{\#}\right)} c_{\Phi^{\#}}\left(L_{1}\right),
$$

which is an eigenvector for $U_{\mathfrak{p}}$ with eigenvalue $\alpha\left(\mathfrak{p}, \Phi^{\#}\right)$, as is easily verified. We call $\Phi$ the $\mathfrak{p}$-stabilization of $\Phi^{\#}$. It is easy to check that $\operatorname{Tr}_{\mathfrak{n}^{+}}^{\mathfrak{p} \mathfrak{n}^{+}} \Phi=\left(\alpha-\frac{q^{k-2}}{\alpha}\right) \Phi^{\#}$ (where $\alpha=\alpha\left(\mathfrak{p}, \Phi^{\#}\right)$ ). Hence $\Phi$ is old at $\mathfrak{p}$ (in the sense of Section 2.3).

We now come back to the setting as in the introduction, namely we take a cuspidal Hilbert eigenform $\mathbf{f}$ of parallel weight two, with conductor $\mathfrak{p n}^{+} \mathfrak{n}^{-}$. Since $\mathbf{f}$ is supposed to correspond to an elliptic curve $E / F$, we assume that the normalized Fourier coefficients $C(\mathfrak{m}, \mathbf{f})$ are all (rational) integers. As $\mathbf{f}$ is new at all prime dividing $\mathfrak{n}^{-}$, it corresponds under the Jacquet-Langlands' correspondence to an eigenform $\Phi \in S_{2}\left(\mathfrak{p} \mathfrak{n}^{+}, \mathfrak{n}^{-}\right)$, which is unique up to scalar multiples in $\mathbb{C}_{p}^{\times}$. Since the Hecke eigenvalues of $\Phi$ are integers, we can actually choose $\Phi$ so that the values taken by $\Phi$ are integers.

As p exactly divides the conductor of $\mathbf{f}$, we have $C(\mathfrak{p}, \mathbf{f})^{2}=1$ ([36], Lemma 1.4.5). In particular $\mathbf{f}$ and hence $\Phi$ are ordinary at $\mathfrak{p}$. Now by Theorem 2.10 , we can lift the eigenform $\Phi=\Phi_{2}$ to an eigenform $\Phi_{\infty} \in S\left(\Sigma\left(\mathfrak{n}^{+}, \mathfrak{n}^{-}\right)\right.$, $\left.D_{*}^{\text {cycl, } \dagger}\right)$, which corresponds to the Hida family $\mathbf{f}_{\infty}$ of cuspidal Hilbert eigenforms lifting $\mathbf{f}=\mathbf{f}_{2}$. If $U$ is a $p$-adic disk around two such that the weight $k$ specialization maps are defined for $k \in U \cap \mathbb{Z}^{\geq 2}$, then $\Phi_{k}$ corresponds to $\mathbf{f}_{k}$ under the Jacquet-Langlands' correspondence (the conductor of each $\mathbf{f}_{k}$ divides $\mathfrak{p} \mathfrak{n}^{+} \mathfrak{n}^{-}$and is divisible by $\mathfrak{n}^{+} \mathfrak{n}^{-}$). We only need to look at the specializations $\Phi_{k}$ and $\mathbf{f}_{k}$, with $k \equiv 2 \bmod p-1$, in which case $\Phi_{k} \in S_{k}\left(\mathfrak{p} \mathfrak{n}^{+}, \mathfrak{n}^{-}\right)$.

Now since the forms $\mathbf{f}_{k}$ and $\Phi_{k}$ are ordinary at $\mathfrak{p}$, Lemma 1.4.5 of [36] implies that $\mathbf{f}_{k}$ and hence $\Phi_{k}$ cannot be new at $\mathfrak{p}$, when $k>2, k \equiv 2 \bmod p-1$. For $k>2$, $k \equiv 2 \bmod p-1$, denote by $\mathbf{f}_{k}^{\#}$ the unique normalized cuspidal Hilbert eigenform of parallel weight $k$, conductor $\mathfrak{n}^{+} \mathfrak{n}^{-}$, such that $\mathbf{f}_{k}$ is the $\mathfrak{p}$-stabilization of $\mathbf{f}_{k}^{\#}$ (for the definition of $\mathfrak{p}$-stabilization of Hilbert modular forms see [26], equation (4.15)). Put $\Phi_{k}^{\#} \in S_{k}\left(\mathfrak{n}^{+}, \mathfrak{n}^{-}\right)$to be the unique eigenform on $B^{\times}$that corresponds to $\mathbf{f}_{k}^{\#}$ under the Jacquet-Langlands correspondence, and such that (2.18) is satisfied (although the Jacquet-Langlands correspondence determines $\Phi_{k}^{\#}$ only up to scalar multiples, equation (2.18) fixes the choice for $\Phi_{k}^{\#}$ uniquely). For notational consistency we put $\mathbf{f}_{2}^{\#}=\mathbf{f}_{2}$, and $\Phi_{2}^{\#}=\Phi_{2}$ (here we are using a different convention as compared to [4], where they put $\mathbf{f}_{2}^{\#}=0, \Phi_{2}^{\#}=0$ ). As in the situation of (2.17) and (2.18), for $k>2$ we have $C\left(\mathfrak{p}, \Phi_{k}\right)$, the $U_{\mathfrak{p}}$ eigenvalue of $\Phi_{k}$, is equal to $\alpha\left(\mathfrak{p}, \Phi_{k}^{\#}\right)$, and similarly $C\left(\mathfrak{p}, \mathbf{f}_{k}\right)=\alpha\left(\mathfrak{p}, \mathbf{f}_{k}^{\#}\right)$, where $\alpha\left(\mathfrak{p}, \mathbf{f}_{k}^{\#}\right)$ is the $p$-adic unit root of the Hecke polynomial $X^{2}-C\left(\mathfrak{p}, \mathbf{f}_{k}^{\#}\right) X+q^{k-1}\left(\right.$ for $\Phi_{2}$ and $\mathbf{f}_{2}$ we put $\alpha\left(\mathfrak{p}, \Phi_{2}^{\#}\right):=C\left(\mathfrak{p}, \Phi_{2}\right)$, $\left.\alpha\left(\mathfrak{p}, \mathbf{f}_{2}^{\#}\right):=C\left(\mathfrak{p}, \mathbf{f}_{2}\right)\right)$. 
We put $\alpha(\mathfrak{p}, k):=C\left(\mathfrak{p}, \Phi_{\infty}\right) \in \Lambda^{\dagger}$ (here $k \in U$ as a variable). Then we have $\alpha(\mathfrak{p}, k)=\alpha\left(\mathfrak{p}, \Phi_{k}^{\#}\right)=\alpha\left(\mathfrak{p}, \mathbf{f}_{k}^{\#}\right)$ for $k \in U \cap \mathbb{Z}^{\geq 2}, k \equiv 2 \bmod p-1$.

2.8. Some lemmas. We maintain the notations of sections 2.6 and 2.7. Thus let $\Phi_{\infty} \in S\left(\Sigma\left(\mathfrak{n}^{+}, \mathfrak{n}^{-}\right), \mathcal{D}_{*}^{\text {cycl, } \dagger}\right)$ be a measure-valued form, which corresponds, as in Section 2.6, to an $h$-tuple $\left(\phi_{\infty}^{1}, \ldots, \phi_{\infty}^{h}\right)$. Let $U$ be a $p$-adic neighbourhood of 2 such that for $k \in U \cap \mathbb{Z}^{\geq 2}$, the weight $k$ specializations $\Phi_{k}$ of $\Phi_{\infty}$ is defined. For $k \equiv 2$ $\bmod p-1$, we have $\Phi_{k} \in S_{k}\left(\mathfrak{p} \mathfrak{n}^{+}, \mathfrak{n}^{-}\right)$. If $k>2$, then $\Phi_{k}$ is the $\mathfrak{p}$-stabilization of $\Phi_{k}^{\#} \in S_{k}\left(\mathfrak{n}^{+}, \mathfrak{n}^{-}\right)$. Let $\left(\phi_{k}^{1}, \ldots, \phi_{k}^{h}\right)$ and $\left(\left(\phi_{k}^{\#}\right)^{1}, \ldots,\left(\phi_{k}^{\#}\right)^{h}\right)$ be the corresponding $h$-tuples of functions on $\mathrm{GL}_{2}\left(F_{\mathfrak{p}}\right)$ respectively.

Recall from Section 2.4 that $\mathscr{D}$ is the space of compactly supported $\mathbb{C}_{p}$-valued measures on $Y=c \backslash \mathcal{W}$. Put $\mathscr{D}^{\text {cycl }}=\mathscr{D} \otimes_{\tilde{\Lambda}_{F}} \widetilde{\Lambda}_{\mathbb{Q}}$ and $\mathscr{D}^{\text {cycl, } \dagger}=\mathscr{D}^{\text {cycl }} \otimes_{\Lambda} \hat{\otimes} \mathcal{O}_{\mathbb{C}_{p}} \Lambda^{\dagger}$, so that the injection $s: \mathscr{D}_{*} \hookrightarrow \mathscr{D}$ (extension by zero outside $X$ ) induces the injection $s: \mathscr{D}_{*}^{\text {cycl, } \dagger} \hookrightarrow \mathscr{D}^{\text {cycl, }, \dagger}$. We identify $\mathcal{D}_{*}^{\text {cycl, } \dagger}$ with its image in $\mathscr{D}^{\text {cycl, } \dagger}$ via the map $s$. Elements of $\mathscr{D}^{\text {cycl, } \dagger}$ can be used to integrate functions on $Y$ that are homogeneous of weight $\left\langle\chi_{F \text {,cycl }}\right\rangle^{k-2}$ for $k \in U$.

Recall that we have an action of $\mathrm{GL}_{2}\left(F_{\mathfrak{p}}\right)$ on $\mathcal{D}$ as in $(2.8)$. Extend it to an action on $\mathscr{D}^{\text {cycl, } \dagger}$ in the natural way (i.e., trivial action on the factor $\Lambda^{\dagger}$ ).

Definition 2.11. For a lattice $L$ of $F_{\mathfrak{p}} \oplus F_{\mathfrak{p}}$ and $i \in 1, \ldots, h$, define the element $\mu_{i, L} \in \mathscr{D}^{\mathrm{cycl}, \dagger}$ by

$$
\mu_{i, L}=g_{L} \cdot \phi_{\infty}^{i}\left(g_{L}\right),
$$

where $g_{L} \in \operatorname{GL}_{2}\left(F_{\mathfrak{p}}\right)$ satisfies $L=g_{L}\left(\mathcal{O}_{F_{\mathfrak{p}}} \oplus \mathcal{O}_{F_{\mathfrak{p}}}\right)$.

For any compact open subset $S$ of $Y$, and homogeneous function $F$ on $Y$ of weight $\left\langle\chi_{F, \text { cycl }}\right\rangle^{k-2}$ with $k \in U$, we have

$$
\int_{\mathcal{S}} F d \mu_{i, L}=\int_{g_{L}^{-1} \mathcal{S}} F \mid g_{L} d \phi_{\infty}^{i}\left(g_{L}\right) .
$$

It follows that the measure $\mu_{i, L}$ is supported on $c \backslash g_{L}\left(\left(\mathcal{O}_{F_{\mathfrak{p}}}^{2}\right)^{\prime}\right)=\mathfrak{c} \backslash L^{\prime}$, where $L^{\prime}=\{l \in L, l \notin p L\}$, the set of primitive vectors of $L$.

We list several lemmas, whose proofs follow exactly as in Section 2.4 of [4], so we just give the statements.

Lemma 2.12. For any $\gamma \in \widetilde{\Gamma}_{i}\left(\mathfrak{n}^{+}, \mathfrak{n}^{-}\right)$, and $F$ a homogeneous function of weight $\left\langle\chi_{F, \text { cycl }}\right\rangle^{k-2}$, with $k \in U$, we have, for $S$ an open compact subset of $Y$, that

$$
\int_{\gamma \mathcal{S}}\left(F \mid \gamma^{-1}\right) d \mu_{i, \gamma L}=\int_{\mathcal{S}} F d \mu_{i, L} .
$$


Lemma 2.13. Let $L_{2} \subset L_{1}$ be a sublattice of index equal to $q$. Then for $k \in U \cap \mathbb{Z}^{\geq 2}$, $k \equiv 2 \bmod p-1$, and $F$ a homogeneous function of weight $\left\langle\chi_{F, \mathrm{cycl}}\right\rangle^{k-2}$,

$$
\int_{\mathfrak{c} \backslash L_{1}^{\prime} \cap L_{2}^{\prime}} F d \mu_{i, L_{2}}=\alpha\left(\mathfrak{p}, \Phi_{k}\right) \int_{\mathfrak{c} \backslash L_{1}^{\prime} \cap L_{2}^{\prime}} F d \mu_{i, L_{1}} .
$$

Lemma 2.14. With the notations of Lemma 2.13, we have, for $P \in \mathscr{B}_{k}$,

$$
\int_{\mathfrak{c} \backslash L_{1}^{\prime} \cap L_{2}^{\prime}} P d \mu_{i, L_{1}}=c_{\phi_{k}^{i}}\left(L_{1}, L_{2}\right)(P) .
$$

Proposition 2.15. With the above notations,

$$
\int_{\mathfrak{c} \backslash L^{\prime}} P d \mu_{i, L}=\alpha\left(\mathfrak{p}, \Phi_{k}\right)\left(1-\frac{q^{k-2}}{\alpha\left(\mathfrak{p}, \Phi_{k}\right)^{2}}\right) c_{\left(\phi_{k}^{*}\right)^{i}}(L)(P) .
$$

2.9. Periods of forms of weight two. Let $\Phi=\Phi_{2} \in S_{2}\left(\mathfrak{p} \mathfrak{n}^{+}, \mathfrak{n}^{-}\right)$be as in Section 2.7. Recall that we assume $\Phi$ is normalized so that it takes integer values, hence so are the functions $c_{\phi^{i}}$ defined by (2.16).

Let

$$
\operatorname{pr}: \mathfrak{c} \backslash \mathcal{W} \rightarrow \mathbf{P}^{1}\left(F_{\mathfrak{p}}\right)
$$

be the natural projection map sending $(x, y) \in \mathfrak{c} \backslash \mathcal{W}$ to $\frac{x}{y} \in \mathbf{P}^{1}\left(F_{\mathfrak{p}}\right)$. For an even lattice $L$ define, for $i=1, \ldots, h$,

$$
\mu_{\phi^{i}}=\operatorname{pr}_{*}\left(\mu_{i, L}\right),
$$

i.e., for any open compact $y \subset \mathbf{P}^{1}\left(F_{\mathfrak{p}}\right)$, and continuous function $h$ on $\mathbf{P}^{1}\left(F_{\mathfrak{p}}\right)$,

$$
\int_{y} h d \mu_{\phi^{i}}=\int_{\mathrm{pr}^{-1}(y) \cap\left(c \backslash L^{\prime}\right)} h \circ \operatorname{pr} d \mu_{i, L}
$$

(note that $\mu_{i, L}$ is supported on $\mathfrak{c} \backslash L^{\prime}$ ).

Lemma 2.16 ([4], Lemma 2.12). The measures $\mu_{\phi^{i}}$ do not depend on the choice of the even lattice $L$.

Definition 2.17. Put $\mathrm{GL}_{2}^{+}\left(F_{\mathfrak{p}}\right):=\left\{g \in \mathrm{GL}_{2}\left(F_{\mathfrak{p}}\right), \operatorname{ord}_{p} \operatorname{det}(\gamma) \equiv 0 \bmod 2\right\}$.

The group $\mathrm{GL}_{2}^{+}\left(F_{\mathfrak{p}}\right)$ preserves the set of even lattices. Also note that if $\gamma \in$ $\tilde{\Gamma}_{i} \cap \mathrm{GL}_{2}^{+}\left(F_{\mathfrak{p}}\right)$, then $\operatorname{Nrd}_{B / F}(\gamma)=p^{2 r} u$ for some integer $r$ and $u \in \mathcal{O}_{F,+}^{\times}$, which implies that $p^{-r} \gamma \in \Gamma_{i}$. 
Lemma 2.18 ([4], Corollary 2.14). For $i=1, \ldots, h$, the measures $\mu_{\phi^{i}}$ are invariant under $\Gamma_{i}$, and satisfy

$$
\mu_{\phi^{i}}\left(\mathbf{P}^{1}\left(F_{\mathfrak{p}}\right)\right)=0 .
$$

Let $\mathscr{H}=\mathscr{H}_{/ F_{\mathfrak{p}}}$ be the rigid analytic $p$-adic upper half plane defined over $F_{\mathfrak{p}}$. The set of $\mathbb{C}_{p}$-points of $\mathcal{H}$ is given by $\mathcal{H}\left(\mathbb{C}_{p}\right)=\mathbf{P}^{1}\left(\mathbb{C}_{p}\right)-\mathbf{P}^{1}\left(F_{\mathfrak{p}}\right)=\mathbb{C}_{p}-F_{\mathfrak{p}}$. It is equipped with the Möbius action of $\mathrm{GL}_{2}\left(F_{\mathfrak{p}}\right)$ :

$$
\left(\begin{array}{ll}
a & b \\
c & d
\end{array}\right) \cdot z=\frac{a z+b}{c z+d} .
$$

Associated to the measures $\mu_{\phi^{i}}$ are the rigid analytic functions $f_{\phi^{i}}(z)$ on $\mathscr{H}\left(\mathbb{C}_{p}\right)$ :

$$
f_{\phi^{i}}(z)=\int_{\mathbf{P}^{1}\left(F_{\mathfrak{p}}\right)} \frac{1}{t-z} d \mu_{\phi^{i}}(t) .
$$

A direct calculation shows for any $\gamma \in \Gamma_{i}$, with $\left(\begin{array}{ll}a & b \\ c & d\end{array}\right)$ being the image of $\gamma$ in $B_{\mathfrak{p}}^{\times} \cong \mathrm{GL}_{2}\left(F_{\mathfrak{p}}\right)$, we have

$$
f_{\phi^{i}}(\gamma z)=\operatorname{Nrd}_{B / F}(\gamma)^{-1}(c z+d)^{2} f_{\phi^{i}}(z) .
$$

Put $\omega_{\phi^{i}}:=f_{\phi^{i}}(z) d z$. Then $\omega_{\phi^{i}}$ is invariant under the action of $\Gamma_{i}$, hence defines a rigid analytic differential form on the quotient $\Gamma_{i} \backslash \mathscr{H}$.

For $\tau_{1}, \tau_{2} \in \mathscr{H}\left(\mathbb{C}_{p}\right)$, define the period integral of Coleman:

$$
\int_{\tau_{1}}^{\tau_{2}} \omega_{\phi^{i}}:=\int_{\mathbf{P}^{1}\left(F_{\mathfrak{p}}\right)} \log _{p}\left(\frac{t-\tau_{2}}{t-\tau_{1}}\right) d \mu_{\phi^{i}}(t),
$$

where $\log _{p}$ is Iwasawa's $p$-adic logarithm, satisfying $\log _{p}(p)=0$. The following relations hold:

$$
\begin{gathered}
\int_{\tau_{1}}^{\tau_{2}} \omega_{\phi^{i}}+\int_{\tau_{2}}^{\tau_{3}} \omega_{\phi^{i}}=\int_{\tau_{1}}^{\tau_{3}} \omega_{\phi^{i}}, \\
\int_{\gamma \tau_{1}}^{\gamma \tau_{2}} \omega_{\phi^{i}}=\int_{\tau_{1}}^{\tau_{2}} \omega_{\phi^{i}} \quad \text { for } \gamma \in \Gamma_{i} .
\end{gathered}
$$

Under our assumption on the normalization of $\Phi$, the measure $\mu_{\phi^{i}}$ is $\mathbb{Z}$-valued, and we can define the multiplicative integral (for the precise definition see [12], equation (5.8)):

$$
\oiint_{\tau_{1}}^{\tau_{2}} \omega_{\phi^{i}}:=\oiint_{\mathbf{P}^{1}\left(F_{\mathfrak{p}}\right)}\left(\frac{t-\tau_{2}}{t-\tau_{1}}\right) d \mu_{\phi^{i}}(t) .
$$

It satisfies the multiplicative analogue of (2.20) and (2.21). We also have the formula

$$
\int_{\tau_{1}}^{\tau_{2}} \omega_{\phi^{i}}=\log _{p} \S_{\tau_{1}}^{\tau_{2}} \omega_{\phi^{i}}
$$


2.10. Periods and Hida families. Let $\mathbb{Q}_{p^{2 d}}$ be the unramified quadratic extension of $F_{\mathfrak{p}}=\mathbb{Q}_{p^{d}}$. For $\tau \in \mathbb{Q}_{p^{2 d}}$, let $\bar{\tau}$ be the image of $\tau$ under the non-trivial element of $\operatorname{Gal}\left(\mathbb{Q}_{p^{2 d}} / \mathbb{Q}_{p^{d}}\right)$.

For $\tau \in \mathscr{H}\left(\mathbb{Q}_{p^{2 d}}\right)=\mathbb{Q}_{p^{2 d}}-F_{\mathfrak{p}}$, define

$$
L_{\tau}:=\left\{(x, y) \in F_{\mathfrak{p}}^{2}, \operatorname{ord}_{p}(x-\tau y) \geq 0\right\} .
$$

It is seen that $L_{\tau}$ is a lattice of $F_{\mathfrak{p}} \oplus F_{\mathfrak{p}}$. The reduction map from $\mathscr{H}\left(\mathbb{Q}_{p}^{\text {ur }}\right)$ to $\mathcal{T}_{0}$ (where $\mathbb{Q}_{p}^{\text {ur }}$ is the maximal unramified extension of $\mathbb{Q}_{p}$ ) sends $\tau$ to the homothety class of $L_{\tau}$ (see [14], Section 1.3). Denote by $v_{\tau} \in \mathcal{T}_{0}$ the class of $L_{\tau}$.

By direct calculation, for any $\gamma=\left(\begin{array}{ll}a & b \\ c & d\end{array}\right) \in \operatorname{GL}_{2}\left(F_{\mathfrak{p}}\right)$,

$$
L_{\gamma \tau}=p^{t} \gamma L_{\tau}
$$

where $t=\operatorname{ord}_{p}\left((\operatorname{det} \gamma)^{-1}(c \tau+d)\right)$, which implies

$$
v_{\gamma \tau}=\gamma v_{\tau}
$$

for any $\gamma \in \mathrm{GL}_{2}\left(F_{\mathfrak{p}}\right)$.

An element $\tau \in \mathscr{H}\left(\mathbb{Q}_{p^{2 d}}\right)$ is said to be even (resp. odd) if $v_{\tau}$ is even (resp. odd). The set of even (resp. odd) elements is preserved by $\mathrm{GL}_{2}^{+}\left(F_{\mathfrak{p}}\right)$.

For any $x \in\left(\mathbb{Q}_{p}^{\text {ur }}\right)^{\times}$, write $\langle x\rangle$ for the projection of $x$ to the one-units, i.e.,

$$
x=p^{\operatorname{ord} p(x)} \zeta_{x}\langle x\rangle
$$

with $\zeta_{x}$ a root of unity of order prime to $p$. We make a definition.

Definition 2.19. Let $F$ be a homogeneous function on $\mathfrak{c} \backslash \mathcal{W}$ of weight $\left\langle\chi_{F \text {,cycl }}\right\rangle^{s}$ for some $s \in \mathbb{Z}_{p}-\{0\}$, and $S \subset \mathfrak{c} \backslash \mathcal{W}$ an open compact subset. Assume that $F$ is non-zero on $\delta$. Define

$$
\int_{\mathcal{S}} \log _{p} F(x, y) d \mu_{i, L_{\tau}}(x, y):=\left.s \frac{d}{d k}\left(\int_{\mathcal{S}}\langle F(x, y)\rangle^{s^{-1}(k-2)} d \mu_{i, L_{\tau}}(x, y)\right)\right|_{k=2},
$$

where $k$ takes values in a $p$-adic neighborhood of 2 . It is not hard to verify that the derivative exists.

Definition 2.20. For even $\tau \in \mathscr{H}\left(\mathbb{Q}_{p^{2 d}}\right)$, define the function $F_{\tau}$ on $c \backslash \mathcal{W}$ by

$$
F_{\tau}(x, y):=\left\langle\mathcal{N}_{\mathbb{Q}_{p^{2 d}} / \mathbb{Q}_{p}}(x-\tau y)\right\rangle .
$$

The function $F_{\tau}$ is homogeneous of weight $\left\langle\chi_{F \text {,cycl }}\right\rangle^{2}$. Note that we can also write

$$
F_{\tau}(x, y)=\left\langle\mathcal{N}_{F_{\mathfrak{p}} / \mathbb{Q}_{p}}((x-\tau y)(x-\bar{\tau} y))\right\rangle .
$$


It is clear that $F_{\tau}$ is nonzero on $\mathrm{c} \backslash L_{\tau}^{\prime}$.

Define

$$
\begin{aligned}
I_{\phi^{i}}(\tau) & :=\int_{\mathfrak{c} \backslash L_{\tau}^{\prime}} \log _{p} F_{\tau}(x, y) d \mu_{i, L_{\tau}}(x, y) \\
& =\left.2 \frac{d}{d k}\left(\int_{\mathfrak{c} \backslash L_{\tau}^{\prime}}\langle F(x, y)\rangle^{\frac{k-2}{2}} d \mu_{i, L_{\tau}}(x, y)\right)\right|_{k=2} .
\end{aligned}
$$

Proposition 2.21. For any $\gamma \in \widetilde{\Gamma}_{i}$ we have

$$
I_{\phi^{i}}(\gamma \tau)=I_{\phi^{i}}(\tau)
$$

Proof. The function $\left\langle F_{\tau}(x, y)\right\rangle^{\frac{k-2}{2}}$ is homogeneous of weight $\left\langle\chi_{F \text {,cycl }}\right\rangle^{k-2}$. For $\gamma=\left(\begin{array}{ll}a & b \\ c & d\end{array}\right)$ we have, with the notations of equation (2.22),

$$
\begin{aligned}
\int_{\mathfrak{c} \backslash L_{\gamma \tau}^{\prime}} & \left\langle F_{\gamma \tau}(x, y)\right\rangle^{\frac{k-2}{2}} d \mu_{i, L_{\gamma \tau}}(x, y) \\
= & \int_{\mathfrak{c} \backslash p^{t} \gamma L_{\tau}^{\prime}}\left\langle F_{\gamma \tau}(x, y)\right\rangle^{\frac{k-2}{2}} d \mu_{i, p^{t} \gamma L_{\tau}}(x, y) \\
= & \int_{\mathfrak{c} \backslash L_{\tau}^{\prime}}\left\langle\left(F_{\gamma \tau} \mid p^{t} \gamma\right)(x, y)\right\rangle^{\frac{k-2}{2}} d \mu_{i, L_{\tau}}(x, y) \quad \text { (by Lemma 2.12). }
\end{aligned}
$$

Now by direct computation

$$
\left(F_{\gamma \tau} \mid p^{t} \gamma\right)(x, y)=\left\langle\mathcal{N}_{\mathbb{Q}_{p^{2 d}} / \mathbb{Q}_{p}}\left(\frac{\operatorname{det} \gamma}{c \tau+d}\right)\right\rangle \cdot F_{\tau}(x, y) .
$$

Hence

$$
\begin{aligned}
\int_{\mathfrak{c} \backslash L_{\gamma \tau}^{\prime}}\left\langle F_{\gamma \tau}(x, y)\right\rangle^{\frac{k-2}{2}} d \mu_{i, L_{\gamma \tau}}(x, y) \\
=\left\langle\mathcal{N}_{\mathbb{Q}_{p} 2 d} / \mathbb{Q}_{p}\left(\frac{\operatorname{det} \gamma}{c \tau+d}\right)\right\rangle^{\frac{k-2}{2}} \int_{\mathfrak{c} \backslash L_{\tau}^{\prime}}\left\langle F_{\tau}(x, y)\right\rangle^{\frac{k-2}{2}} d \mu_{i, L_{\tau}}(x, y) .
\end{aligned}
$$

Taking derivative at $k=2$ and applying Lemma 2.18, the result follows.

Thus the value of $I_{\phi^{i}}(\tau)$ depends only on the image of $\tau$ modulo $\widetilde{\Gamma}_{i}$. By multiplying $\tau$ with an element of $\gamma \in \widetilde{\Gamma}_{i}$ with $\operatorname{Nrd}_{B / F}(\gamma)=p$ if necessary (that such an element exists is seen by using Corollary 5.9 of [34], Chapter 3), one can always normalize $\tau$ to be even.

Let $\mathscr{H}^{\prime}\left(\mathbb{Q}_{p^{2 d}}\right) \subset \mathscr{H}\left(\mathbb{Q}_{p^{2 d}}\right)$ be the set of even $\tau$ 's. It then follows that

$$
\Gamma_{i} \backslash \mathscr{H}^{\prime}\left(\mathbb{Q}_{p^{2 d}}\right) \cong \widetilde{\Gamma}_{i} \backslash \mathcal{H}\left(\mathbb{Q}_{p^{2 d}}\right) .
$$


Lemma 2.22. Let $L_{2}$ be a sublattice of $L_{1}$ of index equal to $q$, and let $e=$ $\left(\left[L_{1}\right],\left[L_{2}\right]\right) \in \mathcal{E}(\mathcal{T})$ denote the corresponding edge (going from $\left[L_{1}\right]$ to $\left.\left[L_{2}\right]\right)$. Then for any $\tau \in \mathscr{H}\left(\mathbb{Q}_{p^{2 d}}\right)$,

$$
\int_{\mathfrak{c} \backslash L_{1}^{\prime} \cap L_{2}^{\prime}} \log _{p} F_{\tau}(x, y) d\left(\mu_{i, L_{2}}-\mu_{i, L_{1}}\right)=\left.2 \frac{d}{d k} \alpha(\mathfrak{p}, k)\right|_{k=2} c_{\phi^{i}}(e)
$$

if $\alpha(p, \Phi)=1$, and

$$
\int_{\mathfrak{c} \backslash L_{1}^{\prime} \cap L_{2}^{\prime}} \log _{p} F_{\tau}(x, y) d\left(\mu_{i, L_{2}}+\mu_{i, L_{1}}\right)=\left.2 \frac{d}{d k} \alpha(\mathfrak{p}, k)\right|_{k=2} c_{\phi^{i}}(e)
$$

if $\alpha(p, \Phi)=-1$.

Proof. For $k \in U \cap \mathbb{Z}^{\geq 2}, k \equiv 2 \bmod p-1$, we have by Lemma 2.13,

$$
\begin{aligned}
& \int_{\mathfrak{c} \backslash L_{1}^{\prime} \cap L_{2}^{\prime}}\left\langle F_{\tau}(x, y)\right\rangle^{\frac{k-2}{2}} d\left(\mu_{i, L_{2}} \pm \mu_{i, L_{1}}\right) \\
& \quad=(\alpha(\mathfrak{p}, k) \pm 1) \int_{\mathfrak{c} \backslash L_{1}^{\prime} \cap L_{2}^{\prime}}\left\langle F_{\tau}(x, y)\right\rangle^{\frac{k-2}{2}} d \mu_{i, L_{1}} .
\end{aligned}
$$

The result follows from taking derivatives, and using Lemma 2.14 which gives

$$
\int_{\mathfrak{c} \backslash L_{1}^{\prime} \cap L_{2}^{\prime}} 1 d \mu_{i, L_{1}}=c_{\phi^{i}}(e) .
$$

For an edge $e$ of $\mathcal{T}$, define $|e|$ to be 0 resp. 1, if $e$ is even, resp. odd.

Lemma 2.23. Let $L_{1}, L_{2}$ be even lattices of $F_{\mathfrak{p}} \oplus F_{\mathfrak{p}}$, and $v_{1}, v_{2}$ be the corresponding vertices of $\mathcal{T}$. Then

$$
\begin{aligned}
& \int_{\mathfrak{c} \backslash L_{1}^{\prime} \cup L_{2}^{\prime}} \log _{p} F_{\tau}(x, y) d\left(\mu_{i, L_{2}}-\mu_{i, L_{1}}\right) \\
& =\left.4 \frac{d}{d k} \alpha(\mathfrak{p}, k)\right|_{k=2} \cdot \sum_{e: v_{1} \rightarrow v_{2}} \alpha_{\mathfrak{p}}^{|e|} c_{\phi^{i}}(e),
\end{aligned}
$$

where the sum is over the oriented edges $e$ in the path that goes from $v_{1}$ to $v_{2}$, and $\alpha_{\mathfrak{p}}=\alpha(\mathfrak{p}, \Phi)$. If $\alpha(\mathfrak{p}, \Phi)=1$, then (2.24) holds without the parity condition on the lattices.

Proof. This follows easily from Lemma 2.22, cf. the proof of Lemma 2.23 of [4]. 
Proposition 2.24. For even $\tau_{1}, \tau_{2} \in \mathscr{H}^{\prime}\left(\mathbb{Q}_{p^{2 d}}\right)$,

$$
\begin{aligned}
& I_{\phi^{i}}\left(\tau_{2}\right)-I_{\phi^{i}}\left(\tau_{1}\right) \\
& \quad=\log _{p} \mathcal{N}_{\mathbb{Q}_{p^{2 d}} / \mathbb{Q}_{p}}\left(\oiint_{\tau_{1}}^{\tau_{2}} \omega_{\phi^{i}}\right)+\left.4 \frac{d}{d k} \alpha(\mathfrak{p}, k)\right|_{k=2} \cdot \operatorname{val}_{\mathfrak{p}}\left(\oiint_{\tau_{1}}^{\tau_{2}} \omega_{\phi^{i}}\right) .
\end{aligned}
$$

If $\alpha(\mathfrak{p}, \Phi)=1$, then the equality holds without the parity condition on $\tau_{1}, \tau_{2}$.

Proof. Set $L_{j}=L_{\tau_{j}}$. We have

$$
\begin{aligned}
I_{\phi^{i}}\left(\tau_{2}\right)-I_{\phi^{i}}\left(\tau_{1}\right)= & \int_{\mathfrak{c} \backslash L_{1}^{\prime}}\left(\log _{p} F_{\tau_{2}}(x, y)-\log _{p} F_{\tau_{1}}(x, y)\right) d \mu_{i, L_{1}} \\
& +\int_{\mathfrak{c} \backslash L_{1}^{\prime} \cup L_{2}^{\prime}} \log _{p} F_{\tau_{2}}(x, y) d\left(\mu_{i, L_{2}}-\mu_{i, L_{1}}\right) .
\end{aligned}
$$

The second term is handled by Lemma 2.23, together with the equality (which can be proved as in [4], Proposition 2.15)

$$
\operatorname{val}_{\mathfrak{p}}\left(f_{\tau_{1}}^{\tau_{2}} \omega_{\phi^{i}}\right)=\sum_{e: v_{1} \rightarrow v_{2}} \alpha_{\mathfrak{p}}^{|e|} c_{\phi^{i}}(e) .
$$

For the first term:

$$
\begin{aligned}
\int_{\mathfrak{c} \backslash L_{1}^{\prime}} & \left(\log _{p} F_{\tau_{2}}(x, y)-\log _{p} F_{\tau_{1}}(x, y)\right) d \mu_{i, L_{1}} \\
= & \int_{\mathfrak{c} \backslash L_{1}^{\prime}} \log _{p} \frac{F_{\tau_{2}}}{F_{\tau_{1}}}(x, y) d \mu_{i, L_{1}} \\
= & \int_{\mathfrak{c} \backslash L_{1}^{\prime}} \log _{p} \mathcal{N}_{\mathbb{Q}_{p} 2 d} / \mathbb{Q}_{p}\left(\frac{x-\tau_{2} y}{x-\tau_{1} y}\right) d \mu_{i, L_{1}} \\
= & \int_{\mathbf{P}^{1}\left(F_{\mathfrak{p}}\right)} \log _{p} \mathcal{N}_{\mathbb{Q}_{p^{2 d}} / \mathbb{Q}_{p}}\left(\frac{x-\tau_{2} y}{x-\tau_{1} y}\right) d \mu_{\phi^{i}} \\
= & \log _{p} \mathcal{N}_{\mathbb{Q}_{p^{2 d}} / \mathbb{Q}_{p}} \oint_{\mathbf{P}^{1}\left(F_{\mathfrak{p}}\right)} \frac{x-\tau_{2} y}{x-\tau_{1} y} d \mu_{\phi^{i}} \\
= & \log _{p} \mathcal{N}_{\mathbb{Q}_{p^{2 d}} / \mathbb{Q}_{p}} \oint_{\tau_{1}}^{\tau_{2}} \omega_{\phi^{i}} .
\end{aligned}
$$

Hence the proof of the proposition.

2.11. The $\mathscr{L}$-invariant. We now assume, as in the introduction, that $E / F$ is an elliptic curve over $F$, with conductor $\mathfrak{n}$, with multiplicative reduction at $\mathfrak{p}$, and is modular, i.e., it corresponds to a cuspidal Hilbert newform $\mathbf{f}=\mathbf{f}_{E}$ of conductor $\mathfrak{n}$, 
parallel weight 2, such that $L(s, E / F)=L\left(s, \mathbf{f}_{E}\right)$. Assume as in Section 2.7 that $\mathfrak{n}$ can be written as $\mathfrak{n}=\mathfrak{p} \mathfrak{n}^{+} \mathfrak{n}^{-}$, with $\Phi=\Phi_{E}$ corresponds to $\mathbf{f}_{E}$ under the Jacquet-Langlands' correspondence (normalized to take integer values).

Since $E$ has multiplicative reduction at $\mathfrak{p}$, we have, by Tate's theory of $p$-adic uniformization, a parametrization $\Theta_{E / F_{\mathfrak{p}}}$ :

$$
\Theta_{E / F_{\mathfrak{p}}}: \mathbb{C}_{p}^{\times} / Q_{E}^{\mathbb{Z}} \stackrel{\sim}{\longrightarrow} E\left(\mathbb{C}_{p}\right),
$$

where $Q_{E} \in F_{\mathfrak{p}}=\mathbb{Q}_{p^{d}}$, with $\operatorname{ord}_{p} Q_{E}>0$, called the Tate period of $E$ at the prime $\mathfrak{p}$. The map $\Theta_{E / F_{\mathfrak{p}}}$ is defined over $\mathbb{Q}_{p^{2 d}}$, i.e., equivariant with respect to the action of $\operatorname{Gal}\left(\overline{\mathbb{Q}}_{p} / \mathbb{Q}_{p^{2 d}}\right)$ on both sides. Furthermore, if $s \in \operatorname{Gal}\left(\mathbb{Q}_{p^{2 d}} / \mathbb{Q}_{p^{d}}\right)$ is the nontrivial element, then for $z \in \mathbb{Q}_{p^{2 d}}^{\times}$,

$$
s\left(\Theta_{E / F_{\mathfrak{p}}}(z)\right)=\alpha_{\mathfrak{p}} \Theta_{E_{F_{\mathfrak{p}}}}(s \cdot z),
$$

where $\alpha_{\mathfrak{p}}=\alpha(\mathfrak{p}, E / F)=\alpha\left(\mathfrak{p}, \mathbf{f}_{E}\right)$. Thus in particular, if $\alpha_{\mathfrak{p}}=1$, i.e., $E / F$ is split multiplicative at $\mathfrak{p}$, then $\Theta_{E / F_{\mathfrak{p}}}$ is defined over $F_{\mathfrak{p}}$.

Let $E\left(\mathbb{Q}_{p^{2 d}}\right)_{\alpha_{\mathfrak{p}}} \subset E\left(\mathbb{Q}_{p^{2 d}}\right)$ be the subgroup consisting of points $P$ such that

$$
s \cdot P=\alpha_{\mathfrak{p}} P .
$$

Then it follows from $(2.25)$ that $E\left(\mathbb{Q}_{p^{2 d}}\right)_{\alpha_{\mathfrak{p}}}=\Theta_{E / F_{\mathfrak{p}}}\left(F_{\mathfrak{p}}\right)$.

Let $q_{E}:=\mathcal{N}_{F_{\mathfrak{p}} / \mathbb{Q}_{p}} Q_{E}$. The $\mathscr{L}$-invariant of $E$ at $\mathfrak{p}$ is defined as

$$
\mathscr{L}_{\mathfrak{p}}(E / F)=\frac{\log _{p} q_{E}}{\operatorname{val}_{\mathfrak{p}} Q_{E}}=f_{\mathfrak{p} / p} \frac{\log _{p} q_{E}}{\operatorname{ord}_{p} q_{E}} .
$$

(Here $f_{\mathfrak{p} / p}$ is the residue field degree of $F_{\mathfrak{p}}$ which, under our assumption that $p$ is inert in $F$, is equal to $\left[F_{\mathfrak{p}}: \mathbb{Q}_{p}\right]=[F: \mathbb{Q}]$.)

By [26], Proposition 8.7,

$$
\left.\frac{d}{d k} \alpha(\mathfrak{p}, k)\right|_{k=2}=-\frac{1}{2} \mathscr{L}_{\mathfrak{p}}(E / F) .
$$

In [26], this is proved by generalizing the argument of Greenberg-Stevens. Following Section 2.7 of [4], we can give another proof, based on the theory of $p$-adic uniformization of Shimura curves; for more details, see Section 4.5 below. For any $i \in\{1, \ldots, h\}$, there exists even $\tau \in \mathscr{H}^{\prime}\left(\mathbb{Q}_{p^{2 d}}\right), \gamma \in \Gamma_{i}$, and non-zero $n_{i} \in \mathbb{Z}$ such that

$$
\oiint_{\tau}^{\gamma \tau} \omega_{\phi^{i}}=Q_{E}^{n_{i}}
$$

Now Propositions 2.21 and 2.24 imply that

$$
\log _{p} \mathcal{N}_{\mathbb{Q}_{p^{2}} / \mathbb{Q}_{p}}\left(\oiint_{\tau}^{\gamma \tau} \omega_{\phi^{i}}\right)+\left.4 \frac{d}{d k} \alpha(\mathfrak{p}, k)\right|_{k=2} \cdot \operatorname{val}_{\mathfrak{p}}\left(\oiint_{\tau}^{\gamma \tau} \omega_{\phi^{i}}\right)=0 .
$$


Hence by equation (2.28), since $n_{i} \neq 0$,

$$
\log _{p} \mathcal{N}_{\mathbb{Q}_{p^{2 d}} / \mathbb{Q}_{p}} Q_{E}+\left.4 \frac{d}{d k} \alpha(\mathfrak{p}, k)\right|_{k=2} \cdot \operatorname{val}_{\mathfrak{p}} Q_{E}=0,
$$

so

$$
\begin{aligned}
\left.\frac{d}{d k} \alpha(\mathfrak{p}, k)\right|_{k=2} & =-\frac{1}{4} \frac{\log _{p} \mathcal{N}_{\mathbb{Q}_{p 2 d} / \mathbb{Q}_{p} Q_{E}}}{\operatorname{val}_{\mathfrak{p}} Q_{E}} \\
& =-\frac{1}{2} \frac{\log _{p} \mathcal{N}_{F_{\mathfrak{p}} / \mathbb{Q}_{p}} Q_{E}}{\operatorname{val}_{\mathfrak{p}} Q_{E}}=-\frac{1}{2} \mathscr{L}_{\mathfrak{p}}(E / F)
\end{aligned}
$$

and the claim follows.

For any $Q \in \overline{\mathbb{Q}}_{p}^{\times}$, let $\log _{Q}$ be the branch of the $p$-adic logarithm such that $\log _{Q}(Q)=0$, i.e.,

$$
\log _{Q}(\cdot)=\log _{p}(\cdot)-\frac{\log _{p} Q}{\operatorname{ord}_{p} Q} \operatorname{ord}_{p}(\cdot) .
$$

Using equation (2.26) and (2.27), we can restate Proposition 2.24 as follows:

Corollary 2.25. For even $\tau_{1}, \tau_{2}$,

$$
I_{\phi^{i}}\left(\tau_{2}\right)-I_{\phi^{i}}\left(\tau_{1}\right)=\log _{q_{E}} \mathcal{N}_{\mathbb{Q}_{p^{2 d}} / \mathbb{Q}_{p}}\left(\oiint_{\tau_{1}}^{\tau_{2}} \omega_{\phi^{i}}\right) .
$$

Definition 2.26. Given a divisor with rational coefficients $D \in \operatorname{Div}\left(\Gamma_{i} \backslash \mathscr{H}\left(\mathbb{C}_{p}\right)\right) \otimes \mathbb{Q}$ of the form $D=\sum_{\kappa} r_{\kappa} \tau_{\kappa}$, with $r_{\kappa} \in \mathbb{Q}, \tau_{\kappa} \in \Gamma_{i} \backslash \mathscr{H}\left(\mathbb{C}_{p}\right)$, define

$$
I_{\phi^{i}}(D)=\sum_{\kappa} r_{\kappa} I_{\phi^{i}}\left(\tau_{\kappa}\right)
$$

More generally let $D=\sum_{i=1}^{h} D_{i} \in \bigoplus_{i=1}^{h} \operatorname{Div}\left(\Gamma_{i} \backslash \mathscr{H}\left(\mathbb{C}_{p}\right)\right) \otimes \mathbb{Q}$. Define

$$
I_{\Phi}(D):=\sum_{i=1}^{h} I_{\phi^{i}}\left(D_{i}\right)
$$

Definition 2.27. Let $f \in \operatorname{Div}^{0}\left(\mathscr{H}^{\prime}\left(\mathbb{Q}_{p^{2 d}}\right)\right)$ be a divisor of degree zero, say written in the form

$$
f=\sum_{\kappa}\left(\tau_{\kappa}-\tilde{\tau}_{\kappa}\right)
$$

with $\tau_{\kappa}, \tilde{\tau}_{\kappa} \in \mathscr{H}^{\prime}\left(\mathbb{Q}_{p^{2 d}}\right)$. Define for $i=1, \ldots, h$,

$$
\oint_{f} \omega_{\phi^{i}}=\prod_{\kappa} \int_{\tilde{\tau}_{\kappa}}^{\tau_{\kappa}} \omega_{\phi^{i}}
$$


It is clear that this is well defined independently of the way $f$ is written in the form of (2.29). For $f=\sum_{i=1}^{h} f_{i} \in \bigoplus_{i=1}^{h} \operatorname{Div}^{0}\left(\mathscr{H}^{\prime}\left(\mathbb{Q}_{p^{2 d}}\right)\right)$, put

$$
f_{f} \omega_{\Phi}:=\prod_{i=1}^{h} f_{f_{i}} \omega_{\phi^{i}} .
$$

As a consequence of Corollary 2.25 and Proposition 2.21, we see that the quantity

$$
\log _{q_{E}} \mathcal{N}_{\mathbb{Q}_{p 2 d} / \mathbb{Q}_{p}}\left(f_{f} \omega_{\Phi}\right)
$$

depends only the image of $f$ in $\bigoplus_{i=1}^{h} \operatorname{Div}^{0}\left(\Gamma_{i} \backslash \mathcal{H}^{\prime}\left(\mathbb{Q}_{p^{2 d}}\right)\right)$. We can thus define, by linearity, the quantity

$$
\log _{q_{E}} \mathcal{N}_{\mathbb{Q}_{p 2 d} / \mathbb{Q}_{p}}\left(f_{D} \omega_{\Phi}\right)
$$

for $D \in \bigoplus_{i=1}^{h} \operatorname{Div}^{0}\left(\Gamma_{i} \backslash \mathscr{H}^{\prime}\left(\mathbb{Q}_{p^{2 d}}\right)\right) \otimes \mathbb{Q}$.

With these definitions, we can state the following form of Corollary 2.25:

Corollary 2.28. Let $D \in \operatorname{Div}^{0}\left(\Gamma_{i} \backslash \mathscr{H}^{\prime}\left(\mathbb{Q}_{p^{2 d}}\right)\right) \otimes \mathbb{Q}$. Then

$$
I_{\Phi}(D)=\log _{q_{E}} \mathcal{N}_{\mathbb{Q}_{p} 2 d} / \mathbb{Q}_{p}\left(\oiint_{D} \omega_{\Phi}\right) .
$$

For future reference, we record the following formula. For $\sigma \in \operatorname{Hom}\left(F, \overline{\mathbb{Q}}_{p}\right)=$ $\operatorname{Hom}\left(F_{\mathfrak{p}}, \overline{\mathbb{Q}}_{p}\right)$, let $E^{\sigma} / F_{\mathfrak{p}^{\sigma}}^{\sigma}$ be the elliptic curve obtained from $E / F_{\mathfrak{p}}$ by conjugating with $\sigma$. It is multiplicative at the prime $\mathfrak{p}^{\sigma}$ of $F^{\sigma}$ above $p$, whose Tate period is given by $Q_{E}^{\sigma}$.

Proposition 2.29. For any $z \in F_{\mathfrak{p}}^{\times}$, we have

$$
\log _{q_{E}}\left(\mathcal{N}_{F_{\mathfrak{p}} / \mathbb{Q}_{p}} z\right)=\sum_{\sigma \in \operatorname{Hom}\left(F, \overline{\mathbb{Q}}_{p}\right)} \log _{Q_{E}^{\sigma}} z^{\sigma} .
$$

Proof. This is a direct computation.

\section{Interpolation of special $L$-values along Hida family}

3.1. Rankin $L$-functions. In this section we recall the formalism of Rankin $L$ functions.

Let $K / F$ be a quadratic extension of $F$. A Hecke character $\psi_{K}$ of $K$ is said to be anti-cyclotomic, if $\psi_{K} \circ c=\psi_{K}^{-1}$ (here $c$ is the non-trivial element of $\operatorname{Gal}(K / F)$ ), 
and $\psi_{K}$ is trivial on the image of $\mathbf{A}_{F}^{\times}$in $\mathbf{A}_{K}^{\times}$. Given a cuspidal Hilbert newform $\mathbf{g}$ over $F$, and an anti-cyclotomic character $\psi_{K}$ over $K$, one defines the Rankin $L$-functions $L\left(s, \mathbf{g} / K, \psi_{K}\right)$ as in [41], [38].

In this paper, we consider the particular case of genus characters. An anticyclotomic character $\psi_{K}$ is called a genus character if it is unramified, i.e., factors through $\operatorname{Pic}\left(\mathcal{O}_{K}\right)$, and quadratic (namely of order dividing two). Genus characters can be constructed in the following manner.

Let $\zeta$ be the quadratic Hecke character of $F$, that corresponds, by class field theory, to the extension $K / F$. Consider quadratic Hecke characters $\psi_{1}, \psi_{2}$ of $F$, whose conductors are relatively prime to each other, and such that $\psi_{1} \cdot \psi_{2}=\zeta$.

It follows from class field theory, the Hecke characters $\psi_{i}$ correspond to characters of $\operatorname{Gal}(\bar{F} / F)$. Let $F^{\psi_{i}} / F$ be the quadratic extension of $F$ cut out by $\psi_{i}$, and let $F^{\psi_{1}, \psi_{2}}$ be the composite of $F^{\psi_{1}}$ and $F^{\psi_{2}}$ over $F$. Then $F^{\psi_{1}, \psi_{2}} / K$ is everywhere unramified (including the archimedean places) quadratic extension.

Let $t_{i} \in \operatorname{Gal}\left(F^{\psi_{i}} / F\right)$ be the nontrivial element. We have

$$
\operatorname{Gal}\left(F^{\psi_{1}, \psi_{2}} / F\right)=\operatorname{Gal}\left(F^{\psi_{1}} / F\right) \times \operatorname{Gal}\left(F^{\psi_{2}} / F\right),
$$

and under this decomposition $\operatorname{Gal}\left(F^{\left.\psi_{1}, \psi_{2} / K\right)}=\left\{(1,1),\left(t_{1}, t_{2}\right)\right\}\right.$. Let $\psi_{K}$ be the quadratic character of $\operatorname{Gal}(\bar{K} / K)$, that factors through $\operatorname{Gal}\left(F^{\psi_{1}, \psi_{2}} / K\right)$, and is equal to -1 on the nontrivial element of $\operatorname{Gal}\left(F^{\psi_{1}, \psi_{2}} / K\right)$. By class field theory, $\psi_{K}$ corresponds to a Hecke character of $K$, which can be seen to be a genus character. The bi-quadratic extension $F^{\psi_{1}, \psi_{2}} / F$ is called the genus field extension corresponding to the pair $\psi_{1}, \psi_{2}$. We have $F^{\psi_{1}, \psi_{2}}=K^{\psi_{K}}$ (the extension of $K$ cut out by $\psi_{K}$ ). If one of $\psi_{1}, \psi_{2}$ is trivial, then $F^{\psi_{1}, \psi_{2}}$ degenerates to $K$.

One has

$$
\operatorname{Ind}_{K}^{F} \psi_{K}=\psi_{1} \oplus \psi_{2}
$$

Using (3.1), we have the factorization formula for the Rankin $L$-functions associated to genus characters:

$$
L\left(s, \mathbf{g} / K, \psi_{K}\right)=L\left(s, \mathbf{g}, \psi_{1}\right) \cdot L\left(s, \mathbf{g}, \psi_{2}\right) .
$$

In the rest of the section $K / F$ is a $\mathrm{CM}$ extension.

3.2. Optimal embeddings. We maintain the notations of Section 2.3 , regarding the definite quaternion algebra $B$, and an Eichler order $R$ of level a.

Definition 3.1. An optimal embedding of $K$ into $B$ of level $a$ is a pair $(\Psi, b) \in$ $\operatorname{Hom}_{F}(K, B) \times \widehat{B}^{\times} / \widehat{R}^{\times}$satisfying

$$
\Psi\left(\mathcal{O}_{K}\right)=b \widehat{R} b^{-1} \cap \Psi(K) .
$$


Given an optimal embedding $(\Psi, b)$ of level $a$, define

$$
R_{b}:=B \cap b \widehat{R} b^{-1} \text {. }
$$

Then $R_{b}$ is an Eichler order of level $a$, and $\Psi$ gives an embedding of $\mathcal{O}_{K}$ into $R_{b}$.

Proposition 3.2 (Theorems 3.1 and 3.2 of [34]). Suppose that all primes dividing a splits in $K$, while those dividing $\mathrm{n}^{-}$are inert in $K$ (this is known as Heegner condition). Then optimal embeddings of level a exist.

The group $B^{\times}$acts on the set of optimal embeddings by conjugation:

$$
g \cdot(\Psi, b)=\left(g \Psi g^{-1}, g b\right) .
$$

We denote by $[\Psi, b]$ the conjugacy class containing $(\Psi, b)$. The set of conjugacy

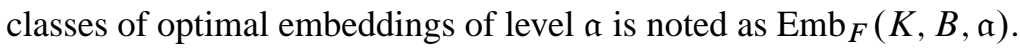

The ideal class group $\operatorname{Pic}\left(\mathcal{O}_{K}\right)$ acts simply transitively on the set of conjugacy classes of optimal embeddings: identify

$$
\operatorname{Pic}\left(\mathcal{O}_{K}\right)=\widehat{K}^{\times} / K^{\times} \widehat{\mathcal{O}}_{K}^{\times}
$$

then given $\rho \in \operatorname{Pic}\left(\mathcal{O}_{K}\right)$, we have

$$
\rho \cdot[\Psi, b]=\left[\Psi^{\rho}, b^{\rho}\right]:=[\Psi, \widehat{\Psi}(\rho) b] .
$$

Write $K=F(\lambda)$, with $\lambda^{2} \in F$ being totally negative. Given $\Psi \in \operatorname{Hom}_{F}(K, B)$, let $\left(\begin{array}{ll}a & b \\ c & d\end{array}\right)$ be the image of $\Psi(\lambda)$ in $B_{\mathfrak{p}} \stackrel{\mathfrak{l}_{\mathfrak{p}}}{\cong} \mathrm{GL}_{2}\left(F_{\mathfrak{p}}\right)$. Define $Q_{\Psi} \in \mathcal{B}_{4}$ by the following formula:

$$
\begin{gathered}
Q_{\Psi}=\frac{1}{\sqrt{\left|\mathcal{N}_{F / \mathbb{Q}}\left(\lambda^{2}\right)\right|}} \bigotimes_{\sigma \in \operatorname{Hom}\left(F_{\mathfrak{p}}, \overline{\mathbb{Q}}_{p}\right)} q_{\Psi, \sigma}, \text { where } \\
q_{\Psi, \sigma}\left(X^{\sigma}, Y^{\sigma}\right)=c^{\sigma}\left(X^{\sigma}\right)^{2}+\left(d^{\sigma}-a^{\sigma}\right) X^{\sigma} Y^{\sigma}-b^{\sigma}\left(Y^{\sigma}\right)^{2} .
\end{gathered}
$$

Up to the choice of sign for the square root, $Q_{\Psi}$ does not depend on the choice of $\lambda$. For $k \geq 2$ even we denote by $Q_{\Psi}^{\frac{k-2}{2}}$ the element of $\mathcal{B}_{k}$ obtained from $Q_{\Psi}$ in the evident manner, i.e.,

$$
Q_{\Psi}^{\frac{k-2}{2}}:=\left(\frac{1}{\sqrt{\left|\mathcal{N}_{F / \mathbb{Q}}\left(\lambda^{2}\right)\right|}}\right)^{\frac{k-2}{2}} \bigotimes_{\sigma \in \operatorname{Hom}\left(F_{\mathfrak{p}}, \overline{\mathbb{Q}}_{p}\right)} q_{\Psi, \sigma}^{\frac{k-2}{2}} .
$$

For $g \in B^{\times}$, we have

$$
Q_{g \Psi g^{-1}}=\mathcal{N}_{F / \mathbb{Q}}\left(\operatorname{Nrd}_{B / F}(g)\right) Q_{\Psi} \mid g_{\mathfrak{p}}^{-1} .
$$


Remark 3.3. Suppose that $\mathfrak{p}$ is inert in $K$, so $K_{\mathfrak{p}}=\mathbb{Q}_{p^{2 d}}$. Consider the polynomial $q \Psi$,id of (3.4), with id the identity embedding of $F_{\mathfrak{p}}=\mathbb{Q}_{p^{d}}$ to $\overline{\mathbb{Q}}_{p}$. We have the factorization

$$
q_{\Psi, \text { id }}=c X^{2}+(d-a) X Y-b Y^{2}=A\left(X-\tau_{\Psi} Y\right)\left(X-\bar{\tau}_{\Psi} Y\right),
$$

where $A \in F_{\mathfrak{p}}^{\times}$, and $\tau_{\Psi}, \tau_{\Psi}^{\prime} \in \mathcal{H}\left(\mathbb{Q}_{p^{2 d}}\right)$ are the fixed points under the action of $\iota_{p}\left(\Psi\left(K_{\mathfrak{p}}^{\times}\right)\right)$on $\mathscr{H}\left(\mathbb{C}_{p}\right)$. We order $\tau_{\Psi}, \tau_{\Psi}^{\prime}$ in such a way that

$$
\iota_{p}(\Psi(\alpha))\left(\begin{array}{c}
\tau_{\Psi} \\
1
\end{array}\right)=\alpha\left(\begin{array}{c}
\tau_{\Psi} \\
1
\end{array}\right) \quad \text { for all } \alpha \in K_{\mathfrak{p}} .
$$

The element $Q_{\Psi}^{\frac{k-2}{2}} \in \mathscr{B}_{k}$ defines a function on $F_{\mathfrak{p}}^{2}$, where for $(x, y) \in F_{\mathfrak{p}}^{2}$, we have (in the notation of (3.6))

$$
Q_{\Psi}^{\frac{k-2}{2}}(x, y)=\left(\frac{\mathcal{N}_{F_{\mathfrak{p}} / \mathbb{Q}_{p} A}}{\sqrt{\left|\mathcal{N}_{F / \mathbb{Q}}\left(\lambda^{2}\right)\right|}}\right)^{\frac{k-2}{2}} \cdot \mathcal{N}_{\mathbb{Q}_{p^{2 d}} / \mathbb{Q}_{p}}(x-\tau \Psi y)^{\frac{k-2}{2}} .
$$

Definition 3.4. Given $\Xi \in S_{k}\left(\mathfrak{a}, \mathfrak{n}^{-}\right)$, and an optimal embedding $(\Psi, b)$, define the pairing

$$
\Xi[\Psi]=\left|\operatorname{Nrd}_{B / F}(b)\right|_{\hat{F}}^{\frac{k-2}{2}} \Xi(b)\left(Q_{\Psi}^{\frac{k-2}{2}} \mid b_{\mathfrak{p}}\right) .
$$

By equation (3.5), the value $\Xi[\Psi]$ depends only on the conjugacy class $[\Psi, b]$.

3.3. Special value formula of Rankin $\boldsymbol{L}$-functions. As in Section 3.1 , let $\psi_{K}$ be an anti-cyclotomic character over a CM extension $K$ of $F$. In this section, we state the formula of Xue [38], that generalizes the works of Gross [18], Hatcher [20], and Zhang [41]. We make some simplifying assumptions. Let $\Xi \in S_{k}\left(\mathfrak{a}, \mathfrak{n}^{-}\right)$be an eigenform, with $k \geq 2$ even, that corresponds, under the Jacquet-Langlands correspondence, to a Hilbert newform $\mathbf{g}$ of conductor $\mathfrak{n}=a \mathfrak{n}^{-}$. Assume that the conductor $\mathfrak{n}$ of $\mathbf{g}$ is relatively prime to $D_{K / F}$, the relative discriminant ideal of $K$ over $F$. Finally we assume that the character $\psi_{K}$ is unramified.

Definition 3.5. The algebraic part of the central $L$-value $L\left(k / 2, \mathbf{g} / K, \psi_{K}\right)$ is defined to be

$$
L^{\mathrm{alg}}\left(k / 2, \mathbf{g} / K, \psi_{K}\right):=u_{K}^{2} \frac{(k / 2-1) !^{2 d} \sqrt{\mathcal{N}_{F / \mathbb{Q}} D_{K / F}}}{(2 \pi)^{(k-2) d}\langle\mathbf{g}, \mathbf{g}\rangle} L\left(k / 2, \mathbf{g} / K, \psi_{K}\right) .
$$

Here $u_{K}=\left[\mathcal{O}_{K}^{\times}: \mathcal{O}_{F}^{\times}\right]$. The Peterson inner product $\langle\mathbf{g}, \mathbf{g}\rangle$ is normalized as follows:

$$
\langle\mathbf{g}, \mathbf{g}\rangle=\left(8 \pi^{2}\right)^{d} \int_{\mathbf{A}_{F}^{\times} \mathrm{GL}_{2}(F) \backslash \mathrm{GL}_{2}\left(\mathbf{A}_{F}\right)}|\mathbf{g}|^{2} d h
$$


with $d h$ the measure on $\mathrm{GL}_{2}\left(\mathbf{A}_{F}\right)$ which induces the usual hyperbolic measure on products of the Poincaré upper half plane, and normalized by the condition

$$
\text { volume of } K_{\infty} I_{\mathbf{A}_{F}}(\mathfrak{n})=1,
$$

where $K_{\infty}=\prod_{\nu \mid \infty} \mathrm{SO}_{2}(\mathbb{R})$, and $I_{\mathbf{A}_{F}}(\mathfrak{n})=\prod_{\mathfrak{r}} I_{\Upsilon^{\text {val }} \mathfrak{r} \mathfrak{r}}$.

The Petersson inner product on the space of quaternionic forms $S_{k}\left(\mathfrak{a}, \mathfrak{n}^{-}\right)$is defined as in Section 2.2 of [4]; see also Section 18 of [19]. Let $U \subset B$ be the $F$-subspace of dimension 3 consisting of elements of reduced trace zero, with right action of $B^{\times}$on $U$ given by

$$
u \cdot b=b^{-1} u b .
$$

Define the non-degenerate $B^{\times}$-invariant symmetric bilinear pairing

$$
\begin{gathered}
{[\cdot, \cdot]: U \times U \rightarrow F,} \\
{[u, v]=\frac{1}{2} \operatorname{Trd}_{B / F}(u \bar{v}),}
\end{gathered}
$$

where $\operatorname{Trd}_{B / F}$ denotes the reduced trace of $B$, and $\bar{v}$ denotes the image of $v$ under the canonical involution of $B$. For $\sigma: F_{\mathfrak{p}} \hookrightarrow \overline{\mathbb{Q}}_{p}$, there is an isomorphism

$$
U \otimes_{F, \sigma} \mathbb{C}_{p} \stackrel{\sim}{\longrightarrow} \operatorname{Sym}_{\sigma}^{2}
$$

sending $\left(\begin{array}{ll}a & b \\ c & d\end{array}\right) \in U \otimes_{F, \sigma} \mathbb{C}_{p}$ to the element $c^{\sigma}\left(X^{\sigma}\right)^{2}+\left(d^{\sigma}-a^{\sigma}\right) X^{\sigma} Y^{\sigma}-b^{\sigma}\left(Y^{\sigma}\right)^{2}$ (cf. equation (3.4)); this isomorphism is $B_{1}^{\times}$-equivariant, where $B_{1}^{\times}$is the subgroup of $B^{\times}$of reduced norm one, acting on $\operatorname{Sym}_{\sigma}^{2}$ via its image in $B_{\mathfrak{p}} \stackrel{\iota_{\mathfrak{p}}}{=} \mathrm{GL}_{2}\left(F_{\mathfrak{p}}\right)$ (there would be a twist by the inverse of the determinant on the right hand side, if one considers the full action of $B^{\times}$). For $k \geq 2$, even, we have a natural surjection

$$
\mathrm{Sym}^{k / 2-1}\left(\mathrm{Sym}_{\sigma}^{2}\right) \rightarrow \mathrm{Sym}_{\sigma}^{k-2},
$$

hence we have a surjection

$$
\otimes_{\sigma: F_{\mathfrak{p}} \hookrightarrow \overline{\mathbb{Q}}_{p}}\left(\operatorname{Sym}^{k / 2-1}\left(U \otimes_{F, \sigma} \mathbb{C}_{p}\right)\right) \rightarrow \otimes_{\sigma: F_{\mathfrak{p}} \hookrightarrow \overline{\mathbb{Q}}_{p}} \operatorname{Sym}_{\sigma}^{k-2}=\mathscr{B}_{k} .
$$

Upon dualizing, this induces the injection

$$
V_{k} \hookrightarrow \otimes_{\sigma: F_{\mathfrak{p}} \hookrightarrow \overline{\mathbb{Q}}_{p}}\left(\operatorname{Sym}^{k / 2-1}\left(U \otimes_{F, \sigma} \mathbb{C}_{p}\right)\right)^{*}
$$

(where $\left(\operatorname{Sym}^{k / 2-1}\left(U \otimes_{F, \sigma} \mathbb{C}_{p}\right)\right)^{*}$ denotes the $\mathbb{C}_{p}$-dual of Sym ${ }^{k / 2-1}\left(U \otimes_{F, \sigma} \mathbb{C}_{p}\right)$.

Now the symmetric $F$-bilinear pairing $[\cdot, \cdot]$ on $U$ induces the symmetric $\mathbb{C}_{p^{-}}$ bilinear pairing $[\cdot, \cdot]_{\sigma}$ on $U \otimes_{F, \sigma} \mathbb{C}_{p}$, which can be extended to $\operatorname{Sym}^{k / 2-1}\left(U \otimes_{F, \sigma} \mathbb{C}_{p}\right)$ 
by

$$
\begin{aligned}
& {\left[\left(u_{1}, \ldots, u_{k / 2-1}\right),\left(v_{1}, \ldots, v_{k / 2-1}\right)\right]_{k, \sigma}} \\
& \quad=\sum_{\eta \in S_{k / 2-1}}\left[u_{1}, v_{\eta(1)}\right]_{\sigma} \ldots\left[u_{k / 2-1}, v_{\eta(k / 2-1)}\right]_{\sigma}
\end{aligned}
$$

with $S_{k / 2-1}$ the symmetric group on $k / 2-1$ letters. The non-degenerate pairings $[\cdot, \cdot]_{k, \sigma}$ allow us to identify $\operatorname{Sym}^{k / 2-1}\left(U \otimes_{F, \sigma} \mathbb{C}_{p}\right)$ with its dual. We still denote by $[\cdot, \cdot]_{k, \sigma}$ the inner product induced on $\left(\operatorname{Sym}^{k / 2-1}\left(U \otimes_{F, \sigma} \mathbb{C}_{p}\right)\right)^{*}$, and by $[\cdot, \cdot]_{k}=$ $\otimes_{\sigma}[\cdot, \cdot]_{k, \sigma}$ the tensor inner product induced on $\otimes_{\sigma: F_{\mathfrak{p}} \hookrightarrow \overline{\mathbb{Q}}_{p}}\left(\operatorname{Sym}^{k / 2-1} U \otimes_{F, \sigma} \mathbb{C}_{p}\right)^{*}$. Its restriction to $V_{k}$ is still noted as $[\cdot, \cdot]_{\underline{k}}$. By construction, it is $B_{1}^{\times}$-invariant.

Now given $\Xi_{1}, \Xi_{2} \in S_{k}\left(a, \mathfrak{n}^{-}\right)$, define

$$
\left\langle\Xi_{1}, \Xi_{2}\right\rangle=\sum_{b \in B^{\times} \backslash \widehat{B}^{\times} / \Sigma\left(\mathfrak{a}, \mathfrak{n}^{-}\right)} \frac{1}{e_{b}}\left|\operatorname{Nrd}_{B / F}(b)\right|_{\hat{F}}^{k-2}\left[b_{\mathfrak{p}} \cdot \Xi_{1}(b), b_{\mathfrak{p}} \cdot \Xi_{2}(b)\right]_{\underline{k}},
$$

where for $b \in \widehat{B}^{\times}$, we denote by $e_{b}$ the order of the group $R_{b}^{\times} / \mathcal{O}_{F}^{\times}$, with $R_{b}=$ $B^{\times} \cap b \Sigma\left(a, \mathfrak{n}^{-}\right) b^{-1}$ the Eichler order of level a determined by $g$ (each summand depends only on the image of $b$ in the double coset).

We now state the main formula of [38].

Theorem 3.6 ([38], Theorem 1.2). Under the assumptions made in the beginning of this section,

$$
L^{\mathrm{alg}}\left(k / 2, \mathbf{g} / K, \psi_{K}\right)=\frac{1}{\langle\Xi, \Xi\rangle}\left|\sum_{\rho \in \operatorname{Pic}\left(\mathcal{O}_{K}\right)} \psi_{K}(\rho) \Xi\left[\Psi^{\rho}\right]\right|^{2} .
$$

Here the absolute value in (3.10) is interpreted as

$$
\left|\sum_{\rho \in \operatorname{Pic}\left(\mathcal{O}_{K}\right)} \psi_{K}(\rho) \Xi\left[\Psi^{\rho}\right]\right|^{2}=\left(\sum_{\rho \in \operatorname{Pic}\left(\mathcal{O}_{K}\right)} \psi_{K}(\rho) \Xi\left[\Psi^{\rho}\right]\right) \cdot\left(\sum_{\rho \in \operatorname{Pic}\left(\mathcal{O}_{K}\right)} \psi_{K}^{-1}(\rho) \Xi\left[\Psi^{\rho}\right]\right) .
$$

We remark that in [38], the unitary normalization of automorphic $L$-function is used, so that in loc. cit. the central $L$-value occurs when $s=1 / 2$, and the Rankin $L$-function that occurs there is the completed one:

$$
(2 \pi)^{-2 d\left(s+\frac{k-2}{2}\right)} \Gamma\left(s+\frac{k-2}{2}\right)^{2 d} L\left(s+\frac{k-2}{2}, \mathbf{g} / K, \psi_{K}\right) .
$$

We also note that the normalization of the Petersson inner product of Hilbert modular forms in Definition 3.5 above is different from [38], namely that the factor $\left(8 \pi^{2}\right)^{d}$ does not occur in loc. cit.

One corollary of Xue's formula is the following algebraicity result: 
Corollary 3.7 ([38], Proposition 3.18). The value $L^{\mathrm{alg}}\left(k / 2, \mathbf{g} / K, \psi_{K}\right)$ lies in $\mathbb{Q}\left(\mathbf{g}, \psi_{K}\right)$, where $\mathbb{Q}\left(\mathbf{g}, \psi_{K}\right)$ is the number field generated by the normalized Fourier coefficients of $\mathbf{g}$ and the values of $\psi_{K}$. More precisely, for any $h \in \operatorname{Aut}(\overline{\mathbb{Q}})$, we have

$$
L^{\operatorname{alg}}\left(k / 2, \mathbf{g} / K, \psi_{K}\right)^{h}=L^{\operatorname{alg}}\left(k / 2, \mathbf{g}^{h} / K, \psi_{K}^{h}\right) .
$$

Here $\mathbf{g}^{h}$ is the cuspidal Hilbert eigenform that satisfies $C\left(\mathfrak{q}, \mathbf{g}^{h}\right)=C(\mathfrak{q}, \mathbf{g})^{h}$, and $\psi_{K}^{h}=h \circ \psi_{K}[31]$.

Remark 3.8. A more general special value formula, valid for more general ring class characters $\psi_{K}$ of $K$, was proved in the thesis of H. P. Yuan [39] (see also the recent work of B. Howard [23]).

In the rest of the paper, we only consider the case where $\psi_{K}$ is a genus character.

3.4. $p$-adic interpolation. We now interpolate the algebraic part of the special values of the Rankin $L$-functions, using (3.10). In the rest of the section, the level a will be taken to be $\mathfrak{n}^{+}$, so $\Sigma=\Sigma\left(\mathfrak{n}^{+}, \mathfrak{n}^{-}\right)$as in Section 2.4.

We first give another description of optimal embeddings. Recall the statement of strong approximation (2.14): for the fixed choice of elements $\left\{x_{i}\right\}_{i=1}^{h} \subset \widehat{B}^{\times}$, with $\left(x_{i}\right)_{\mathfrak{p}}=1$, we have a decomposition

$$
\bigsqcup_{i=1}^{h} \widetilde{\Gamma}_{i} \backslash B_{\mathfrak{p}}^{\times} / R_{\mathfrak{p}}^{\times} \stackrel{\sim}{\longrightarrow} B^{\times} \backslash \widehat{B}^{\times} / \widehat{R}^{\times}
$$

sending the class of an element $g \in B_{\mathfrak{p}}^{\times}$of the $i$-th component on the left to the double coset on the right defined by $x_{i} g$.

Hence we can identify $\operatorname{Emb}_{F}\left(K, B, \mathfrak{n}^{+}\right)$, the set of conjugacy classes of optimal embeddings of level $\mathfrak{n}^{+}$, as a subset of elements of

$$
\begin{aligned}
\bigsqcup_{i=1}^{h} & \widetilde{\Gamma}_{i} \backslash\left(\operatorname{Hom}_{\mathcal{O}_{F}}\left(\mathcal{O}_{K}\left[\frac{1}{p}\right], R_{i}\left[\frac{1}{p}\right]\right) \times B_{\mathfrak{p}}^{\times} /\left(R_{i}\right)_{\mathfrak{p}}^{\times}\right) \\
& \stackrel{\iota_{\mathfrak{p}}}{\cong} \bigsqcup_{i=1}^{h} \widetilde{\Gamma}_{i} \backslash\left(\operatorname{Hom}_{\mathcal{O}_{F}}\left(\mathcal{O}_{K}\left[\frac{1}{p}\right], R_{i}\left[\frac{1}{p}\right]\right) \times \operatorname{GL}_{2}\left(F_{\mathfrak{p}}\right) / \mathrm{GL}_{2}\left(\mathcal{O}_{F_{\mathfrak{p}}}\right)\right),
\end{aligned}
$$

where $R_{i}=B \cap x_{i} \widehat{R} x_{i}^{-1}$.

Write $[\Psi, g]_{i}$ for a conjugacy class of optimal embedding, identified as an element that belongs to the $i$-th component of (3.11). Pick a representative $(\Psi, g)_{i}$ for the class $[\Psi, g]_{i}$. Define $L_{\Psi}:=g\left(\mathcal{O}_{F_{\mathfrak{p}}} \oplus \mathcal{O}_{F_{\mathfrak{p}}}\right)$. By the optimality condition, and that $\left(x_{i}\right)_{\mathfrak{p}}=1$, one sees that $L_{\Psi}$ is stable under the action of $\iota_{p}\left(\Psi\left(\mathcal{O}_{K} \otimes \mathbb{Z}_{p}\right)\right)$. Define $\left|L_{\Psi}\right|$, the generalized index of $L_{\Psi}$, as $|\operatorname{det} g|_{\widehat{F}}^{-1}=q^{\operatorname{ord}_{p} \operatorname{det} g}$. 
In this notation, if $[\Psi]=[\Psi, g]_{i}$, and $\Xi$ corresponds to the $h$-tuple $\left(\xi^{1}, \ldots, \xi^{h}\right)$, then

$$
\Xi[\Psi]=\left|\operatorname{Nrd}_{B / F} x_{i}\right|_{\widehat{F}}^{\frac{k-2}{2}}\left|L_{\Psi}\right|^{\frac{2-k}{2}} c_{\xi^{i}}\left(L_{\Psi}\right)\left(Q_{\Psi}^{\frac{k-2}{2}}\right) .
$$

In particular, the expression on the right hand side of (3.12) depends only on the class $[\Psi, g]_{i}$.

Remark 3.9. If $\mathfrak{p}$ is inert in $K$, then $L_{\Psi}$ and $L_{\tau_{\Psi}}$ are homothetic. Indeed, their homothety classes define the unique fixed point of the action of $\iota_{p}\left(\Psi\left(K_{\mathfrak{p}}^{\times}\right)\right)$on the Bruhat-Tits tree. Also notice that the image of $\tau_{\Psi}$ in $\widetilde{\Gamma}_{i} \backslash \mathscr{H}\left(\mathbb{Q}_{p^{2 d}}\right)=\Gamma_{i} \backslash \mathscr{H}^{\prime}\left(\mathbb{Q}_{p^{2 d}}\right)$ depends only on the class $[\Psi, g]_{i}$.

Definition 3.10. Define the region $L_{\Psi}^{\prime \prime} \subset L_{\Psi}$ as follows. If $\mathfrak{p}$ is inert in $K$, then $L_{\Psi}^{\prime \prime}:=L_{\Psi}^{\prime}$. While if $\mathfrak{p}$ splits in $K$, then $L_{\Psi}$ admits a basis $\left(v_{1}, v_{2}\right)$ consisting of eigenvectors under the action of $\iota_{p}\left(\Psi\left(\mathcal{O}_{K} \otimes \mathbb{Z}_{p}\right)\right)$. We define $L_{\Psi}^{\prime \prime}:=\mathcal{O}_{F_{\mathfrak{p}}}^{\times} v_{1} \times \mathcal{O}_{F_{\mathfrak{p}}}^{\times} v_{2}$.

\section{Lemma 3.11.}

$$
\operatorname{ord}_{p}\left(Q_{\Psi}(x, y)\right)=\operatorname{ord}_{p}\left|L_{\Psi}\right| \quad \text { for }(x, y) \in L_{\Psi}^{\prime \prime} .
$$

Proof. Since $\mathfrak{p}=p \mathcal{O}_{F}$ is unramified in $K$, we can write $K=F(\lambda)$, with $\lambda^{2} \in$ $F$ totally negative, and $\operatorname{ord}_{p} \lambda^{2}=0$. As above $L_{\Psi}=g\left(\mathcal{O}_{F_{\mathfrak{p}}}^{2}\right)$. Then as in [4], Lemma 3.7, one can show, for any $\sigma \in \operatorname{Hom}\left(F_{\mathfrak{p}}, \overline{\mathbb{Q}}_{p}\right)$, that

$$
\operatorname{ord}_{p} q \Psi, \sigma\left(x^{\sigma}, y^{\sigma}\right)=\operatorname{ord}_{p} \operatorname{det} g \text { on } L_{\Psi}^{\prime \prime} \text {. }
$$

Hence on $L_{\Psi}^{\prime \prime}$ one has

$$
\operatorname{ord}_{p} Q_{\Psi}(x, y)=\sum_{\sigma: F_{\mathfrak{p}} \hookrightarrow \overline{\mathbb{Q}}_{p}} \operatorname{ord}_{p} q_{\Psi, \sigma}\left(x^{\sigma}, y^{\sigma}\right)=\sum_{\sigma: F_{\mathfrak{p}} \hookrightarrow \overline{\mathbb{Q}}_{p}} \operatorname{ord}_{p} \operatorname{det} g=\operatorname{ord}_{p}\left|L_{\Psi}\right| .
$$

Definition 3.12. We refer to the notation of Sections 2.5 and 2.7. Associated to the Hida family $\mathbf{f}_{\infty}$, and optimal embedding $[\Psi]=[\Psi, g]_{i}$ of level $\mathfrak{n}^{+}$, define the $p$-adic analytic function $\mathscr{L}_{p}\left(\mathbf{f}_{\infty} / K, \Psi, k\right)$ of the variable $k \in U$ :

$$
\mathscr{L}_{p}\left(\mathbf{f}_{\infty} / K, \Psi, k\right):=\left\langle\left|\operatorname{Nrd}_{B / F} x_{i}\right|_{\widehat{F}}\right\rangle^{\frac{k-2}{2}} \int_{\mathfrak{c} \backslash L_{\Psi}^{\prime \prime}}\left\langle Q_{\Psi}^{\frac{k-2}{2}}\right\rangle d \mu_{i, L_{\Psi}} .
$$

The function $\mathscr{L}_{p}\left(\mathbf{f}_{\infty} / K, \Psi, k\right)$ depends only on the class [ $\left.\Psi, g\right]_{i}$ by equation (3.5).

Given a genus character $\psi_{K}$ of $K$, define

$$
\mathscr{L}_{p}\left(\mathbf{f}_{\infty} / K, \psi_{K}, k\right):=\sum_{\rho \in \operatorname{Pic}\left(\mathcal{O}_{K}\right)} \psi_{K}(\rho) \mathscr{L}_{p}\left(\mathbf{f}_{\infty} / K, \Psi^{\rho}, k\right) .
$$


We have the interpolation formula:

Theorem 3.13. Suppose that $\mathfrak{p}$ is inert in $K$. Then for $k \in U \cap \mathbb{Z}^{>2}, k \equiv 2$ $\bmod 2(p-1)$,

$$
\mathscr{L}_{p}\left(\mathbf{f}_{\infty} / K, \psi_{K}, k\right)^{2}=\left\langle\Phi_{k}^{\#}, \Phi_{k}^{\#}\right\rangle \alpha(\mathfrak{p}, k)^{2}\left(1-\frac{q^{k-2}}{\alpha(\mathfrak{p}, k)^{2}}\right)^{2} L^{\mathrm{alg}}\left(k / 2, \mathbf{f}_{k}^{\#} / K, \psi_{K}\right)
$$

For $k=2$ we have $\mathscr{L}_{p}\left(\mathbf{f}_{\infty} / K, \psi_{K}, 2\right)=0$.

Proof. When $k>2$ this follows by combining Proposition 2.15, Theorem 3.6, Lemma 3.11 and equation (3.12). That $\mathscr{L}_{p}\left(\mathbf{f}_{\infty} / K, \psi_{K}, 2\right)=0$ follows from equation (2.19).

Note: The function $\mathscr{L}_{p}\left(\mathbf{f}_{\infty} / K, \psi_{K}, k\right)$ essentially interpolates the square root of the algebraic part of the central $L$-values $L^{\mathrm{alg}}\left(k / 2, \mathbf{f}_{k}^{\#}, \psi_{K}\right)$ along the Hida family, hence the name square root $p$-adic L-function in [4].

We need a corresponding result, when $\mathfrak{p}$ splits in $K$ :

Theorem 3.14. Suppose that $\mathfrak{p}$ splits in $K$. Then if $\psi_{K}$ corresponds to a pair $\left(\psi_{1}, \psi_{2}\right)$ of Hecke characters of $F$, we have

$$
\mathscr{L}_{p}\left(\mathbf{f}_{\infty} / K, \psi_{K}, 2\right)^{2}=\langle\Phi, \Phi\rangle\left(1-\frac{\psi_{1}(\mathfrak{p})}{\alpha\left(\mathfrak{p}, \mathbf{f}_{2}\right)}\right)^{2} L^{\mathrm{alg}}\left(1, \mathbf{f}_{2} / K, \psi_{K}\right)
$$

and for $k \in U \cap \mathbb{Z}^{>2}, k \equiv 2 \bmod 2(p-1)$,

$$
\begin{aligned}
& \mathscr{L}_{p}\left(\mathbf{f}_{\infty} / K, \psi_{K}, k\right)^{2} \\
& \quad=\left\langle\Phi_{k}^{\#}, \Phi_{k}^{\#}\right\rangle \alpha(\mathfrak{p}, k)^{2}\left(1-\frac{\psi_{1}(\mathfrak{p}) q^{\frac{k-2}{2}}}{\alpha(\mathfrak{p}, k)}\right)^{4} L^{\operatorname{alg}}\left(k / 2, \mathbf{f}_{k}^{\#} / K, \psi_{K}\right) .
\end{aligned}
$$

Proof. The proof is the same as in [4], Theorem 3.12, again using Proposition 2.15, Theorem 3.6, and Lemma 3.11.

Remark 3.15. Using the more general formula of [39] for the special values of Rankin $L$-functions, it should be possible to construct the two-variable anti-cyclotomic $p$ adic $L$-function, attached to the Hida family $\mathbf{f}_{\infty}$ and ring class characters of the CM extension $K / F$. Then the function $\mathscr{L}_{p}\left(\mathbf{f}_{\infty} / K, \psi_{K}, k\right)$ would just be the special value of the two-variable $p$-adic $L$-function, evaluated at central critical points and at the unramified character $\psi_{K}$. 
Suppose now that $\mathfrak{p}$ is inert in $K$. Corresponding to $[\Psi]$ and the genus character $\psi_{K}$, we form the divisor

$$
P_{\psi_{K}}=\sum_{\rho \in \operatorname{Pic}\left(\mathcal{O}_{K}\right)} \psi_{K}(\rho) \tau_{\Psi^{\rho}} \in \bigoplus_{i=1}^{h} \operatorname{Div}\left(\Gamma_{i} \backslash \mathcal{H}^{\prime}\left(\mathbb{Q}_{p^{2 d}}\right)\right)
$$

Here the divisor of $\tau_{\Psi^{\rho}}$ lies in the $i$-th component, if $i$ is the index of the component of (3.11) to which $\left[\Psi^{\rho}\right]$ belongs.

Proposition 3.16. In the above notations, we have

$$
\left.\frac{d}{d k} \mathscr{L}_{p}\left(\mathbf{f}_{\infty} / K, \psi_{K}, k\right)\right|_{k=2}=\frac{1}{2} I_{\Phi}\left(P_{\psi_{K}}\right)
$$

Proof. Suppose that $i_{\Psi}$ is the index of the component of ((3.11) to which $[\Psi]$ belongs. From (3.13) and (3.8), we see, by comparing with (2.23), that

$$
\begin{aligned}
\left.\frac{d}{d k} \mathscr{L}_{p}\left(\mathbf{f}_{\infty} / K, \Psi, k\right)\right|_{k=2} & =\frac{1}{2} \int_{\mathfrak{c} \backslash L_{\tau_{\Psi}}^{\prime}} \log _{p} F_{\tau_{\Psi}}(x, y) d \mu_{i_{\Psi}, L_{\tau} \Psi}(x, y) \\
& =\frac{1}{2} I_{\phi^{i} \Psi}\left(\tau_{\Psi}\right) .
\end{aligned}
$$

Similar equations hold with $\Psi$ replaced with $\Psi^{\rho}$. The proposition thus follows by linearity.

\section{Heegner points on Shimura curves}

4.1. Shimura curves. In this section, we define the Shimura curves that will be used in the sequel. For more details, see [10], [40].

Fix an archimedean place $v_{0}$ of $F$. Denote by $\mathcal{B} / F$ the quaternion algebra over $F$, obtained from $B / F$ by switching the invariants at $v_{0}$ and $\mathfrak{p}$. Thus the invariants of $\mathcal{B}$ and that of $B$ are related as follows:

$$
\begin{aligned}
\operatorname{inv}_{v_{0}} \mathcal{B} & =\operatorname{inv}_{\mathfrak{p}} B=0 \\
\operatorname{inv}_{\mathfrak{p}} \mathcal{B} & =\operatorname{inv}_{v_{0}} B=1 / 2, \\
\operatorname{inv}_{\boldsymbol{v}} \mathcal{B} & =\operatorname{inv}_{\boldsymbol{v}} B \quad \text { for } v \neq v_{0}, \mathfrak{p} .
\end{aligned}
$$

Fix an isomorphism $\mathscr{B} \otimes_{v_{0}} \mathbb{R} \cong M_{2}(\mathbb{R})$, so that $\left(\mathcal{B} \otimes_{v_{0}} \mathbb{R}\right)^{\times}$can be identified as $\mathrm{GL}_{2}(\mathbb{R})$.

Let $\mathcal{O}_{\mathscr{B}}$ be a maximal order of $\mathcal{B}$, and let $\mathcal{R} \subset \mathcal{O}_{\mathscr{B}}$ be an Eichler order of level $\mathfrak{n}^{+}$contained in $\mathcal{O}_{\mathscr{B}}$. By Shimura's theory, associated to $\mathscr{B}$ and the level $\mathfrak{n}^{+}$is 
the Shimura curve $X_{\mathcal{B}}\left(\mathfrak{n}^{+}\right)$, which is a proper smooth curve defined over $F$ (not geometrically connected in general), whose $\mathbb{C}$-points (with respect to $\nu_{0}: F \hookrightarrow \mathbb{C}$ ) are given by the double coset

$$
X_{\mathcal{B}}\left(\mathfrak{n}^{+}\right)(\mathbb{C})=\mathscr{B}^{\times} \backslash(\mathbb{C}-\mathbb{R}) \times \widehat{\mathcal{B}}^{\times} / \widehat{\mathcal{R}}^{\times}
$$

(here $\mathscr{B}^{\times}$acts on $\mathbb{C}-\mathbb{R}$ via $\mathscr{B}^{\times} \rightarrow\left(\mathscr{B} \otimes_{\mathcal{V}_{0}} \mathbb{R}\right)^{\times} \cong \mathrm{GL}_{2}(\mathbb{R})$, with $\mathrm{GL}_{2}(\mathbb{R})$ acting on $\mathbb{C}-\mathbb{R}$ by Möbius transformation). The geometric components of $X_{\mathcal{B}}\left(\mathfrak{n}^{+}\right)$are defined over the strict Hilbert class field of $F$, i.e., the abelian extension of $F$ corresponding to the strict ideal class group $\mathrm{Cl}_{F}$ of $\mathcal{O}_{F}$. As in (2.12), let $h$ be the order of the $\mathrm{Cl}_{F}$. Then the geometric components are indexed by $\mathrm{Cl}_{F}$, and the number of such components is $h$.

The (disconnected) Riemann surface (4.1) admits a more classical description as follows. First, denote by $\mathscr{B}_{+}$the set of elements of $\mathscr{B}$ whose reduced norm to $F$ is totally positive (this is equivalent to saying that the reduced norm is positive under the embedding $v_{0}$, as $\mathscr{B}$ is ramified at other archimedean places). Let $y_{1}, \ldots, y_{h} \in \widehat{\mathscr{B}}^{\times}$ be a set of representatives of

$$
\mathcal{B}_{+}^{\times} \backslash \widehat{\mathcal{B}}^{\times} / \hat{\mathcal{R}}^{\times}
$$

Define

$$
\Delta_{i}=\mathscr{B}_{+}^{\times} \cap y_{i} \widehat{\mathcal{R}}^{\times} y_{i}^{-1} .
$$

Then if $\mathfrak{h}$ is denotes the Poincaré upper half plane, we have

$$
X_{\mathcal{B}}\left(\mathfrak{n}^{+}\right)(\mathbb{C})=\bigsqcup_{i=1}^{h} \Delta_{i} \backslash \mathfrak{h} .
$$

When $F \neq \mathbb{Q}$, the Shimura curve $X_{\mathcal{B}}\left(\mathfrak{n}^{+}\right)$is not of PEL type. To describe a PEL Shimura curve associated to $X_{\mathscr{B}}\left(\mathfrak{n}^{+}\right)$, we need to consider base change to an auxiliary CM extension of $F$. We recall the constructions [10], [40].

Fix a auxiliary CM extension $M / F$ of the form $M=F(\sqrt{r})$, where $r$ is a negative integer. Assume that all the primes dividing $\mathfrak{n}^{+}$splits in $M$. Extend the embedding $v_{0}: F \hookrightarrow \mathbb{R}$ to an embedding of $M$ to $\mathbb{C}$ by

$$
v_{0}(x+y \sqrt{r})=v_{0}(x)+\sqrt{r} v_{0}(y) \text { for } x, y \in F .
$$

Let $\mathfrak{D}=\mathscr{B} \otimes_{F} M$, and denote by $V$ be the underlying $\mathbb{Q}$-vector space $\mathfrak{D}$, with the left $\mathfrak{D}$-action. Denote $V_{\mathbb{R}}:=V \otimes_{\mathbb{Q}} \mathbb{R}$. So we can identify:

$$
V_{\mathbb{R}}=\left(\mathscr{B} \otimes_{F} M\right) \otimes_{\mathbb{Q}} \mathbb{R}=M_{2}(\mathbb{C}) \oplus\left(\mathbb{H} \otimes_{\mathbb{R}} \mathbb{C}\right)^{d-1},
$$


where $\mathbb{H}$ is the set of Hamilton's quaternions, and the projection to the first factor corresponds to the isomorphism $B \otimes_{F, \nu_{0}} \mathbb{C} \cong M_{2}(\mathbb{C})$. $V_{\mathbb{R}}$ has a complex structure, with multiplication by $\sqrt{-1}$ given by right multiplication on $V_{\mathbb{R}}$ by the element

$$
j=\left(\left(\begin{array}{cc}
0 & 1 \\
-1 & 0
\end{array}\right), 1 \otimes \sqrt{-1}, \ldots, 1 \otimes \sqrt{-1}\right) .
$$

It follows that the action of $\mathfrak{D}$ on $V_{\mathbb{R}}$ is $\mathbb{C}$-linear. It can be shown that, with respect to this complex structure, $\operatorname{tr}\left(l, V_{\mathbb{R}} / \mathbb{C}\right) \in M$ for all $l \in \mathfrak{D}$.

Let $l \rightarrow \bar{l}$ be the involution of $\mathfrak{D}$ obtained by tensoring the involution of $\mathscr{B}$ and the conjugation of $M$ over $F$. Let $G$ be the algebraic group over $F$ defined by the condition that for any $F$-algebra $T$,

$$
G(T)=\left\{l \in\left(\mathfrak{D} \otimes_{F} T\right)^{\times} \text {such that } l \bar{l} \in T^{\times}\right\} .
$$

Define the level subgroups $\Sigma_{M} \subset G(\widehat{F})$ by

$$
\Sigma_{M}=\widehat{\mathcal{R}}^{\times} \cdot \widehat{\mathcal{O}}_{M}^{\times} .
$$

Then by Shimura's theory, there is a proper smooth curve $X_{\mathfrak{D}}\left(\mathfrak{n}^{+}\right)$defined over $M$, whose set of $\mathbb{C}$-points is given by the double coset

$$
X_{\mathfrak{D}}\left(\mathfrak{n}^{+}\right)(\mathbb{C})=G(F) \backslash(\mathbb{C}-\mathbb{R}) \times G(\widehat{F}) / \Sigma_{M} .
$$

The geometric components of $X_{\mathfrak{D}}\left(\mathfrak{n}^{+}\right)$are defined over the Hilbert class field of $M$. There is a map $X_{\mathscr{B}}\left(\mathfrak{n}^{+}\right) \hookrightarrow X_{\mathfrak{D}}\left(\mathfrak{n}^{+}\right)$, which is both open and closed immersion, and defined over the Hilbert class field of $M$, which at the level of $\mathbb{C}$-points is the natural map from the double coset of (4.1) to that of (4.3).

One can also describe $G$ as the group of symplectic similitudes of an alternating form as follows.

For any invertible element $\delta \in \mathfrak{D}$, which is symmetric, i.e., $\bar{\delta}=\delta$, we can define another involution on $\mathfrak{D}$, noted as $l \rightarrow l^{*}$, by

$$
l^{*}=\delta^{-1} \bar{l} \delta .
$$

For $v, w \in V$ define

$$
p_{F}(v, w):=\operatorname{Tr}_{M / F}\left(\sqrt{r} \operatorname{Trd}_{\mathfrak{D} / M}\left(v \delta w^{*}\right)\right) .
$$

Then $p_{F}$ is an $F$-valued non-degenerate alternating form, satisfying

$$
p_{F}(l v, w)=p_{F}\left(v, l^{*} w\right) .
$$

Choose a $\delta$, so that the form $p_{F}(\cdot, \cdot j)$ is positive definite on $V_{\mathbb{R}}$, in which case the involution $l \rightarrow l^{*}$ is a positive involution. One can show that the algebraic group $G$ 
defined above is isomorphic to the group of $\mathfrak{D}$-linear symplectic similitudes of the form $p_{F}$.

The curve $X_{\mathfrak{D}}\left(\mathfrak{n}^{+}\right)$is a coarse moduli space for abelian varieties whose endomorphism algebras contain $\mathfrak{D}$. More precisely, let $\mathcal{O}_{\mathfrak{D}}:=\mathcal{O}_{\mathfrak{B}} \otimes \mathcal{O}_{M}$, which is a maximal order of $\mathfrak{D}$, and let $V_{\mathbb{Z}}:=\mathcal{O}_{\mathfrak{D}}$, regarded as a lattice of $V$. Then $X_{\mathfrak{D}}$ coarsely represents the functor $\mathcal{F}$, which associates to a scheme $S$ over $M$, the isomorphism classes of objects $[A, \iota, \bar{\theta}, \bar{\kappa}, C]$, where

- $A$ is an abelian scheme over $S$ of relative dimension $4[F: \mathbb{Q}]$, with an action $\iota: \mathcal{O}_{\mathfrak{D}} \rightarrow \operatorname{End}_{S}(A)$ such that for any $l \in \mathcal{O}_{\mathfrak{D}}$,

$$
\operatorname{tr}(\iota(l): \text { Lie } A)=\operatorname{tr}\left(l, V_{\mathbb{R}} / \mathbb{C}\right) ;
$$

- $\bar{\theta}$ is an $\mathcal{O}_{F}^{\times}$-class of polarizations $\theta: A \rightarrow \check{A}$ from $A$ to the dual abelian scheme $\check{A}$, such that for any $l \in \mathcal{O}_{\mathfrak{D}}$, the associated Rosati involution takes $\iota(l)$ to $\iota\left(l^{*}\right)$ (recall that if $\phi \in \operatorname{End}(A)$, then the Rosati involution defined by a polarization $\theta$ is given by $\phi^{*}:=\theta^{-1} \check{\phi} \theta$, where $\check{\phi} \in \operatorname{End}(\check{A})$ is the dual of $\phi$ );

- $\bar{\kappa}$ is a $\Sigma_{M}$-class of $\mathcal{O}_{\mathfrak{D}}$-linear isomorphisms $\kappa: \widehat{V}_{\mathbb{Z}} \rightarrow \widehat{T}(A)$ (where $\widehat{T}(A)$ is the adelic Tate module of $A$ ), which is symplectic with respect to the form $p_{u}:=\operatorname{tr}_{\widehat{F} / \widehat{\mathbb{Q}}}\left(u \cdot p_{F}\right)$ on $\widehat{V}_{\mathbb{Z}}$ for some $u \in \widehat{F}^{\times}$, and the Riemann form $p_{A}$ on $\widehat{T}(A)$ induced by a polarization $\theta \in \bar{\theta}$ :

- $C$ is a subgroup scheme of $A$, locally isomorphic to $\mathcal{O}_{F} / \mathfrak{n}^{+}$, and which is stable and locally cyclic under the action of $\mathcal{R}$ induced by $\iota$.

In the following section we will make the abbreviation by referring to such an object as $[A, C]$.

4.2. CM points. In this section we recall the basic definitions about $\mathrm{CM}$ points. For details, see [40].

As in Section 3, let $K / F$ be a CM extension. For the rest of Section 4, assume that the primes dividing $\mathfrak{p} \mathfrak{n}^{-}$are inert in $K$, and the primes dividing $\mathfrak{n}^{+}$split in $K$.

Similar to Definition 3.1, an optimal embedding of $K$ into $\mathscr{B}$ of level $\mathfrak{n}^{+}$is a pair $(\widetilde{\Psi}, c) \in \operatorname{Hom}_{F}(K, \mathscr{B}) \times \widehat{\mathcal{B}}^{\times} / \widehat{\mathcal{R}}^{\times}$, such that

$$
\tilde{\Psi} \cap c \widehat{\mathcal{R}} c^{-1}=\tilde{\Psi}\left(\mathcal{O}_{K}\right) .
$$

The group $\mathscr{B}^{\times}$acts on the set of such optimal embeddings by conjugation similar to (3.3), and $\operatorname{Pic}\left(\mathcal{O}_{K}\right)$ acts on the conjugacy classes of such embeddings.

Given $(\widetilde{\Psi}, c)$, let $\tau_{\widetilde{\Psi}} \in \mathbb{C}-\mathbb{R}$ be the fixed point under the action of $\widetilde{\Psi}\left(K^{\times}\right) \subset B^{\times}$, such that the induced action on the co-tangent line of $\mathbb{C}-\mathbb{R}$ is given by the character $z \rightarrow z / \bar{z}$. Let $P_{\widetilde{\Psi}} \in X_{\mathcal{B}}\left(\mathfrak{n}^{+}\right)(\mathbb{C})$ be the image of $\left(\tau_{\widetilde{\Psi}}, c\right)$ in the double coset (4.1). Then $P_{\widetilde{\Psi}}$ depends only on the conjugacy class of $(\widetilde{\Psi}, c)$. 
By the theory of complex multiplication one has $P_{\widetilde{\Psi}} \in X_{\mathcal{B}}\left(\mathfrak{n}^{+}\right)(H)$, where $H / K$ is the Hilbert class field of $K$. The point $P_{\widetilde{\Psi}}$ is called the CM point associated to $\widetilde{\Psi}$. We have the Shimura reciprocity law: identifying $\operatorname{Gal}(H / K) \cong \operatorname{Pic}\left(\mathcal{O}_{K}\right)$ via the Artin isomorphism (the convention being that a prime $q$ is mapped to the inverse of the Frobenius at $\mathfrak{q})$, one has for $\rho \in \operatorname{Pic}\left(\mathcal{O}_{K}\right)$,

$$
\left(P_{\widetilde{\Psi}}\right)^{\rho}=P_{\widetilde{\Psi}^{\rho}} .
$$

The moduli interpretation of Heegner points is as follows ([40], Section 2). First some notations. For any $M$-scheme $S$, and $[A, C] \in \mathcal{F}(S)$, denote by $\operatorname{End}_{\mathcal{F}}([A, C])$ the $\mathcal{O}_{M}$-subalgebra of $\operatorname{End}_{\mathcal{O}_{\mathcal{D}}}(A)$ generated by elements $\phi$, such that $\phi \phi^{*} \in F^{\times}$ (recall that $\phi \rightarrow \phi^{*}$ is the Rosati involution defined by a polarization from the data $\mathcal{F}(S)$ ), and such that $\phi(C) \subset C$. Also denote $K^{\prime}:=K \otimes_{F} M$.

Now let $x \in X_{\mathscr{B}}\left(\mathfrak{n}^{+}\right)(\mathbb{C})$. The image of $x$ in $X_{\mathfrak{D}}\left(\mathfrak{n}^{+}\right)(\mathbb{C})$ can be represented by $[A, C] \in \mathscr{F}(\mathbb{C})$. Then $x$ is a Heegner point if and only if there is an isomorphism

$$
\alpha: K^{\prime} \cong \operatorname{End}_{\mathcal{F}}([A, C]) \otimes \mathbb{Q}
$$

over $M$, such that

$$
\mathcal{O}_{K}=\left\{a \in K: \alpha(a) \in \operatorname{End}_{\mathcal{F}}([A, C])\right\} .
$$

4.3. $p$-adic description. We now give a $p$-adic description of Heegner points parallel to the previous section. This is based on the theorem of Cerednik-Drinfeld [6], [7], [33].

First recall that $\mathscr{B}_{v} \cong B_{v}$ for any place $v \neq v_{0}, \mathfrak{p}$. We have chosen Eichler orders $\mathcal{R}$ and $R$ of level $\mathfrak{n}^{+}$, of $\mathcal{B}$ and $B$ respectively. Assume that for primes $\mathfrak{I} \neq \mathfrak{p}$, the isomorphism $\mathscr{B}_{\mathfrak{I}} \cong B_{\mathfrak{I}}$ is chosen so that the local Eichler order $\mathcal{R}_{\mathfrak{I}}=\mathcal{R} \otimes_{\mathcal{O}_{F}} \mathcal{O}_{F_{\mathfrak{I}}}$ of $B_{\mathfrak{I}}$ of level $\mathfrak{n}^{+}$is mapped onto the local Eichler order $R_{\mathfrak{I}}=R \otimes_{\mathcal{O}_{F}} \mathcal{O}_{F_{\mathfrak{I}}}$ of $B_{\mathfrak{l}}$ of level $\mathfrak{n}^{+}$.

Let $X_{\mathcal{B}}\left(\mathfrak{n}^{+}\right)\left(\mathbb{C}_{p}\right)^{\text {an }}$ be the rigid analytic space associated to $X_{\mathcal{B}}\left(\mathfrak{n}^{+}\right)\left(\mathbb{C}_{p}\right)$. The theorem of Cerednik-Drinfeld gives the $p$-adic analogue of the uniformization (4.2), with $\mathfrak{h}$ replaced by the $p$-adic upper half plane $\mathscr{H}$ over $F_{\mathfrak{p}}$.

Recall the subgroup $\Gamma_{i}=\Gamma_{i}\left(\mathfrak{n}^{+}, \mathfrak{n}^{-}\right) \subset B^{\times}$defined as in (2.13). As before identify $\Gamma_{i}$ as a discrete subgroup of $B_{\mathfrak{p}}^{\times} \cong \mathrm{GL}_{2}\left(F_{\mathfrak{p}}\right)$. The result of CerednikDrinfeld states (see Theorem 3.1 of [7] or Theorem 5.3 of [33])

$$
\bigsqcup_{i=1}^{h} \Gamma_{i} \backslash \mathcal{H}\left(\mathbb{C}_{p}\right) \cong X_{\mathcal{B}}\left(\mathfrak{n}^{+}\right)\left(\mathbb{C}_{p}\right)^{\mathrm{an}} .
$$

Furthermore, the isomorphism is defined over $\mathbb{Q}_{p^{2 d}}$, i.e., equivariant with respect to the action of $\operatorname{Gal}\left(\overline{\mathbb{Q}}_{p} / \mathbb{Q}_{p^{2 d}}\right)$ on both sides. There is a corresponding result for 
$X_{\mathfrak{D}}\left(\mathfrak{n}^{+}\right)\left(\mathbb{C}_{p}\right)^{\text {an }}$, which is the $p$-adic analogue of (4.3). In fact, in the approach of [6], [7] the $p$-adic uniformization for $X_{\mathcal{B}}\left(\mathfrak{n}^{+}\right)\left(\mathbb{C}_{p}\right)^{\text {an }}$ is deduced from that of $X_{\mathfrak{D}}\left(\mathfrak{n}^{+}\right)\left(\mathbb{C}_{p}\right)^{\text {an }}$.

Using this description, we can relate the optimal embeddings of Section 3.2 to Heegner points. We use the description as in Section 3.4. Thus let $[\Psi]=[\Psi, g]_{i} \in$ $\operatorname{Emb}_{F}\left(K, B, \mathfrak{n}^{+}\right)$be a conjugacy class of optimal embeddings of $K$ to $B$ of level $\mathfrak{n}^{+}$, identified as an element of the $i$-th component of (3.11). As in (3.6), let $\tau_{\Psi}, \bar{\tau}_{\Psi} \in$ $\Gamma_{i} \backslash \mathscr{H}^{\prime}\left(\mathbb{Q}_{p^{2 d}}\right)=\widetilde{\Gamma}_{i} \backslash \mathcal{H}\left(\mathbb{Q}_{p^{2 d}}\right)$ be the fixed points under the action of $\Psi\left(K_{\mathfrak{p}}\right)$, such that $\tau_{\Psi}$ satisfies (3.7). Let $P_{\Psi} \in X_{\mathcal{B}}\left(\mathfrak{n}^{+}\right)\left(\mathbb{C}_{p}\right)^{\text {an }}$ be the corresponding point that lives on the $i$-th component of (4.7). Using the description of $p$-adic uniformization as in [6], [7], it can be shown that (see for example [3], Section 5) the image of $P_{\Psi}$ in $X_{\mathfrak{D}}\left(\mathfrak{n}^{+}\right)\left(\mathbb{C}_{p}\right)^{\text {an }}$ satisfies (4.5) and (4.6). Thus $P_{\Psi}$ is a Heegner point, in particular, $P_{\Psi} \in X_{\mathcal{B}}\left(\mathfrak{n}^{+}\right)(H)$, and we have the analogue of (4.4): for any $\rho \in \operatorname{Pic}\left(\mathcal{O}_{K}\right) \cong$ $\operatorname{Gal}(H / K)$,

$$
\left(P_{\Psi}\right)^{\rho}=P_{\Psi^{\rho}}
$$

4.4. Heegner points on elliptic curves. We refer back to Section 2.11. Thus $E / F$ is a modular elliptic curve, corresponding to a weight two cuspidal Hilbert eigenform $\mathbf{f}_{E}$, of conductor $\mathfrak{n}=\mathfrak{p} \mathfrak{n}^{+} \mathfrak{n}^{-}$. By the Jacquet-Langlands correspondence applied to $\mathscr{B}$, the cuspidal Hilbert eigenform $\mathbf{f}_{E}$, and hence $E / F$, is associated to a quaternionic eigenform on $\mathcal{B}^{\times}$. Geometrically this give rise to parametrization of $E$ by the Shimura curve $X_{\mathcal{B}}\left(\mathfrak{n}^{+}\right)$.

More precisely, let $\operatorname{Pic}^{0}\left(X_{\mathscr{B}}\left(\mathfrak{n}^{+}\right)\right) / F$ be the Picard variety of $X_{\mathcal{B}}\left(\mathfrak{n}^{+}\right) / F$. Note that, since $X_{\mathscr{B}} / F$ is not geometrically connected, $\operatorname{Pic}^{0}\left(X_{\mathcal{B}}\left(\mathfrak{n}^{+}\right)\right)$parametrizes divisors of degree zero on each geometric component, modulo the principal divisors. Over $\mathbb{C}$, we have

$$
\operatorname{Pic}^{0}\left(X_{\mathcal{B}}\left(\mathfrak{n}^{+}\right)\right)(\mathbb{C})=\prod_{i=1}^{h} \operatorname{Jac}\left(\Delta_{i} \backslash \mathfrak{h}\right) .
$$

The quaternionic eigenform on $\mathscr{B}^{\times}$corresponding to $\mathbf{f}_{E}$ then gives rise to the parametrization $\varphi_{E}$ defined over $F$,

$$
\varphi_{E}: \operatorname{Pic}^{0}\left(X_{\mathcal{B}}\left(\mathfrak{n}^{+}\right)\right) \rightarrow E,
$$

which is equivariant with respect to the Hecke operators $T_{\mathfrak{I}}$ for $\mathfrak{l} \nmid \mathfrak{n}$ (here $T_{\mathfrak{I}}$ acts on $E$ by multiplication by $\left.C\left(\mathfrak{l}, \mathbf{f}_{E}\right) \in \mathbb{Z}\right)$.

Let $P_{\Psi}$ be the CM point attached to the class of an optimal embedding of $K$ to $B$, and let $\psi_{K}$ be as in Section 3.1 a genus character of $K$, with $K^{\psi_{K}}$ the genus field 
(i.e., the extension of $K$ cut out by $\psi_{K}$ ). As in the situation of Proposition 3.16, put

$$
P_{\psi_{K}}=\sum_{\rho \in \operatorname{Pic}\left(\mathcal{O}_{K}\right)} \psi_{K}(\rho)\left(P_{\Psi}\right)^{\rho}=\sum_{\rho \in \operatorname{Pic}\left(\mathcal{O}_{K}\right)} \psi_{K}(\rho)\left(P_{\Psi^{\rho}}\right) .
$$

Then $P_{\psi_{K}}$ is a divisor on $X_{\mathcal{B}}\left(\mathfrak{n}^{+}\right)$.

In order to use the parametrization $\varphi_{E}$ of (4.8) to construct Heegner points on $E$, we need to modify $P_{\psi_{K}}$ to obtain a divisor of degree zero on each geometric component of $X_{\mathcal{B}}\left(\mathfrak{n}^{+}\right)$.

Let $\xi \in \operatorname{Pic}\left(X_{\mathscr{B}}\right)(F) \otimes \mathbb{Q}$ be the Hodge class [40]; the class $\xi$ has degree one on each geometric component, and satisfies the relation

$$
T_{\mathfrak{l}} \xi=(\mathcal{N} \mathfrak{l}+1) \xi
$$

for $\mathfrak{l}$ prime to $\mathfrak{n}$. Let $\xi_{i}$ be the $i$-th component of $\xi$.

Suppose that $i_{\Psi^{\rho}}$ is the index of the component of $X_{\mathcal{B}}\left(\mathfrak{n}^{+}\right)$on which $P_{\Psi^{\rho}}$ lies. Define $\widetilde{P}_{\psi_{K}} \in \operatorname{Pic}^{0}\left(X_{\mathcal{B}}\left(\mathfrak{n}^{+}\right)\right) \otimes \mathbb{Q}$ as

$$
\widetilde{P}_{\psi_{K}}=\text { class of } \sum_{\rho \in \operatorname{Pic}\left(\mathcal{O}_{K}\right)} \psi_{K}(\rho)\left(P_{\Psi^{\rho}}-\xi_{i_{\Psi^{\rho}}}\right) .
$$

We have $\widetilde{P}_{\psi_{K}} \in\left(\operatorname{Pic}^{0}\left(X_{\mathcal{B}}\right)\left(K^{\psi_{K}}\right) \otimes \mathbb{Q}\right)_{\psi_{K}}$. Define

$$
\mathbf{P}_{\psi_{K}}=\varphi_{E}\left(\widetilde{P}_{\psi_{K}}\right) \in\left(E\left(K^{\psi_{K}}\right) \otimes \mathbb{Q}\right)_{\psi_{K}},
$$

called the Heegner point attached to $\Psi$ and $\psi_{K}$.

We need the following result which follows from Zhang's generalization of the Gross-Zagier formula, and the work of Kolyvagin-Logachev:

Theorem 4.1. [40], [24] $\mathbf{P}_{\psi_{K}}$ is of infinite order if and only if $L^{\prime}\left(1, E / K, \psi_{K}\right) \neq 0$, in which case $\operatorname{dim}\left(E\left(K^{\psi_{K}}\right) \otimes \mathbb{Q}\right)_{\psi_{K}}=1$.

We can compute $\mathbf{P}_{\psi_{K}}$ in another way. For any prime $\mathfrak{l}$ of $\mathcal{O}_{F}$, denote by $C_{\mathfrak{l}}=$ $C\left(\mathfrak{l}, \mathbf{f}_{E}\right)$ the normalized Fourier coefficient of $\mathbf{f}_{E}$ at $\mathfrak{l}$. Choose $\mathfrak{l}$ to be relatively prime to $\mathfrak{n}$, and whose class in $\mathrm{Cl}_{F}$ is trivial (such $\mathfrak{l}$ exists by Cebotarev density theorem). For such an $\mathfrak{l}$, the action of the Hecke correspondence $T_{\mathfrak{l}}$ on $X_{\mathcal{B}}\left(\mathfrak{n}^{+}\right)$preserves the geometric components, and is of degree $\mathcal{N} \mathfrak{l}+1$ on each component. In particular, it follows from (4.9) that

$$
T_{\mathfrak{l}} \xi_{i}=(\mathcal{N} \mathfrak{I}+1) \xi_{i} \quad \text { for each } i .
$$

Then by the Hecke equivariance property of the Shimura curve parametrization $\varphi_{E}$ and (4.10), we have (here $C_{\mathfrak{l}}=C\left(\mathfrak{l}, \mathbf{f}_{E}\right)$ )

$$
\left(\mathcal{N} \mathfrak{l}+1-C_{\mathfrak{l}}\right) \mathbf{P}_{\psi_{K}}=\varphi_{E}\left(\left(\mathcal{N} \mathfrak{l}+1-T_{\mathfrak{l}}\right) \widetilde{P}_{\psi_{K}}\right)=\varphi_{E}\left(\left(\mathcal{N} \mathfrak{l}+1-T_{\mathfrak{l}}\right) P_{\psi_{K}}\right) .
$$


Hence fix such an $\mathfrak{l}$, and set

$$
Q_{\psi_{K}}=\left(\mathcal{N} \mathfrak{I}+1-C_{\mathfrak{l}}\right)^{-1}\left(\mathcal{N} \mathfrak{I}+1-T_{\mathfrak{l}}\right) P_{\psi_{K}} .
$$

Then $Q_{\psi_{K}} \in \operatorname{Div}^{0}\left(X_{\mathcal{B}}\left(\mathfrak{n}^{+}\right)\right) \otimes \mathbb{Q}$, whose image in $E$ under $\varphi_{E}$ is $\mathbf{P}_{\psi_{K}}$.

Now suppose that the genus character $\psi_{K}$ corresponds to the pair of quadratic Hecke characters $\psi_{1}, \psi_{2}$ of $F$, so $K^{\psi_{K}}=F^{\psi_{1}, \psi_{2}}$. Assume that

$$
L\left(1, E / F, \psi_{1}\right)=0, \quad L\left(1, E / F, \psi_{2}\right) \neq 0 .
$$

Using (3.2) it follows that

$$
L^{\prime}\left(1, E / F, \psi_{K}\right)=L^{\prime}\left(1, E / F, \psi_{1}\right) \cdot L\left(1, E / F, \psi_{2}\right),
$$

and hence it follows from Theorem 4.1 that the point $\mathbf{P}_{\psi_{K}}$ is of infinite order if and only if $L^{\prime}\left(1, E / F, \psi_{1}\right) \neq 0$.

By the results of Kolyvagin-Logachev [24], the assumption that $L\left(1, E / F, \psi_{2}\right) \neq$ 0 gives

$$
\operatorname{dim}\left(E\left(F^{\psi_{2}}\right) \otimes \mathbb{Q}\right)_{\psi_{2}}=0
$$

Corollary 4.2. The element $\mathbf{P}_{\psi_{K}}$ in $\left(E\left(F^{\psi_{1}, \psi_{2}}\right) \otimes \mathbb{Q}\right)_{\psi_{K}}$ lies in $\left(E\left(F^{\psi_{1}}\right) \otimes \mathbb{Q}\right)_{\psi_{1}}$.

Proof. First note that, in the notation of Section 3.1,

$$
\mathbf{P}_{\psi_{K}}+t_{1} \cdot \mathbf{P}_{\psi_{K}}
$$

lies in

$$
\left(E\left(F^{\psi_{1}, \psi_{2}}\right) \otimes \mathbb{Q}\right)_{\psi_{K}} \cap\left(E\left(F^{\psi_{2}}\right) \otimes \mathbb{Q}=\left(E\left(F^{\psi_{2}}\right) \otimes \mathbb{Q}\right) \psi_{2}\right.
$$

hence is zero modulo torsion by (4.13). On the other hand, since $\mathbf{P}_{\psi_{K}} \in\left(E\left(F^{\psi_{1}, \psi_{2}}\right) \otimes\right.$ Q) $\psi_{K}$, we have

$$
t_{1} t_{2} \cdot \mathbf{P}_{\psi_{K}}=-\mathbf{P}_{\psi_{K}}
$$

hence

$$
t_{2} \cdot \mathbf{P}_{\psi_{K}}=-t_{1} \cdot \mathbf{P}_{\psi_{K}} .
$$

So modulo torsion $t_{2} \cdot \mathbf{P}_{\psi_{K}}=\mathbf{P}_{\psi_{K}}$, and so

$$
\mathbf{P}_{\psi_{K}}=\frac{1}{2}\left(\mathbf{P}_{\psi_{K}}+t_{2} \cdot \mathbf{P}_{\psi_{K}}\right) \in\left(E\left(F^{\psi_{1}}\right) \otimes \mathbb{Q}\right)_{\psi_{1}} .
$$

Corollary 4.3. Let Frob $_{\mathfrak{p}}$ be the Frobenius element at $\mathfrak{p}$ of the extension $F^{\psi_{1}} / F$. Then

$$
\operatorname{Frob}_{\mathfrak{p}} \mathbf{P}_{\psi_{K}}=\psi_{1}(\mathfrak{p}) \mathbf{P}_{\psi_{K}}
$$


4.5. Heegner points and $p$-adic periods. We can now relate the $p$-adic periods of Section 2.9 with Heegner points. We maintain the notations of Section 4.4, and assume (4.12) holds.

Fist we give a $p$-adic analytic description of the Shimura curve parametrization (4.8).

We refer to the notations of Section 2.11. Replacing the quaternionic form $\Phi$ on $B^{\times}$by a suitable integer multiple of $\Phi$, we may assume that the lattice of periods defined by $\omega_{\phi^{i}}$ is contained in the lattice of Tate periods of $E / F_{\mathfrak{p}}$.

Choose a Tate uniformization isomorphism

$$
\Theta_{E / F_{\mathfrak{p}}}: \mathbb{C}_{p}^{\times} / Q_{E}^{\mathbb{Z}} \stackrel{\sim}{\longrightarrow} E\left(\mathbb{C}_{p}\right) .
$$

Then over $\mathbb{C}_{p}$, the Shimura curve parametrization

$$
\varphi_{E}: \operatorname{Pic}^{0}\left(X_{\mathcal{B}}\left(\mathfrak{n}^{+}\right)\right)\left(\mathbb{C}_{p}\right)=\prod_{i} \operatorname{Jac}\left(\Gamma_{i} \backslash \mathcal{H}\left(\mathbb{C}_{p}\right)\right) \rightarrow E\left(\mathbb{C}_{p}\right)
$$

can be described as follows. Let

$$
D=\sum_{i=1}^{h} D_{i} \in \operatorname{Div}^{0}\left(X_{\mathcal{B}}\left(\mathfrak{n}^{+}\right)\left(\mathbb{C}_{p}\right)=\bigoplus_{i=1}^{h} \operatorname{Div}^{0}\left(\Gamma_{i} \backslash \mathscr{H}\left(\mathbb{C}_{p}\right)\right),\right.
$$

with $D_{i} \in \operatorname{Div}^{0}\left(\Gamma_{i} \backslash \mathscr{H}\left(\mathbb{C}_{p}\right)\right)$. Then for each component we have by [13], Theorem 2.32 (which is a reformulation of a theorem of Manin-Drinfeld) that

$$
\varphi_{E}\left(D_{i}\right)=\Theta_{E / F_{\mathfrak{p}}}\left(\oiint_{D_{i}} \omega_{\phi_{i}}\right) \text {. }
$$

So by additivity,

$$
\varphi_{E}(D)=\sum_{i=1}^{h} \Theta_{E / F_{\mathfrak{p}}}\left(\S_{D_{i}} \omega_{\phi_{i}}\right)=\Theta_{E / F_{\mathfrak{p}}}\left(\S_{D} \omega_{\Phi}\right)
$$

(here $\sum$ is the addition on $E$ ).

Given the Tate uniformization, define the maps

$$
\begin{aligned}
\log _{E / F_{\mathfrak{p}}}: E\left(\mathbb{Q}_{p^{2 d}}\right)_{\alpha_{\mathfrak{p}}} \rightarrow F_{\mathfrak{p}}, \\
\operatorname{logNorm}_{E / F_{\mathfrak{p}}}: E\left(\mathbb{Q}_{p^{2 d}}\right)_{\alpha_{\mathfrak{p}}} \rightarrow \mathbb{Q}_{p}
\end{aligned}
$$

as the composition of the following maps:

$$
\begin{aligned}
& \log _{E / F_{\mathfrak{p}}}: E\left(\mathbb{Q}_{p^{2 d}}\right)_{\alpha_{\mathfrak{p}}} \stackrel{\Theta_{E / F_{\mathfrak{p}}}^{-1}}{\longrightarrow} F_{\mathfrak{p}}^{\times} / Q_{E}^{\mathbb{Z}} \stackrel{\log _{Q_{E}}}{\longrightarrow} F_{\mathfrak{p}}, \\
& \log _{\text {Norm }} E / F_{\mathfrak{p}}: E\left(\mathbb{Q}_{p} 2 d\right)_{\alpha_{\mathfrak{p}}} \stackrel{\Theta_{E / F_{\mathfrak{p}}}^{-1}}{\longrightarrow} F_{\mathfrak{p}}^{\times} / Q_{E}^{\mathbb{Z}} \stackrel{\mathcal{N}_{F_{\mathfrak{p}} / \mathbb{Q} p}}{\longrightarrow} \mathbb{Q}_{p}^{\times} / q_{E}^{\mathbb{Z}} \stackrel{\log _{q_{E}}}{\longrightarrow} \mathbb{Q}_{p}
\end{aligned}
$$


(recall that $q_{E}=\mathcal{N}_{F_{\mathfrak{p}} / \mathbb{Q}_{p}} Q_{E}$ ). The maps $\log _{E / F_{\mathfrak{p}}}, \log \operatorname{Norm}_{E / F_{\mathfrak{p}}}$ are homomorphisms (with respect to the additive structure). Note that the kernel of $\log _{E / F_{\mathfrak{p}}}$ consists exactly of the torsion elements, but this is not the case for $\operatorname{logNorm}_{E / F_{\mathfrak{p}}}$. We extend the definition of $\log _{E / F_{\mathfrak{p}}}$ and $\operatorname{logNorm}_{E / F_{\mathfrak{p}}}$ to $E\left(\mathbb{Q}_{p^{2 d}}\right)_{\alpha_{\mathfrak{p}}} \otimes \mathbb{Q}$ by linearity.

Applying Corollary 2.28 to the divisor $Q_{\psi_{K}}$ of (4.11), we have

$$
I_{\Phi}\left(Q_{\psi_{K}}\right)=\log _{q_{E}} \mathcal{N}_{\mathbb{Q}_{p 2 d} / \mathbb{Q}_{p}}\left(f_{Q_{\psi_{K}}} \omega_{\Phi}\right) .
$$

The right hand side of (4.15) can be written as

$$
\begin{aligned}
\log _{q_{E}} \mathcal{N}_{\mathbb{Q}_{p} 2 d} / \mathbb{Q}_{p}\left(f_{Q_{\psi_{K}}} \omega_{\Phi}\right) & =\log _{q_{E}} \mathcal{N}_{F_{\mathfrak{p}} / \mathbb{Q}_{p}} \mathcal{N}_{\mathbb{Q}_{p^{2 d}} / F_{\mathfrak{p}}}\left(f_{Q_{\psi_{K}}} \omega_{\Phi}\right) \\
& =\log \operatorname{Norm}_{E / F_{\mathfrak{p}}} \Theta_{E / F_{\mathfrak{p}}} \mathcal{N}_{\mathbb{Q}_{p^{2 d}} / F_{\mathfrak{p}}}\left(f_{Q_{\psi_{K}}} \omega_{\Phi}\right) .
\end{aligned}
$$

Now as in (2.25), let $s$ be the non-trivial element of $\operatorname{Gal}\left(\mathbb{Q}_{p^{2 d}} / F_{\mathfrak{p}}\right)$. Then

$$
\begin{aligned}
\Theta_{E / F_{\mathfrak{p}}} \mathcal{N}_{\mathbb{Q}_{p^{2 d}} / F_{\mathfrak{p}}}\left(f_{Q_{\psi_{K}}} \omega_{\Phi}\right) & =\Theta_{E / F_{\mathfrak{p}}}\left(f_{Q_{\psi_{K}}} \omega_{\Phi}\right)+\Theta_{E / F_{\mathfrak{p}}}\left(s \cdot f_{Q_{\psi_{K}}} \omega_{\Phi}\right) \\
& =\Theta_{E / F_{\mathfrak{p}}}\left(f_{Q_{\psi_{K}}} \omega_{\Phi}\right)+\alpha_{\mathfrak{p}} s \cdot \Theta_{E / F_{\mathfrak{p}}}\left(\oint_{Q_{\psi_{K}}} \omega_{\Phi}\right) .
\end{aligned}
$$

Now by (4.14)

$$
\Theta_{E / F_{\mathfrak{p}}}\left(f_{Q_{\psi_{K}}} \omega_{\Phi}\right)=\varphi_{E}\left(Q_{\psi_{K}}\right)=\mathbf{P}_{\psi_{K}}
$$

and by Corollary 4.3,

$$
s \cdot \mathbf{P}_{\psi_{K}}=\psi_{1}(\mathfrak{p}) \mathbf{P}_{\psi_{K}} \cdot
$$

Hence

$$
I_{\Phi}\left(Q_{\psi_{K}}\right)=\log _{q_{E}} \mathcal{N}_{\mathbb{Q}_{p} 2 d} / \mathbb{Q}_{p}\left(f_{Q_{\psi_{K}}} \omega_{\Phi}\right)=\left(1+\alpha_{\mathfrak{p}} \psi_{1}(\mathfrak{p})\right) \operatorname{logNorm}_{E / F_{\mathfrak{p}}} \mathbf{P}_{\psi_{K}}
$$

Now we need a

Lemma 4.4. With respect to the choice of $\mathfrak{l}$ as in (4.10), we have for any divisor $D \in \operatorname{Div}\left(X_{\mathcal{B}}\left(\mathfrak{n}^{+}\right)\left(\mathbb{C}_{p}\right)\right)=\bigoplus_{i=1}^{h} \operatorname{Div}\left(\Gamma_{i} \backslash \mathscr{H}\left(\mathbb{C}_{p}\right)\right)$ the equality

$$
I_{\Phi}\left(T_{\mathfrak{l}} D\right)=C_{\mathfrak{l}} I_{\Phi}(D) .
$$

Proof. As $\Upsilon$ is trivial in $\mathrm{Cl}_{F}$, it preserves the components $\Gamma_{i} \backslash \mathscr{H}\left(\mathbb{C}_{p}\right)$ of $X_{\mathcal{B}}\left(\mathfrak{n}^{+}\right)\left(\mathbb{C}_{p}\right)$, so by linearity, it suffice to show that for any $\tau \in \Gamma_{i} \backslash \mathscr{H}\left(\mathbb{C}_{p}\right)$,

$$
I_{\phi^{i}}\left(T_{\Upsilon} \tau\right)=C_{\Upsilon} I_{\phi^{i}}(\tau)
$$

whose proof is the same as Proposition 2.18 of [4]. 
From this lemma, we have from the definition of $Q_{\psi_{K}}$ as in (4.11) that

$$
I_{\Phi}\left(Q_{\psi_{K}}\right)=I_{\Phi}\left(P_{\psi_{K}}\right)
$$

On the other hand, by Proposition 3.16, we have

$$
\left.\frac{d}{d k} \mathscr{L}_{p}\left(\mathbf{f}_{\infty} / K, \psi_{K}, k\right)\right|_{k=2}=\frac{1}{2} I_{\Phi}\left(P_{\psi_{K}}\right)
$$

We summarize the discussion as the following proposition (here the point $\mathbf{P}_{\psi_{K}}$ is renamed as $\left.\mathbf{P}_{\psi_{1}}\right)$ :

Proposition 4.5. Assume that $L\left(1, E / F, \psi_{1}\right)=0, L\left(1, E / F, \psi_{2}\right) \neq 0$. Then there is a global point $\mathbf{P}_{\psi_{1}} \in\left(E\left(F^{\psi_{1}}\right) \otimes \mathbb{Q}\right)_{\psi_{1}}$ such that

$$
\left.\frac{d}{d k} \mathscr{L}_{p}\left(\mathbf{f}_{\infty} / K, \psi_{K}, k\right)\right|_{k=2}=\frac{1+\alpha_{\mathfrak{p}} \psi_{1}(\mathfrak{p})}{2} \log \operatorname{Norm}_{E / F_{\mathfrak{p}}} \mathbf{P}_{\psi_{1}} .
$$

Furthermore, the point $\mathbf{P}_{\psi_{1}}$ is of infinite order if and only if $L^{\prime}\left(1, E / F, \psi_{1}\right) \neq 0$.

\section{Main results}

5.1. $p$-adic $\boldsymbol{L}$-functions of Hilbert modular forms. In this section, we recall some results from [26] about $p$-adic $L$-functions of Hilbert modular forms.

First we need some notations. Let $\psi=\otimes_{\nu} \psi_{\nu}$ be a Hecke character of $F$ of finite order. Denote by $\operatorname{sig}(\psi) \in\{ \pm 1\}^{d}$, the signature of $\psi$, as the $d$-tuple $\left(\psi_{\nu}(-1)\right)_{v \mid \infty}$. Thus $\operatorname{sig}(\psi)=(1, \ldots, 1)$ if $\psi$ is unramified at all the infinite places. As another example, for $\omega_{F}$ the Teichmüller character of $F$, one has $\operatorname{sig}\left(\omega_{F}\right)=(-1, \ldots,-1)$.

In general, for $w=\left(w_{\nu}\right)_{\nu \mid \infty} \in\{ \pm 1\}^{d}$, denote $\operatorname{sgn}(w):=\prod_{\nu \mid \infty} w_{\nu}$. We abbreviate $\operatorname{sgn}(\operatorname{sig}(\psi))$ as $\operatorname{sgn}(\psi)$. Also denote by $\tau(\psi)$ the Gauss sum of $\psi([31]$, equation 3.9).

Let $\mathbf{g}$ be a Hilbert newform of parallel weight $k \geq 2$. Fix an embedding $\overline{\mathbb{Q}} \hookrightarrow \mathbb{C}$. Recall Shimura's rationality result on $L$-values, cf. [31], Theorem 4.3 (I) and [11], Remark (ii) on p. 1027: for every $w \in\{ \pm 1\}^{d}$ one can choose $\Omega_{\mathbf{g}}^{w} \in \mathbb{C}^{\times}$such that, for an integer $0 \leq r \leq k-2$ and a finite order Hecke character $\psi$ of $F$ with conductor $c_{\psi}$, the expression

$$
L^{\mathrm{alg}}(r+1, \mathbf{g}, \psi):=\frac{D_{F}^{r}(r)^{d} \mathcal{N} c_{\psi}^{r+1}}{(-2 \pi i)^{d r} \tau\left(\psi^{-1}\right)} \frac{L(r+1, \mathbf{g}, \psi)}{\Omega_{\mathbf{g}}^{(-1)^{r} \operatorname{sig}(\psi)}}
$$

is an algebraic number, called the algebraic part of the $L$-value. Furthermore, for every $h \in \operatorname{Aut}(\overline{\mathbb{Q}} / \mathbb{Q}(\mathbf{g}))$,

$$
L^{\mathrm{alg}}(r+1, \mathbf{g}, \psi)^{h}=L^{\mathrm{alg}}\left(r+1, \mathbf{g}, \psi^{h}\right)
$$


which implies in particular that

$$
L^{\mathrm{alg}}(r+1, \mathbf{g}, \psi) \in \mathbb{Q}(\mathbf{g}, \psi),
$$

where $\mathbb{Q}(\mathbf{g}, \psi)$ is the number field generated by the (normalized) Fourier coefficients of $\mathbf{g}$ and the values of $\psi$. In particular, if the Fourier coefficients of $\mathbf{g}$ are rational integers, and $\psi$ is quadratic, then the values $L^{\mathrm{alg}}(r+1, \mathbf{g}, \psi)$ are rational numbers.

Thus the numbers $\Omega_{\mathbf{g}}^{w}$ are serving as "transcendental factors" of the special $L$ values. Notice that if $\widetilde{\Omega}_{\mathbf{g}}^{w} \in \mathbb{C}^{\times}$is another set of numbers such that

$$
\frac{\widetilde{\Omega}_{\mathbf{g}}^{w}}{\Omega_{\mathbf{g}}^{w}} \in \mathbb{Q}(\mathbf{g}) \quad \text { for each } w \in\{ \pm 1\}^{d},
$$

then one can equally define $L^{\text {alg }}(r+1, \mathbf{g}, \psi)$ with respect to $\widetilde{\Omega}_{\mathbf{g}}^{w}$ instead of $\Omega_{\mathbf{g}}^{w}$, and such that equation (5.2) still holds. In what follows, we always fix a choice of the numbers $\Omega_{\mathrm{g}}^{w}$ to define the algebraic part of $L$-values.

Now suppose that $\mathbf{g}$ is ordinary at $\mathfrak{p}$. Let $\chi$ be a finite order Hecke character of $F$ unramified outside $\mathfrak{p}$ and the infinite places, and $\psi$ a finite order Hecke character of $F$ unramified at the conductor of $\mathbf{g}$. In [11], Theorem 1 , the $p$-adic $L$-function $L_{p}(s, \mathbf{g}, \chi \psi)$ is constructed (with respect to a specific choice of $\Omega_{\mathbf{g}}^{w}$ ). It satisfies the interpolation property: for $0 \leq r \leq k-2$,

$$
\begin{aligned}
L_{p}(r+1, \mathbf{g}, \chi \psi)=( & \left.1-\frac{\chi \psi \omega_{F}^{-r}(\mathfrak{p}) \mathcal{N} \mathfrak{p}^{r}}{\alpha(\mathfrak{p}, \mathbf{g})}\right) \\
& \cdot\left(1-\frac{\left(\chi \psi \omega_{F}^{-r}\right)^{-1}(\mathfrak{p}) \beta(\mathfrak{p}, \mathbf{g})}{\mathcal{N} \mathfrak{p}^{r+1}}\right) L^{\mathrm{alg}}\left(r+1, \mathbf{g},\left(\chi \psi \omega_{F}^{-r}\right)^{-1}\right) .
\end{aligned}
$$

Here $\alpha(\mathfrak{p}, \mathbf{g})$ and $\beta(\mathfrak{p}, \mathbf{g})$ are the $p$-adic unit root and non-unit root of the Hecke polynomial

$$
X^{2}-C(\mathfrak{p}, \mathbf{g}) X+\epsilon_{\mathfrak{p}} \mathcal{N} \mathfrak{p}^{k-1}
$$

with $\epsilon_{\mathfrak{p}}=1$ if $\mathfrak{p}$ does not divide the conductor of $\mathbf{g}$, and equal to zero otherwise. We have the convention that $\chi \psi \omega_{F}^{-r}(\mathfrak{p})=0$ if $\chi \psi \omega_{F}^{-r}$ is ramified at $\mathfrak{p}$.

If $\chi, \psi$ are trivial, we denote the $p$-adic $L$-function as $L_{p}(s, \mathbf{g})$.

As in the previous sections, let $\mathbf{f}_{\infty}$ be the Hida family lifting $\mathbf{f}_{E}=\mathbf{f}_{2}$, with $E$ a modular elliptic curve over $F$, whose conductor $\mathfrak{n}$ can be written in the form $\mathfrak{p} \mathfrak{n}^{+} \mathfrak{n}^{-}$, with $\mathfrak{n}^{-}$satisfying condition 2.1. In Theorem 6.8 of [26], it was shown that the $p$-adic $L$-functions $L_{p}\left(s, \mathbf{f}_{k}^{\#}\right)$ attached to the weight $k$ specializations $\mathbf{f}_{k}$ of $\mathbf{f}_{\infty}$ for $k \in U \cap \mathbb{Z}^{\geq 2}$ ( $\mathbf{f}_{k}^{\#}$ is the newform whose $\mathfrak{p}$-stabilization is $\mathbf{f}_{k}$ ) can be interpolated to a two variable $p$-adic $L$-function, i.e., there exists a $p$-adic analytic function $L_{p}(s, k)=L_{p}\left(s, k, \mathbf{f}_{\infty}\right)$ of $p$-adic variables $s, k$ with $k$ taking values in 
a suitable $p$-adic neighbourhood $U$ of 2 , such that for $k \in U \cap \mathbb{Z}^{\geq 2}$, one has $L_{p}\left(s, k, \mathbf{f}_{\infty}\right)=L_{p}\left(s, \mathbf{f}_{k}^{\#}\right)$. In particular, $L_{p}(s, 2)=L_{p}\left(s, \mathbf{f}_{E}\right):=L_{p}(s, E / F)$ (let us note that one of the technicalities of [26] is to show that one can choose the numbers $\Omega_{\mathbf{f}_{k}^{\#}}^{w}$ appropriately so that this interpolation property holds).

As a special case of (5.3), take $\chi$ to be trivial, and $\psi$ quadratic, and $r=k / 2-1$. Then if $k \in U \cap \mathbb{Z}^{\geq 2}$ satisfies $k \equiv 2 \bmod 2(p-1)$, one has

$$
\begin{aligned}
L_{p}\left(k / 2, k, \mathbf{f}_{\infty}\right) & =L_{p}\left(k / 2, \mathbf{f}_{k}^{\#}, \psi\right) \\
& = \begin{cases}\left(1-\frac{\psi(\mathfrak{p}) \mathcal{N} \mathfrak{p}^{k / 2-1}}{\alpha\left(\mathfrak{p}, \mathbf{f}_{k}^{\#}\right)}\right)^{2} L^{\operatorname{alg}}\left(k / 2, \mathbf{f}_{k}^{\#}, \psi\right) & \text { if } k>2, \\
\left(1-\frac{\psi(\mathfrak{p})}{\alpha(\mathfrak{p}, E / F)}\right) L^{\mathrm{alg}}(1, E / F, \psi) & \text { if } k=2 .\end{cases}
\end{aligned}
$$

5.2. Proof of main results. We first need a fact about the functional equation of the complex $L$-function of $L(s, E / F)$. Let $\epsilon_{E / F} \in\{ \pm 1\}$ be the sign:

$$
L(2-s, E / F)=\epsilon_{E / F} L(s, E / F) .
$$

For any quadratic Hecke character $\psi$, whose conductor is relatively prime to that of $E / F$, the twisted $L$-function satisfies a similar functional equation:

$$
L(2-s, E / F, \psi)=\epsilon_{E / F}(\psi) L(s, E / F, \psi)
$$

with $\epsilon_{E / F}(\psi) \in\{ \pm 1\}$ given by the expression

$$
\epsilon_{E / F}(\psi)=\operatorname{sgn}(\psi) \psi(\mathfrak{n}) \cdot \epsilon_{E / F} .
$$

Proposition 5.1. Let $w \in\{ \pm 1\}^{d}$. In the case when $\mathfrak{n}^{+} \mathfrak{n}^{-}$is the square of an ideal (i.e., $\mathfrak{n}^{-}=\mathcal{O}_{F}$ and $\mathfrak{n}^{+}$is the square of an ideal), assume that the following condition holds:

$$
\alpha(\mathfrak{p}, E / F)=-\operatorname{sgn}(w) \epsilon_{E / F} .
$$

Then shrinking $U$ if necessary, there exists a p-adic analytic function $\eta_{w}$ defined on $U$ such that, for integers $k \in U \cap \mathbb{Z}^{\geq 2}$ with $k \equiv 2 \bmod 2(p-1)$, one has

$$
\eta_{w}(k)=\left\langle\Phi_{k}^{\#}, \Phi_{k}^{\#}\right\rangle i^{d} \frac{\Omega_{\mathbf{f}_{k}^{\#} \Omega_{\mathbf{f}_{k}^{\#}}^{-w}}}{\left\langle\mathbf{f}_{k}^{\#}, \mathbf{f}_{k}^{\#}\right\rangle}
$$

In fact, the proof below shows that the expression

$$
i^{d} \frac{\Omega_{\mathbf{f}_{k}^{\#}}^{w} \Omega_{\mathbf{f}_{k}^{\#}}^{-w}}{\left\langle\mathbf{f}_{k}^{\#}, \mathbf{f}_{k}^{\#}\right\rangle}
$$

lies in $\mathbb{Q}\left(\mathbf{f}_{k}^{\#}\right)^{\times}$. 
Proof. Let $S_{1}$ be the set of quadratic Hecke characters $\psi_{1}$ of $F$, unramified at the primes dividing $\mathfrak{n}$, and satisfying the following conditions:

(1) $\operatorname{sig}\left(\psi_{1}\right)=w$.

(2) $\psi_{1}(\mathfrak{p})=-\alpha(\mathfrak{p}, E / F)$.

(3) $\psi_{1}\left(\mathfrak{n}^{+} \mathfrak{n}^{-}\right)=-\alpha(\mathfrak{p}, E / F) \cdot \operatorname{sgn}(w) \cdot \epsilon_{E / F}$.

These conditions amount to specifying the local conditions for $\psi_{1}$ at the archimedean primes and at the primes dividing $\mathfrak{n}$. The set $S_{1}$ is non-empty. Indeed, the only point that needs to be addressed is condition (3), in the case when $\mathfrak{n}^{+} \mathfrak{n}^{-}$is the square of an ideal. In this case, since $\psi_{1}$ is quadratic, one has $\psi_{1}\left(\mathfrak{n}^{+} \mathfrak{n}^{-}\right)=1$, hence the reason for assuming (5.6) to hold.

For any $\psi_{1} \in S_{1}$, one has, using (5.5), that $\epsilon_{E / F}\left(\psi_{1}\right)=1$. By the main theorem of Friedberg-Hoffstein [15], we can pick a $\psi_{1} \in S_{1}$, such that $L\left(1, E / F, \psi_{1}\right) \neq 0$. Condition (2) implies that $L_{p}\left(s, E / F, \psi_{1}\right)$ does not have exceptional zero at $s=1$, so $L_{p}\left(1, E / F, \psi_{1}\right) \neq 0$.

Now let $S_{2}$ be the set of quadratic Hecke characters $\psi_{2}$ of $F$, with conductor relatively prime to that of $\psi_{1}$ and of $\mathfrak{n}$, satisfying the following conditions:

(1) $\operatorname{sig}\left(\psi_{2}\right)=-w$.

(2) $\psi_{2}(\mathfrak{p})=-\alpha(\mathfrak{p}, E / F)$.

(3) $\psi_{2}\left(\mathfrak{n}^{+} \mathfrak{n}^{-}\right)=(-1)^{d+1} \alpha(\mathfrak{p}, E / F) \cdot \operatorname{sgn}(w) \cdot \epsilon_{E / F}$.

Again $S_{2}$ is non-empty (notice that in the case when $\mathfrak{n}^{+} \mathfrak{n}^{-}$is a square, i.e., $\mathfrak{n}^{-}=\mathcal{O}_{F}$ and $\mathfrak{n}^{+}$is a square, one must have $d=[F: \mathbb{Q}]$ being even by condition 2.1 . Hence the last condition is again satisfied by (5.6)). For any $\psi_{2} \in S_{2}$, one has $\epsilon_{E / F}\left(\psi_{2}\right)=1$. By [15] again, we can pick a $\psi_{2} \in S_{2}$ so that $L\left(1, E / F, \psi_{2}\right) \neq 0$. Again, the conditions specified on $\psi_{2}$ ensures that $L_{p}\left(s, E / F, \psi_{2}\right)$ has no exceptional zero at $s=1$ and so $L_{p}\left(1, E / F, \phi_{2}\right) \neq 0$.

Hence shrinking $U$ if necessary, we may assume that the $p$-adic analytic functions $L_{p}\left(k / 2, k, \mathbf{f}_{\infty}, \psi_{1}\right)$ and $L_{p}\left(k / 2, k, \mathbf{f}_{\infty}, \psi_{2}\right)$ are non-zero on $U$.

Let $\zeta:=\psi_{1} \cdot \psi_{2}$. Then $\operatorname{sig}(\zeta)=(-1, \ldots,-1)$. Hence $\zeta$ cuts out a CM extension $K$ of $F$, in which $\mathfrak{p}$ splits (since $\left.\psi_{1} \psi_{2}(\mathfrak{p})=1\right)$, and the pair $\left(\psi_{1}, \psi_{2}\right)$ corresponds to a genus character $\psi_{K}$ of $K$.

Let $k \in U \cap \mathbb{Z}^{\geq 2}$ be an integer, with $k \equiv 2 \bmod 2(p-1)$. By equation (3.2) we have

$$
L\left(k / 2, \mathbf{f}_{k}^{\#} / K, \psi_{K}\right)=L\left(k / 2, \mathbf{f}_{k}^{\#}, \psi_{1}\right) \cdot L\left(k / 2, \mathbf{f}_{k}^{\#}, \psi_{2}\right)
$$

Hence $L\left(k / 2, \mathbf{f}_{k}^{\#} / K, \psi\right) \neq 0$ for $k$ specified as above. By Corollary 3.7, the value $L\left(k / 2, \mathbf{f}_{k}^{\#} / K, \psi\right)$ lies in $\mathbb{Q}\left(\mathbf{f}_{k}^{\#}\right)$. 
Using (5.1), we have, for $j=1,2$ (noting that $k / 2-1$ is even, as $p$ is odd):

$$
L^{\mathrm{alg}}\left(k / 2, \mathbf{f}_{k}^{\#}, \psi_{j}\right)=\frac{D_{F}^{k / 2-1}(k / 2-1) !^{d} \mathcal{N} c_{\psi_{j}}^{k / 2}}{(2 \pi i)^{d(k / 2-1)} \tau\left(\psi_{j}\right)} \frac{L\left(k / 2, \mathbf{f}_{k}^{\#}, \psi_{j}\right)}{\Omega_{\mathbf{f}_{k}^{\#}}^{\operatorname{sig}\left(\psi_{j}\right)}} \in \mathbb{Q}\left(\mathbf{f}_{k}^{\#}\right) .
$$

Now by Proposition 7.1 of Appendix I, we have the following conductors and Gauss sums identities:

$$
\begin{gathered}
D_{K} D_{F}^{-2}=\mathcal{N}_{F / \mathbb{Q}} D_{K / F}=\mathcal{N}_{F / \mathbb{Q}} \mathfrak{c}_{\psi_{1}} \mathcal{N}_{F / \mathbb{Q}} \mathfrak{c}_{\psi_{2}} \\
\tau\left(\psi_{1}\right) \tau\left(\psi_{2}\right)=i d \sqrt{\mathcal{N}_{F / \mathbb{Q}} \mathfrak{c}_{\psi_{1}} \mathcal{N}_{F / \mathbb{Q}} \mathfrak{c}_{\psi_{2}}} .
\end{gathered}
$$

Putting together (3.9), and (5.8)-(5.10), we obtain

$$
\begin{aligned}
& L^{\operatorname{alg}}\left(k / 2, \mathbf{f}_{k}^{\#} / K, \psi_{K}\right) \\
& \quad=\frac{u_{K}^{2}}{D_{K}^{k / 2-1}} i^{d} \frac{\Omega_{\mathbf{f}_{k}^{\#}}^{w} \Omega_{\mathbf{f}_{k}^{\#}}^{-w}}{\left\langle\mathbf{f}_{k}^{\#}, \mathbf{f}_{k}^{\#}\right\rangle} L^{\operatorname{alg}}\left(k / 2, \mathbf{f}_{k}^{\#}, \psi_{1}\right) \cdot L^{\operatorname{alg}}\left(k / 2, \mathbf{f}_{k}^{\#}, \psi_{2}\right) .
\end{aligned}
$$

Equation (5.11) implies that $i d \frac{\Omega_{\mathbf{f}_{k}^{\#}}^{w} \Omega_{\mathbf{f}_{k}^{*}}^{-w}}{\left\langle\mathbf{f}_{k}^{\#}, \mathbf{f}_{k}^{\#}\right\rangle} \in \mathbb{Q}\left(\mathbf{f}_{k}^{\#}\right)$.

Using equation (3.14), (3.15), and (5.4) we obtain

$$
\begin{aligned}
& \mathscr{L}_{p}\left(k / 2, \mathbf{f}_{\infty} / K, \psi_{K}\right)^{2}=\frac{u_{K}^{2}}{D_{K}^{k / 2-1}} \alpha(\mathfrak{p}, k)^{2}\left\langle\Phi_{k}^{\#}, \Phi_{k}^{\#}\right\rangle \\
& i^{d} \frac{\Omega_{\mathbf{f}_{k}^{\#}}^{w} \Omega_{\mathbf{f}_{k}^{\#}}^{-w}}{\left\langle\mathbf{f}_{k}^{\#}, \mathbf{f}_{k}^{\#}\right\rangle} L_{p}\left(k / 2, \mathbf{f}_{k}^{\#}, \psi_{1}\right) L_{p}\left(k / 2, \mathbf{f}_{k}^{\#}, \psi_{2}\right) \\
&=\frac{u_{K}^{2}}{\left\langle D_{K}\right\rangle^{k / 2-1}} \alpha(\mathfrak{p}, k)^{2}\left\langle\Phi_{k}^{\#}, \Phi_{k}^{\#}\right\rangle \\
& i^{d} \frac{\Omega_{\mathbf{f}_{k}^{\#}}^{w} \Omega_{\mathbf{f}_{k}^{\#}}^{-w}}{\left\langle\mathbf{f}_{k}^{\#}, \mathbf{f}_{k}^{\#}\right\rangle} L_{p}\left(k / 2, k, \mathbf{f}_{\infty}, \psi_{1}\right) L_{p}\left(k / 2, k, \mathbf{f}_{\infty}, \psi_{2}\right)
\end{aligned}
$$

(for the last equality, note that since $k / 2-1 \equiv 0 \bmod p-1$, one has $D_{K}^{k / 2-1}=$ $\left.\left\langle D_{K}\right\rangle^{k / 2-1}\right)$. Hence the proposition is proved, by defining

$$
\eta_{w}(k):=\frac{\left\langle D_{K}\right\rangle^{k / 2-1} \mathscr{L}_{p}\left(k / 2, \mathbf{f}_{\infty} / K, \psi_{K}\right)^{2}}{u_{K}^{2} \alpha(\mathfrak{p}, k)^{2} L_{p}\left(k / 2, k, \mathbf{f}_{\infty}, \psi_{1}\right) L_{p}\left(k / 2, k, \mathbf{f}_{\infty}, \psi_{2}\right)} .
$$

Remark 5.2. The function $\eta_{w}(k)$ does not depend on the choice of the characters $\psi_{1}, \psi_{2}$ made in the proof, because by (5.7), the values of $\eta_{w}(k)$ is independent of these choices for a set of values of $k$ that accumulates to 2 . The same argument also shows that $\eta_{w}=\eta_{-w}$. 
Proposition 5.3. Let $\psi_{1}, \psi_{2}$ be a pair of quadratic Hecke characters of $F$, which are unramified at the primes dividing $\mathfrak{n}$, and which correspond to a CM extension $K / F$ and a genus character $\psi_{K}$ of $K$. In the case where $\mathfrak{n}^{+} \mathfrak{n}^{-}$is the square of an ideal, assume the condition

$$
\alpha(\mathfrak{p}, E / F)=-\operatorname{sgn}\left(\psi_{1}\right) \cdot \epsilon_{E / F} .
$$

Then for $k \in U$,

$$
\begin{aligned}
& \mathscr{L}_{p}\left(k / 2, \mathbf{f}_{\infty} / K, \psi_{K}\right)^{2} \\
& \quad=u_{K}^{2}\left\langle D_{K}\right\rangle^{1-k / 2} \alpha(\mathfrak{p}, k)^{2} \eta_{\operatorname{sig}\left(\psi_{1}\right)}(k) L_{p}\left(k / 2, k, \mathbf{f}_{\infty}, \psi_{1}\right) L_{p}\left(k / 2, k, \mathbf{f}_{\infty}, \psi_{2}\right) .
\end{aligned}
$$

Proof. The computations in the proof of Proposition 5.1, show that equation (5.13) holds for $k \in U \cap \mathbb{Z}^{\geq 2}$, with $k \equiv 2 \bmod 2(p-1)$ (if $\mathfrak{p}$ is inert in $K$, i.e., if $\psi_{1}(\mathfrak{p})=$ $\psi_{2}(\mathfrak{p})$, then we use Theorem 3.13 instead of Theorem 3.14 in the computation). Since both sides of (5.13) are analytic functions of $k$, they must be equal.

We now come to the main theorem. We refer to the notations of Section 4.5.

Theorem 5.4. Let $\psi_{1}$ be a quadratic Hecke character of $F$, of conductor prime to $\mathfrak{n}=\mathfrak{p} \mathfrak{n}^{+} \mathfrak{n}^{-}$. Assume the following:

$$
\begin{gathered}
\psi_{1}(\mathfrak{p})=\alpha(\mathfrak{p}, E / F), \\
\epsilon_{E / F}\left(\psi_{1}\right)=-1 .
\end{gathered}
$$

Then

(1) The function $L_{p}\left(k / 2, k, \mathbf{f}_{\infty}, \psi_{1}\right)$ vanishes to order at least two at $k=2$.

(2) There exists $\mathbf{P}_{\psi_{1}} \in\left(E\left(F^{\psi_{1}}\right) \otimes \mathbb{Q}\right)_{\psi_{1}}$, and $l \in \mathbb{Q}^{\times}$, such that

$$
\left.\frac{d^{2}}{d k^{2}} L_{p}\left(k / 2, k, \mathbf{f}_{\infty}, \psi_{1}\right)\right|_{k=2}=l\left(\log N o r m_{E / F_{\mathfrak{p}}}\left(\mathbf{P}_{\psi_{1}}\right)\right)^{2} .
$$

(3) The element $\mathbf{P}_{\psi_{1}}$ is of infinite order if and only if $L^{\prime}\left(1, E / F, \psi_{1}\right) \neq 0$.

Proof. The proof of assertion (1) in the case when $\psi_{1}$ is trivial and $\alpha(\mathfrak{p}, E / F)=1$, is already given in the introduction. The proof for general $\psi_{1}$ with $\psi_{1}(\mathfrak{p})=\alpha(\mathfrak{p}, E / F)$ is similar. Notice that assertion (1) does not require the conductor $\mathfrak{n}$ to be of the form $\mathfrak{p}^{+} \mathfrak{n}^{-}$.

To prove the remaining claims, consider the set of quadratic Hecke characters $\psi_{2}$ of $F$, of conductors relatively prime to $\mathfrak{n}$ and $c_{\psi_{1}}$, that satisfies the following conditions:

(1) $\operatorname{sig}\left(\psi_{2}\right)=-\operatorname{sig}\left(\psi_{1}\right)$. 
(2) $\psi_{2}(\mathfrak{l})=\psi_{1}(\mathfrak{l})$ for $\mathfrak{l} \mid \mathfrak{n}^{+}$.

(3) $\psi_{2}(\mathfrak{l})=-\psi_{1}(\mathfrak{l})$ for $\mathfrak{l} \mid \mathfrak{p} \mathfrak{n}^{-}$.

Again by (5.5), one has $\epsilon_{E / F}\left(\psi_{2}\right)=-\epsilon_{E / F}\left(\psi_{1}\right)=1$ for any such $\psi_{2}$. So by [15], we can pick a $\psi_{2}$ such that $L\left(1, E / F, \psi_{2}\right) \neq 0$. Since $\psi_{2}(\mathfrak{p})=-\psi_{1}(\mathfrak{p})=$ $-\alpha(\mathfrak{p}, E / F), L_{p}\left(s, E / F, \psi_{2}\right)$ does not have an exceptional zero at $s=1$, so we have $L_{p}\left(1, E / F, \psi_{2}\right) \in \mathbb{Q}^{\times}$.

Thus we are in the setting of Proposition 5.3 (notice that, in the case where $\mathfrak{n}^{+} \mathfrak{n}^{-}$ is a square, conditions (5.14) imply (5.12)). Hence (5.13) holds. Note that since $\psi_{1} \psi_{2}(\mathfrak{p})=-1$, the prime $\mathfrak{p}$ is inert in $K$.

In our case $L_{p}\left(k / 2, k, \mathbf{f}_{\infty}, \psi_{1}\right)$ vanishes to order two at $k=2$, while the other functions that occur on the right hand side of (5.13) does not vanish. It follows, on taking second derivative at $k=2$, that

$$
\begin{gathered}
\left.u_{K}^{2} \eta_{\operatorname{sig}\left(\psi_{1}\right)}(2) L_{p}\left(1, E / F, \psi_{2}\right) \frac{d^{2}}{d k^{2}} L_{p}\left(k / 2, k, \mathbf{f}_{\infty}, \psi_{1}\right)\right|_{k=2} \\
=\left.\frac{d^{2}}{d k^{2}} \mathscr{L}\left(k, \mathbf{f}_{\infty} / K, \psi_{K}\right)^{2}\right|_{k=2} \\
=2\left[\left.\frac{d}{d k} \mathscr{L}\left(k, \mathbf{f}_{\infty} / K, \psi_{K}\right)\right|_{k=2}\right]^{2} .
\end{gathered}
$$

By Proposition 4.5 (noting conditions (5.14)),

$$
\left.\frac{d}{d k} \mathscr{L}\left(k, \mathbf{f}_{\infty} / K, \psi_{K}\right)\right|_{k=2}=\operatorname{logNorm}_{E / F_{\mathfrak{p}}}\left(\mathbf{P}_{\psi_{1}}\right)
$$

with $\mathbf{P}_{\psi_{1}} \in\left(E\left(F^{\psi_{1}}\right) \otimes \mathbb{Q}\right)_{\psi_{1}}$, and is non-torsion if and only if $L^{\prime}\left(1, E / F, \psi_{1}\right) \neq 0$. Hence we obtain the proof, by setting

$$
\begin{aligned}
l^{-1} & =\frac{1}{2} u_{K}^{2} \eta_{\operatorname{sig}\left(\psi_{1}\right)}(2) L_{p}\left(1, E / F, \psi_{2}\right) \\
& =u_{K}^{2} \eta_{\operatorname{sig}\left(\psi_{1}\right)}(2) L^{\operatorname{alg}}\left(1, E / F, \psi_{2}\right) .
\end{aligned}
$$

It remains to see that $l \in \mathbb{Q}^{\times}$. Note that, by Proposition 5.1, the expression

$$
i^{d} \frac{\Omega_{\mathbf{f}_{E}}^{\operatorname{sig}\left(\psi_{1}\right)} \Omega_{\mathbf{f}_{E}}^{-\operatorname{sig}\left(\psi_{1}\right)}}{\left\langle\mathbf{f}_{E}, \mathbf{f}_{E}\right\rangle}
$$

lies in $\mathbb{Q}^{\times}$, while the expression $\left\langle\Phi_{E}, \Phi_{E}\right\rangle$ lies in $\mathbb{Q}^{\times}$, due to our normalization condition that $\Phi_{E}$ is integer-valued. Hence $\eta_{\operatorname{sig}\left(\psi_{1}\right)}(2) \in \mathbb{Q}^{\times}$, which gives the result since $L^{\mathrm{alg}}\left(1, E / F, \psi_{2}\right) \in \mathbb{Q}^{\times}$.

Note that when $[F: \mathbb{Q}]$ is even, then the conductor $\mathfrak{n}$ of $E / F$ can always be written in the form $\mathfrak{p} \mathfrak{n}^{+} \mathfrak{n}^{-}$, for instance by taking $\mathfrak{n}^{-}=\mathcal{O}_{F}$. If $[F: \mathbb{Q}]$ is odd, 
then this condition is equivalent to requiring that $E / F$ has multiplicative reduction at some place other than $\mathfrak{p}$. In the next section, we are going to establish Theorem 5.4 without this condition, at least when $F=\mathbb{Q}$.

Remark 5.5. We can be more precise about the value of $l \bmod \left(\mathbb{Q}^{\times}\right)^{2}$. Let $\delta$ be a quadratic Hecke character of $F$, with conductor relatively prime to that of $\psi_{1}$ and $\psi_{2}$, and satisfying the following conditions (whose existence follows again from [15]):

(1) $\operatorname{sig}(\delta)=\operatorname{sig}\left(\psi_{1}\right)$.

(2) $\delta(\mathfrak{l})=\psi_{1}(\mathfrak{l})$ for $\mathfrak{l} \mid \mathfrak{n}^{+} \mathfrak{n}^{-}$.

(3) $\delta(\mathfrak{p})=-\psi_{1}(\mathfrak{p})$.

(4) $L(1, E / F, \delta) \neq 0$.

Let $\tilde{K} / F$ be the CM extension cuts out by the character $\tilde{\zeta}:=\delta \psi_{2}$, and $\psi_{\widetilde{K}}$ the genus character of $\widetilde{K}$ corresponding to the pair by $\delta, \chi_{2}$. Then as a consequence of equation (3.10), we have

$$
\left\langle\Phi_{E}, \Phi_{E}\right\rangle^{-1}=L^{\mathrm{alg}}\left(1, \mathbf{f}_{E} / \tilde{K}, \psi_{\tilde{K}}\right) \bmod \left(\mathbb{Q}^{\times}\right)^{2} .
$$

On the other hand, the same calculations that lead to (5.11) gives (noting that $\operatorname{sig}(\delta)=$ $\left.\operatorname{sig}\left(\psi_{1}\right)\right)$

$$
\begin{aligned}
L^{\mathrm{alg}}\left(1, \mathbf{f}_{E} / \widetilde{K}, \psi_{\tilde{K}}\right)=\langle & \left.\Phi_{E}, \Phi_{E}\right\rangle^{-1} \cdot \eta_{\operatorname{sig}\left(\psi_{1}\right)}(2) \\
& \cdot L^{\operatorname{alg}}(1, E / F, \delta) \cdot L^{\operatorname{alg}}\left(1, E / F, \psi_{2}\right) \bmod \left(\mathbb{Q}^{\times}\right)^{2} .
\end{aligned}
$$

Combining (5.15), (5.16) and (5.17), we thus obtain

$$
l=L^{\mathrm{alg}}(1, E / F, \delta) \bmod \left(\mathbb{Q}^{\times}\right)^{2} .
$$

In the case $F=\mathbb{Q}$, this gives part (4) of Theorem 5.4 of [4].

Suppose that we take $\psi_{1}$ to be trivial in Theorem 5.4; in particular, we have $\alpha(\mathfrak{p}, E / F)=1$, i.e., $E$ is split multiplicative at $\mathfrak{p}$. Then we can give another formulation of the statement of the theorem. Thus let $Q_{E} \in F_{\mathfrak{p}}^{\times}$be the Tate period, $q_{E}=\mathcal{N}_{F_{\mathfrak{p}} / \mathbb{Q}_{p}} Q_{E}$, and $\Theta_{E / F_{\mathfrak{p}}}$ be the Tate uniformization for $E / F_{\mathfrak{p}}$ :

$$
\Theta_{E / F_{\mathfrak{p}}}: F_{\mathfrak{p}}^{\times} / Q_{E}^{\mathbb{Z}} \stackrel{\sim}{\longrightarrow} E\left(F_{\mathfrak{p}}\right) .
$$

For any $\sigma \in \operatorname{Hom}(F, \overline{\mathbb{Q}})=\operatorname{Hom}\left(F, \overline{\mathbb{Q}}_{p}\right)=\operatorname{Hom}\left(F_{\mathfrak{p}}, \overline{\mathbb{Q}}_{p}\right)$, let $F^{\sigma}$ be the totally real field obtained by conjugating $F$ with $\sigma$, with $\mathfrak{p}^{\sigma}$ the prime of $F^{\sigma}$ above $p$. Similarly, let $E^{\sigma} / F^{\sigma}$ be the elliptic curve obtained by conjugating $E / F$ with $\sigma$. Then $E^{\sigma}$ is split multiplicative at $\mathfrak{p}^{\sigma}$, with Tate period $Q_{E}^{\sigma} \in\left(F_{\mathfrak{p}^{\sigma}}^{\sigma}\right)^{\times}$. We choose the Tate 
uniformization $\Theta_{E^{\sigma} / F_{p^{\sigma}}}$ for $E^{\sigma} / F_{p^{\sigma}}^{\sigma}$, so that the following diagram commutes (the vertical arrows being the natural maps obtained by conjugating with $\sigma$ ):

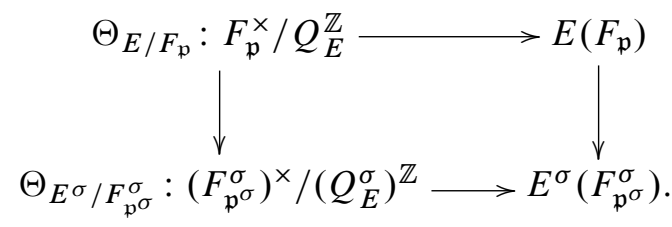

Define $\log _{E} \sigma / F_{p^{\sigma}}^{\sigma}$ for each $\sigma \in \operatorname{Hom}\left(F, \overline{\mathbb{Q}}_{p}\right)$ using these compatible families of Tate uniformizations. Then given any $\mathbf{P} \in E(F) \subset E\left(F_{\mathfrak{p}}\right)$, write

$$
\mathbf{P}=\Theta_{E / F_{\mathfrak{p}}}(z) \text { with } z \in F_{\mathfrak{p}}^{\times} .
$$

Then

$$
\begin{aligned}
\log _{\operatorname{Norm}_{E / F_{\mathfrak{p}}} \mathbf{P}}=\log _{q_{E}} \mathcal{N}_{F_{\mathfrak{p}} / \mathbb{Q}_{p}} z \\
=\sum_{\sigma \in \operatorname{Hom}\left(F, \overline{\mathbb{Q}}_{p}\right)} \log _{Q_{E}^{\sigma} z^{\sigma} \quad \text { by Proposition } 2.29} \log _{E^{\sigma} / F_{p^{\sigma}}^{\sigma}} \Theta_{E^{\sigma} / F_{\mathfrak{p}}^{\sigma} \sigma}\left(z^{\sigma}\right) \\
=\sum_{\sigma \in \operatorname{Hom}\left(F, \overline{\mathbb{Q}}_{p}\right)} \sum_{\sigma \in \operatorname{Hom}\left(F, \overline{\mathbb{Q}}_{p}\right)} \log _{E^{\sigma} / F_{p^{\sigma}}^{\sigma}}\left(\Theta_{E / F_{\mathfrak{p}}}(z)\right)^{\sigma} \\
=\sum_{\sigma \in \operatorname{Hom}\left(F, \overline{\mathbb{Q}}_{p}\right)} \log _{E^{\sigma} / F_{p^{\sigma}}^{\sigma}} \mathbf{P}^{\sigma}
\end{aligned}
$$

with $\mathbf{P}^{\sigma} \in E^{\sigma}\left(F^{\sigma}\right)$.

Thus we obtain

Corollary 5.6. If in addition to the hypothesis of Theorem 5.4, we have E/F being split multiplicative at $\mathfrak{p}$, then

$$
\left.\frac{d^{2}}{d k^{2}} L_{p}\left(k / 2, k, \mathbf{f}_{\infty}\right)\right|_{k=2}=l\left(\sum_{\sigma \in \operatorname{Hom}\left(F, \overline{\mathbb{Q}}_{p}\right)} \log _{E^{\sigma} / F_{p^{\sigma}}^{\sigma}} \mathbf{P}^{\sigma}\right)^{2}
$$

for $\mathbf{P} \in E(F)$, and $l \in \mathbb{Q}^{\times}$. The point $\mathbf{P}$ is non-torsion, if and only if $L^{\prime}(1, E / F) \neq 0$.

\section{Base change arguments}

In this final section, let $E / \mathbb{Q}$ be an elliptic curve, which is modular by [37], [32], [8], corresponding to a weight two elliptic eigenform $f_{E}$ (i.e., a Hilbert eigenform 
over $\mathbb{Q}$ ). Let $N$ be the conductor of $E / \mathbb{Q}$ (and hence that of $f_{E}$ ), and as in the previous section $E / \mathbb{Q}$ is multiplicative at $p$. We would like to establish Theorem 5.4 without assuming that $E / \mathbb{Q}$ is multiplicative at some prime other than $p$.

The idea is to consider the base change $E / F$, where $F$ is a suitably chosen real quadratic extension of $\mathbb{Q}$. Theorem 5.4 is known for $E / F$. Suitable descent arguments allow us to get the statement for $E / \mathbb{Q}$.

First, we would like to establish a factorization formula, relating the $p$-adic $L$ functions of a elliptic modular form, and the $p$-adic $L$-function attached to the corresponding Hilbert modular form obtained by base change. The situation is parallel to Proposition 5.1.

The reader may notice that most of the arguments in this section is not specific to the ground field $\mathbb{Q}$. Indeed, the only place where this is essential is the proof of Lemma 6.1 and Proposition 6.4 below, where we make use of a non-vanishing result of Rohrlich [28], [29] on twisted $L$-values, which was proved only for the ground field $\mathbb{Q}$. If the result of Rohrlich can be extended to general totally real fields, then everything in this section would work without the restriction to $\mathbb{Q}$.

Here is the setup: let $\psi_{1}, \psi_{2}$ be two quadratic Hecke characters of $\mathbb{Q}$ (i.e., Dirichlet characters), whose conductors $c_{\psi_{1}}, c_{\psi_{2}}$ are relatively prime to each other, and also prime to the integer $N$ above. Assume that $\operatorname{sig}\left(\psi_{1}\right)=\operatorname{sig}\left(\psi_{2}\right)=w \in\{ \pm 1\}$. Then as in Section 3.1, the character $\psi_{1} \psi_{2}$ defines a quadratic extension $F$ of $\mathbb{Q}$, which is real quadratic by the condition on the signatures. The pair $\psi, \psi_{2}$ corresponds to a genus character $\psi_{F}$ of $F$. One sees that $\operatorname{sig}\left(\psi_{F}\right)=w \cdot \underline{1}=(w, w)$. In conforming with the assumption in this paper, $p$ will be inert in $F$, so this amounts to requiring that $\psi_{1}(p)=-\psi_{2}(p)$.

First recall the formalism of the base change operation. Let $g$ be an elliptic cuspidal eigenform of weight $k \geq 2$, of level $N$. To simplify the arguments, assume that $k$ is even, and that the character of $g$ is trivial. Given the real quadratic extension $F / \mathbb{Q}$, denote by $\mathbf{g}$ the base change of $g$ from $\mathbb{Q}$ to $F$, which is a cuspidal Hilbert eigenform of parallel weight $k$, known as the Doi-Naganuma lift of $g$ (cf. [9], Section 1.7). For simplicity we assume that the level $N$ is relatively prime to the discriminant of $F$.

By the definition of base change we have the following relation between the $L$ function of $\mathbf{g}$ and that of $g$. Denoting by $\xi$ the Dirichlet character corresponding to the real quadratic extension $F / \mathbb{Q}$, we have

$$
L(s, \mathbf{g})=L(s, g) \cdot L(s, g, \xi) .
$$

This is equivalent to the following relations between the (normalized) Fourier coefficients of $g$ and $\mathbf{g}$ (cf. [9], p. 89). For any (rational) prime $v$ let $c(v, g)$ be the $v$-th Fourier coefficient of $g$. First suppose that $v \nmid N$. Let $\alpha(v, g), \beta(v, g)$ be the roots of the characteristic polynomial

$$
X^{2}-c(v, g) X+v^{k-1} .
$$


Similarly if $\mathfrak{v}$ is a prime of $F$, with $\mathfrak{v} \nmid N$, let $\alpha(\mathfrak{v}, \mathbf{g}), \beta(\mathfrak{v}, \mathbf{g})$ be the roots of

$$
X^{2}-C(\mathfrak{v}, \mathbf{g}) X+\mathcal{N} \mathfrak{v}^{k-1} .
$$

If $v$ splits in $F$, i.e., if $v \mathcal{O}_{F}=\mathfrak{v} \mathfrak{v}^{\prime}$, then we have

$$
\begin{gathered}
\alpha(\mathfrak{v}, \mathbf{g})=\alpha\left(\mathfrak{v}^{\prime}, \mathbf{g}\right)=\alpha(v, g) \\
\beta(\mathfrak{v}, \mathbf{g})=\beta\left(\mathfrak{v}^{\prime}, \mathbf{g}\right)=\beta(v, g) \\
C(\mathfrak{v}, \mathbf{g})=C\left(\mathfrak{v}^{\prime}, \mathbf{g}\right)=c(v, g) .
\end{gathered}
$$

On the other hand, if $v$ is inert in $F$, so that $\mathfrak{v}:=v \mathcal{O}_{F}$ is a prime, then

$$
\begin{gathered}
\alpha(\mathfrak{v}, \mathbf{g})=\alpha(v, g)^{2}, \quad \beta(\mathfrak{v}, \mathbf{g})=\beta(v, g)^{2} \\
C(\mathfrak{v}, \mathbf{g})=\alpha(v)^{2}+\beta(v)^{2}=c(v, g)^{2}-2 v^{k-1} .
\end{gathered}
$$

One has similar relations for the Fourier coefficients for primes dividing $D_{F}$ or the level $N$ of $g$. Namely if $v \mid N$, then $C(\mathfrak{v}, \mathbf{g})=C\left(\mathfrak{v}^{\prime}, \mathbf{g}\right)=c(v, g)$ when $v \mathcal{O}_{F}=\mathfrak{v} \mathfrak{v}^{\prime}$ splits, and $C(\mathfrak{v}, \mathbf{g})=c(v, g)^{2}$ when $v \mathcal{O}_{F}=\mathfrak{v}$ is inert. Finally if $v$ ramifies in $F$, then $C(\mathfrak{v}, \mathbf{g})=c(v, g)$ for the prime $\mathfrak{v}$ of $F$ above $v$.

From these relations, one see in particular that $\mathbb{Q}(\mathbf{g}) \subset \mathbb{Q}(g)$.

Lemma 6.1. With the above notations, the expression

$$
\kappa(g):=D_{F}^{-1 / 2}\left(\Omega_{g}^{w}\right)^{2}\left(\Omega_{\mathrm{g}}^{w}\right)^{-1}
$$

lies in $\mathbb{Q}(g)$.

Proof. Let $T$ be the set of finite order Hecke characters $\chi$ of $\mathbb{Q}$, that are unramified at primes outside $p$ (including the infinite place); in classical language, this is the set of even Dirichlet characters with conductors a power of $p$. Consider the following condition:

$$
L\left(k-1, g, \psi_{1} \chi\right) \neq 0, L\left(k-1, g, \psi_{2} \chi\right) \neq 0 .
$$

If $k \geq 4$, then by Proposition 2 of [30], condition (6.4) is satisfied for any $\chi \in T$. For $k=2$, then by the main result of [28], [29], (6.4) is satisfied for all but finitely many $\chi \in T$. Thus pick a non-trivial $\tilde{\chi} \in T$ that satisfies (6.4) (so in particular $p$ divides the conductor of $\tilde{\chi}$ ).

Let $\tilde{\chi}_{F}$ be the Hecke character of $F$ obtained by pulling back $\tilde{\chi}$ via the norm map $\mathcal{N}_{F / \mathbb{Q}}$, i.e.,

$$
\tilde{\chi}_{F}=\tilde{\chi} \circ \mathcal{N}_{F / \mathbb{Q}} .
$$

Then we have the following identity of the complex $L$-functions:

$$
L\left(s, \mathbf{g}, \psi_{F} \tilde{\chi}_{F}\right)=L\left(s, g, \psi_{1} \tilde{\chi}\right) \cdot L\left(s, g, \psi_{2} \tilde{\chi}\right) .
$$


So in particular

$$
L\left(k-1, \mathbf{g}, \psi_{F} \tilde{\chi}_{F}\right)=L\left(k-1, g, \psi_{1} \tilde{\chi}\right) \cdot L\left(k-1, g, \psi_{2} \tilde{\chi}\right) \neq 0 .
$$

Again from Proposition 7.1 of Appendix I, we have the following identities relating the conductors and the Gauss sums:

$$
\begin{gathered}
\mathcal{N}_{F / \mathbb{Q}} c_{\psi_{F} \tilde{\chi}_{F}}=\mathcal{N}_{F / \mathbb{Q}} c_{\tilde{\chi}_{F}}=c_{\tilde{\chi}}^{2}, \\
D_{F}=c_{\psi_{1}} c_{\psi_{2}}, \\
\tau\left(\left(\psi_{F} \tilde{\chi}_{F}\right)^{-1}\right)=D_{F}^{-1 / 2} \tau\left(\left(\psi_{1} \tilde{\chi}\right)^{-1}\right) \cdot \tau\left(\left(\psi_{2} \tilde{\chi}\right)^{-1}\right) .
\end{gathered}
$$

Making use of equations (5.1) (applied to $L\left(k-1, \mathbf{g}, \psi_{F} \tilde{\chi}_{F}\right), L\left(k-1, g, \psi_{1} \tilde{\chi}\right)$ and $\left.L\left(k-1, g, \psi_{2} \tilde{\chi}\right)\right),(6.5)$ and (6.6), we obtain by direct computation

$$
\kappa(g)=\frac{L^{\operatorname{alg}}\left(k-1, \mathbf{g}, \psi_{F} \tilde{\chi}_{F}\right)}{L^{\operatorname{alg}}\left(k-1, g, \psi_{1} \tilde{\chi}\right) \cdot L^{\operatorname{alg}}\left(k-1, g, \psi_{2} \tilde{\chi}\right)}
$$

with $\kappa(g)$ defined as in (6.3).

Since the numbers

$$
L^{\operatorname{alg}}\left(k-1, \mathbf{g}, \psi_{F} \tilde{\chi}_{F}\right), \quad L^{\operatorname{alg}}\left(k-1, g, \psi_{1} \tilde{\chi}\right) \quad \text { and } \quad L^{\operatorname{alg}}\left(k-1, g, \psi_{2} \tilde{\chi}\right)
$$

are algebraic numbers, we deduce that $\kappa(g)$ is algebraic. To pin down its field of definition more precisely, let $h \in \operatorname{Aut}(\overline{\mathbb{Q}} / \mathbb{Q}(g))$ be arbitrary. Since $\mathbb{Q}(\mathbf{g}) \subset \mathbb{Q}(g)$, we also have $h \in \operatorname{Aut}(\overline{\mathbb{Q}} / \mathbb{Q}(\mathbf{g}))$.

Noticing that since $\psi_{1}, \psi_{2}$ and $\psi_{F}$ take values in $\{ \pm 1\}$, we see, by (5.2),

$$
\begin{aligned}
L^{\mathrm{alg}}\left(k-1, g, \psi_{1} \tilde{\chi}^{h}\right) & =L^{\mathrm{alg}}\left(k-1, g, \psi_{1} \tilde{\chi}\right)^{h} \neq 0, \\
L^{\mathrm{alg}}\left(k-1, g, \psi_{2} \tilde{\chi}^{h}\right) & =L^{\mathrm{alg}}\left(k-1, g, \psi_{2} \tilde{\chi}\right)^{h} \neq 0, \\
L^{\mathrm{alg}}\left(k-1, \mathbf{g}, \psi_{F} \tilde{\chi}_{F}^{h}\right) & =L^{\mathrm{alg}}\left(k-1, \mathbf{g}, \psi_{F} \tilde{\chi}_{F}\right)^{h} \neq 0 .
\end{aligned}
$$

In particular, in the above computation, we could replace $\tilde{\chi}$ by $\tilde{\chi}^{h}$, and so equation (6.7) holds with $\tilde{\chi}$ replaced by $\tilde{\chi}^{h}$. We deduce

$$
\begin{aligned}
\kappa(g) & =\frac{L^{\operatorname{alg}}\left(k-1, \mathbf{g}, \psi_{F} \tilde{\chi}_{F}\right)}{L^{\operatorname{alg}}\left(k-1, g, \psi_{1} \tilde{\chi}\right) \cdot L^{\operatorname{alg}}\left(k-1, g, \psi_{2} \tilde{\chi}\right)} \\
& =\frac{L^{\operatorname{alg}}\left(k-1, \mathbf{g}, \psi_{F} \tilde{\chi}_{F}^{h}\right)}{L^{\operatorname{alg}}\left(k-1, g, \psi_{1} \tilde{\chi}^{h}\right) \cdot L^{\operatorname{alg}}\left(k-1, g, \psi_{2} \tilde{\chi}^{h}\right)} \\
& =\left(\frac{L^{\operatorname{alg}}\left(k-1, \mathbf{g}, \psi_{F} \tilde{\chi}_{F}\right)}{L^{\operatorname{alg}}\left(k-1, g, \psi_{1} \tilde{\chi}\right) \cdot L^{\operatorname{alg}}\left(k-1, g, \psi_{2} \tilde{\chi}\right)}\right)^{h} \\
& =\kappa(g)^{h}
\end{aligned}
$$

which implies that $\kappa(g) \in \mathbb{Q}(g)$, as required. 
Now assume that $g$ is ordinary at $p$. For the sake of simplifying the arguments, assume that the weight $k$ satisfies $k \equiv 2 \bmod p-1$. As in the proof of Lemma 6.1, for any finite order Hecke character $\chi$ of $\mathbb{Q}$, let $\chi_{F}:=\chi \circ \mathcal{N}_{F / \mathbb{Q}}$ be the corresponding Hecke character of $F$.

Lemma 6.2. For any finite order Hecke character $\chi$ of $\mathbb{Q}$, unramified at places outside $p$, we have

$$
L_{p}\left(k-1, \mathbf{g}, \psi_{F} \chi_{F}\right)=\kappa(g) L_{p}\left(k-1, g, \psi_{1} \chi\right) \cdot L_{p}\left(k-1, g, \psi_{2} \chi\right) .
$$

Proof. Similar to the computations leading to equation (6.7) in the proof of Lemma 6.1, we have

$$
L^{\mathrm{alg}}\left(k-1, \mathbf{g}, \psi_{F} \chi_{F}\right)=\kappa(g) L^{\mathrm{alg}}\left(k-1, g, \psi_{1} \chi\right) \cdot L^{\mathrm{alg}}\left(k-1, g, \psi_{2} \chi\right) .
$$

Lemma 6.2 then follows by combining equation (6.8) with equation (5.3) (applied to $L_{p}\left(k-1, \mathbf{g}, \psi_{F} \chi_{F}\right), L_{p}\left(k-1, g, \psi_{1} \chi\right)$ and $\left.L_{p}\left(k-1, g, \psi_{2} \chi\right)\right)$, and the computation (using $\psi_{1}(p)=-\psi_{2}(p)$ and that $\alpha(\mathfrak{p}, \mathbf{g})=\alpha(p, g)^{2}$ ):

$$
\begin{aligned}
\left(1-\frac{\psi_{1} \chi(p) p^{k-2}}{\alpha(p, g)}\right)\left(1-\frac{\psi_{2} \chi(p) p^{k-2}}{\alpha(p, g)}\right) & =\left(1-\frac{\left(\chi(p) p^{k-2}\right)^{2}}{\alpha(p, g)^{2}}\right) \\
& =\left(1-\frac{\psi_{F} \chi_{F}(\mathfrak{p}) \mathcal{N} \mathfrak{p}^{k-2}}{\alpha(\mathfrak{p}, \mathbf{g})}\right)
\end{aligned}
$$

(here we have used $\psi_{F}(\mathfrak{p})=1$, because the image of $\mathfrak{p}=p \mathcal{O}_{F}$ in $\operatorname{Pic}\left(\mathcal{O}_{F}\right)$ comes from the image of $p$ in $\operatorname{Pic}(\mathbb{Z})$, which is trivial). Similarly,

$$
\begin{aligned}
(1- & \left.\frac{\left(\psi_{1} \chi\right)^{-1}(p) \beta(p, g)}{p^{k-1}}\right)\left(1-\frac{\left(\psi_{2} \chi\right)^{-1}(p) \beta(p, g)}{p^{k-1}}\right) \\
& =\left(1-\frac{\left(\psi_{F} \chi_{F}\right)^{-1}(\mathfrak{p}) \beta(\mathfrak{p}, \mathbf{g})}{\mathcal{N} \mathfrak{p}^{k-1}}\right) .
\end{aligned}
$$

Proposition 6.3. We have the factorization formula of p-adic L-function:

$$
L_{p}\left(s, \mathbf{g}, \psi_{F}\right)=\kappa(g) L_{p}\left(s, g, \psi_{1}\right) \cdot L_{p}\left(s, g, \psi_{2}\right) \text {. }
$$

Proof. The proof follows the same lines of argument of Proposition 9.3 of [26], using Lemma 6.2 above.

We now come back to the setting of the beginning of this section. Let $\mathbf{f}_{E}$ be the base change of $f_{E}$ to $F$. Then $\mathbf{f}_{E}$ corresponds to $E / F$ (so $E / F$ is modular). Let $f_{\infty}$ be the Hida family lifting $f_{E}$. The Hida family $\mathbf{f}_{\infty}$ lifting $\mathbf{f}_{E}$ can be obtained from $f_{\infty}$ by base change. More precisely we can construct the $\Lambda^{\dagger}$-adic eigenform 
$\mathbf{f}_{\infty}$ using formula similar to (6.1) and (6.2), i.e., for primes $\mathfrak{v}$ of $F$ define $C\left(\mathfrak{v}, \mathbf{f}_{\infty}\right)$ by the following formula:

$$
C\left(\mathfrak{v}, \mathbf{f}_{\infty}\right)=C\left(\mathfrak{v}^{\prime}, \mathbf{f}_{\infty}\right)=c\left(v, f_{\infty}\right) \text { if } v \mathcal{O}_{F}=\mathfrak{v} \mathfrak{v}^{\prime} \text { splits. }
$$

If $v \mathcal{O}_{F}=\mathfrak{v}$ is inert in $F$, then

$$
C\left(\mathfrak{v}, \mathbf{f}_{\infty}\right)= \begin{cases}c\left(v, f_{\infty}\right)^{2}-2 v\langle v\rangle^{k-2} & \text { if } v \nmid N \text { (here } k \text { is a variable), } \\ c\left(v, f_{\infty}\right)^{2} & \text { if } v \mid N \text { (in particular for } v=p) .\end{cases}
$$

If $v$ ramifies in $F$, with $\mathfrak{v}$ the prime of $F$ above $v$, then

$$
C\left(\mathfrak{v}, \mathbf{f}_{\infty}\right)=c\left(v, f_{\infty}\right)
$$

One then defines $C\left(\mathfrak{m}, \mathbf{f}_{\infty}\right)$ for other ideals by (2.11). These data defines the $\Lambda^{\dagger}$-adic form $\mathbf{f}_{\infty}$, and for integers $k \in U \cap \mathbb{Z} \geq 2$ for a suitable $p$-adic neighbourhood $U$ of 2 , the weight $k$ specialization $\mathbf{f}_{k}$ of $\mathbf{f}_{\infty}$ is the cuspidal Hilbert eigenform that is the base change lift of the weight $k$ specialization $f_{k}$ of $f_{\infty}$. Let $f_{k}^{\#}$ and $\mathbf{f}_{k}^{\#}$ be the newforms attached to weight $k$ specializations of $f_{\infty}$ and $\mathbf{f}_{\infty}$ (so that $\mathbf{f}_{k}^{\#}$ is the base change lift of $f_{k}^{\#}$ to $F$ ).

Proposition 6.4. Up to shrinking $U$, there is a p-adic analytic function $\kappa(k)$, defined on $U$, such that for $k \in U \cap \mathbb{Z}^{\geq 2}$, with $k \equiv 2 \bmod p-1$, one has

$$
\kappa(k)=\kappa\left(f_{k}^{\#}\right) .
$$

Proof. With notations as in the proof of Lemma 6.1, choose a $\tilde{\chi} \in T$, whose conductor is divisible by $p$, such that

$$
L\left(1, E / F, \psi_{F} \tilde{\chi}_{F}\right), \quad L\left(1, E / \mathbb{Q}, \psi_{1} \tilde{\chi}\right) \quad \text { and } \quad L\left(1, E / \mathbb{Q}, \psi_{2} \tilde{\chi}\right)
$$

are non-zero. Since $p$ divides the conductor of $\tilde{\chi}$, we have by equation (5.3) that

$$
\begin{aligned}
L_{p}\left(1, E / \mathbb{Q}, \psi_{1} \tilde{\chi}\right) & =L^{\operatorname{alg}}\left(1, E / \mathbb{Q}, \psi_{1} \tilde{\chi}\right), \\
L_{p}\left(1, E / \mathbb{Q}, \psi_{2} \tilde{\chi}\right) & =L^{\operatorname{alg}}\left(1, E / \mathbb{Q}, \psi_{2} \tilde{\chi}\right), \\
L_{p}\left(1, E / F, \psi_{F} \tilde{\chi}_{F}\right) & =L^{\operatorname{alg}}\left(1, E / F, \psi_{F} \tilde{\chi}_{F}\right) .
\end{aligned}
$$

Hence up to shrinking $U$, we may assume that the $p$-adic analytic functions

$$
L_{p}\left(k-1, k, f_{\infty}, \psi_{1}\right), \quad L_{p}\left(k-1, k, f_{\infty}, \psi_{2}\right) \quad \text { and } \quad L_{p}\left(k-1, k, \mathbf{f}_{\infty}, \psi_{F}\right)
$$

are non-zero on $U$. Thus, if we define

$$
\kappa(k):=\frac{L_{p}\left(k-1, k, \mathbf{f}_{\infty}, \psi_{F}\right)}{L_{p}\left(k-1, k, f_{\infty}, \psi_{1}\right) L_{p}\left(k-1, k, f_{\infty}, \psi_{2}\right)},
$$


then $\kappa(k)$ is $p$-adic analytic on $U$, and Lemma 6.2 shows that, for $k \in U \cap \mathbb{Z}^{\geq 2}$, with $k \equiv 2 \bmod p-1$,

$$
\kappa(k)=\kappa\left(f_{k}^{\#}\right)
$$

as required.

We now draw the corollary that we need:

Corollary 6.5. For $k \in U$, we have

$$
L_{p}\left(k / 2, k, \mathbf{f}_{\infty}, \psi_{F}\right)=\kappa(k) L_{p}\left(k / 2, k, f_{\infty}, \psi_{1}\right) \cdot L_{p}\left(k / 2, k, f_{\infty}, \psi_{2}\right) .
$$

Proof. Propositions 6.3 and 6.4 show that this holds for integers $k \in U \cap \mathbb{Z} \geq 2$, with $k \equiv 2 \bmod p-1$. Since both sides are $p$-adic analytic functions of $k$, the result follows.

We are now in a position to extend Theorem 5.4 to the elliptic curve $E / \mathbb{Q}$, without assuming that $E / \mathbb{Q}$ is multiplicative at some prime other than $p$. Thus we are only assuming that $N=M p$, where $p$ does not divide $M$. Let $\psi_{1}$ be a quadratic Hecke character of $\mathbb{Q}$, with conductor relatively prime to $N$, satisfying

$$
\begin{gathered}
\psi_{1}(p)=\alpha(p):=\alpha(p, E / \mathbb{Q}), \\
\epsilon_{E / \mathbb{Q}}\left(\psi_{1}\right)=-1 .
\end{gathered}
$$

Let $\psi_{2}$ be a quadratic Hecke character of $\mathbb{Q}$, with conductor relatively prime to that of $\psi_{1}$ and to $N$, satisfying the following conditions:

(1) $\operatorname{sig}\left(\psi_{2}\right)=\operatorname{sig}\left(\psi_{1}\right)$.

(2) $\psi_{2}(p)=-\psi_{1}(p)$.

(3) $\psi_{2}(q)=\psi_{1}(q)$ for all primes $q$ dividing $M$.

(4) $L\left(1, E / \mathbb{Q}, \psi_{2}\right) \neq 0$.

Note that for any $\psi_{2}$ satisfying (1) to (3), we have (using (5.5) again)

$$
\epsilon_{E / \mathbb{Q}}\left(\psi_{2}\right)=-\epsilon_{E / \mathbb{Q}}\left(\psi_{1}\right)=1,
$$

so the existence of $\psi_{2}$ that satisfies (1) to (4) follows again from [15]. Note that (2) implies $\psi_{2}(p)=-\alpha(p, E / \mathbb{Q})$, so the $p$-adic $L$-function $L_{p}\left(s, E / \mathbb{Q}, \psi_{2}\right)$ does not have an exceptional zero at $s=1$. So $L_{p}\left(1, E / \mathbb{Q}, \psi_{2}\right) \in \mathbb{Q}^{\times}$.

Let $F$ be as above the real quadratic extension of $\mathbb{Q}$ cut out by $\psi_{1} \psi_{2}$, and $\psi_{F}$ the genus character of $F$ defined by the pair $\psi_{1}, \psi_{2}$.

Consider the base change $E / F$. We have

$$
\alpha(\mathfrak{p}, E / F)=\alpha(p, E / \mathbb{Q})^{2}=1=\psi_{F}(\mathfrak{p}) .
$$


On the other hand, the factorization formula for the complex $L$-functions,

$$
L\left(s, E / F, \psi_{F}\right)=L\left(s, E / \mathbb{Q}, \psi_{1}\right) \cdot L\left(s, E / \mathbb{Q}, \psi_{2}\right),
$$

together with the hypothesis that $L\left(1, E / \mathbb{Q}, \psi_{2}\right) \neq 0$, shows that the order of vanishing of $L\left(s, E / F, \psi_{F}\right)$ at $s=1$ coincides with that of $L\left(s, E / \mathbb{Q}, \psi_{1}\right)$, which is odd, i.e.,

$$
\epsilon_{E / F}\left(\psi_{F}\right)=\epsilon_{E / \mathbb{Q}}\left(\psi_{1}\right)=-1 .
$$

Since $[F: \mathbb{Q}]=2$ is even, we may apply Theorem 5.4 to the $p$-adic $L$-function $L_{p}\left(k / 2, k, \mathbf{f}_{\infty}, \psi_{F}\right)$ to get

$$
\left.\frac{d^{2}}{d k^{2}} L_{p}\left(k / 2, k, \mathbf{f}_{\infty}, \psi_{F}\right)\right|_{k=2}=l\left(\log \operatorname{Norm}_{E / F_{\mathfrak{p}}} \mathbf{P}_{\psi_{F}}\right)^{2},
$$

where $l \in \mathbb{Q}^{\times}$, and $\mathbf{P}_{\psi_{F}} \in\left(E\left(F^{\psi_{F}}\right) \otimes \mathbb{Q}\right)_{\psi_{F}}$ is non-torsion if and only if

$$
L^{\prime}\left(1, E / F, \psi_{F}\right) \neq 0 .
$$

By (6.10), and the fact that $L\left(1, E / \mathbb{Q}, \psi_{2}\right) \neq 0$, we have

$$
L^{\prime}\left(1, E / F, \psi_{F}\right)=L^{\prime}\left(1, E / \mathbb{Q}, \psi_{1}\right) \cdot L\left(1, E / \mathbb{Q}, \psi_{2}\right) .
$$

Hence

$$
L^{\prime}\left(1, E / F, \psi_{F}\right) \neq 0 \quad \text { if and only if } \quad L^{\prime}\left(1, E / \mathbb{Q}, \psi_{1}\right) \neq 0 .
$$

By (6.9), and the fact that both $L_{p}\left(k / 2, k, \mathbf{f}_{\infty}, \psi_{F}\right)$ and $L_{p}\left(k / 2, k, f_{\infty}, \psi_{1}\right)$ vanishes to order at least two at $k=2$, we have

$$
\left.\frac{d^{2}}{d k^{2}} L_{p}\left(k / 2, k, \mathbf{f}_{\infty}, \psi_{F}\right)\right|_{k=2}=\left.\kappa(2) L_{p}\left(1, E / \mathbb{Q}, \psi_{2}\right) \frac{d^{2}}{d k^{2}} L_{p}\left(k / 2, k, f_{\infty}, \psi_{1}\right)\right|_{k=2}
$$

with $\kappa(2) \in \mathbb{Q}^{\times}$by Lemma 6.1.

Define

$$
l^{\prime}:=\kappa(2)^{-1} \cdot L_{p}\left(1, E / \mathbb{Q}, \psi_{2}\right)^{-1} l=2^{-1} \kappa(2)^{-1} \cdot L^{\operatorname{alg}}\left(1, E / \mathbb{Q}, \psi_{2}\right)^{-1} l .
$$

Then $l^{\prime} \in \mathbb{Q}^{\times}$. It remains to deal with the term involving $\log \operatorname{Norm}_{E / F_{\mathfrak{p}}}$.

Notice that, in the notations of Section 3.1, we have

$$
F^{\psi_{F}}=\mathbb{Q}^{\psi_{1}, \psi_{2}}
$$

and thus $\mathbf{P}_{\psi_{F}} \in\left(E\left(\mathbb{Q}^{\psi_{1}, \psi_{2}}\right) \otimes \mathbb{Q}\right)_{\psi_{F}}$. As in Corollary 4.2, the assumption that $L\left(1, E / \mathbb{Q}, \psi_{2}\right) \neq 0$ implies, by the result of [24] (applied to $E / \mathbb{Q}$, so this case already follows from earlier work of Kolyvagin), that

$$
\mathbf{P}_{\psi_{F}} \in\left(E\left(\mathbb{Q}^{\psi_{1}}\right) \otimes \mathbb{Q}\right)_{\psi_{1}}
$$


Clearing out denominators, we may assume that $\mathbf{P}_{\psi_{F}} \in E\left(\mathbb{Q}^{\psi_{1}}\right)_{\psi_{1}}$ without affecting the value of $l \bmod \left(\mathbb{Q}^{\times}\right)^{2}$. In particular, in the notations of Section 4.5, the image of $\mathbf{P}_{\psi_{F}}$ in $E\left(\mathbb{Q}_{p^{2}}\right)$ lies in $E\left(\mathbb{Q}_{p^{2}}\right)_{\alpha(p)}$.

Let $q_{E} \in \mathbb{Q}_{p}^{\times}$be the Tate period of $E / \mathbb{Q}_{p}$, and let

$$
\Theta_{E / \mathbb{Q}_{p}}: \mathbb{C}_{p}^{\times} / q_{E}^{\mathbb{Z}} \stackrel{\sim}{\longrightarrow} E\left(\mathbb{C}_{p}\right)
$$

be the Tate uniformization for the $\mathbb{Q}_{p^{2}}$-points of $E / \mathbb{Q}_{p}$, which is also the Tate uniformization for $E / F_{\mathfrak{p}}$. Recall from Section 2.11, that the property of the Tate uniformization gives $E\left(\mathbb{Q}_{p^{2}}\right)_{\alpha_{p}}=\Theta_{E / \mathbb{Q}_{p}}\left(\mathbb{Q}_{p}\right)$, so in particular we have

$$
\mathbf{P}_{\psi_{F}}=\Theta_{E_{\mathbb{Q}_{p}}}(z)
$$

with $z \in \mathbb{Q}_{p}^{\times}$. So in particular

$$
\operatorname{logNorm}_{E / F_{\mathfrak{p}}}\left(\mathbf{P}_{\psi_{F}}\right)=\log _{q_{E}} \mathcal{N}_{F_{\mathfrak{p}} / \mathbb{Q}_{p}}(z)=2 \log _{q_{E}}(z)=2 \log _{E / \mathbb{Q}_{p}}\left(\mathbf{P}_{\psi_{F}}\right) .
$$

Thus to conclude, if we let

$$
l^{\prime \prime}:=2 \kappa(2)^{-1} L^{\mathrm{alg}}\left(1, E / \mathbb{Q}, \psi_{2}\right)^{-1} l,
$$

then

$$
\left.\frac{d^{2}}{d k^{2}} L_{p}\left(k / 2, k, f_{\infty}, \psi_{1}\right)\right|_{k=2}=l^{\prime \prime}\left(\log _{E / \mathbb{Q}_{p}} \mathbf{P}\right)^{2}
$$

with $\mathbf{P}=\mathbf{P}_{\psi_{F}} \in\left(E\left(\mathbb{Q}^{\psi_{1}}\right) \otimes \mathbb{Q}\right)_{\psi_{1}}$ non-torsion if and only if $L^{\prime}\left(1, E / \mathbb{Q}, \psi_{1}\right) \neq 0$.

Remark 6.6. In [5], equation (6.12) is applied to the study of the question of rationality of Stark-Heegner points. For this purpose, they need to know the value of $l^{\prime \prime}$ $\bmod \left(\mathbb{Q}^{\times}\right)^{2}$. See the discussion of Remark 5.5. These suggests that the conclusion of remark 5.5 holds for $E / \mathbb{Q}$ even without assuming that $E / \mathbb{Q}$ is multiplicative at some prime other than $p$. Thus for $\psi_{2}$ as above, we state the following conjectural formula:

$$
l^{\prime \prime} \stackrel{?}{=} L^{\mathrm{alg}}\left(1, E / \mathbb{Q}, \psi_{2}\right) \quad \bmod \left(\mathbb{Q}^{\times}\right)^{2} .
$$

On the other hand, using (6.11), (6.3) and (5.18), we have

$$
l^{\prime \prime}=L^{\operatorname{alg}}\left(1, E / \mathbb{Q}, \psi_{2}\right) \cdot 2 \frac{D_{F}^{1 / 2} \mathcal{N}_{F / \mathbb{Q}} \mathfrak{c}_{\delta} L(1, E / F, \delta)}{\tau(\delta)\left(\Omega_{f_{E}}^{\operatorname{sig}\left(\psi_{1}\right)}\right)^{2}} \bmod \left(\mathbb{Q}^{\times}\right)^{2},
$$

where $\delta$ is a quadratic Hecke character of $F$, unramified at the primes dividing the conductor of $E / F$, that satisfies 
(1) $\operatorname{sig}(\delta)=\operatorname{sig}\left(\psi_{F}\right)=\left(\operatorname{sig}\left(\psi_{1}\right), \operatorname{sig}\left(\psi_{1}\right)\right)$,

(2) $\delta(\mathfrak{p})=-\psi_{F}(\mathfrak{p})=-1$,

(3) $\delta(\mathfrak{l})=\psi_{F}(\mathfrak{l})$ for any prime $\mathfrak{l} \neq \mathfrak{p}$ dividing the conductor of $E / F$,

(4) $L(1, E / F, \delta) \neq 0$.

Thus in order for (6.13) to hold, we are led to

$$
2 \frac{D_{F}^{1 / 2} \mathcal{N}_{F / \mathbb{Q}} \mathfrak{c}_{\delta} L(1, E / F, \delta)}{\tau(\delta)\left(\Omega_{f_{E}}^{\operatorname{sig}\left(\psi_{1}\right)}\right)^{2}} \stackrel{?}{=} 1 \bmod \left(\mathbb{Q}^{\times}\right)^{2}
$$

As a particular case, take $\psi_{1}$ to be trivial. Let $F$ be a real quadratic extension of $\mathbb{Q}$, in which $p$ is inert, and all primes $q \neq p$ dividing the conductor of $E / \mathbb{Q}$ splits in $F$. Let $\delta$ a quadratic Hecke character of $F$, unramified at the primes dividing the conductor of $E / \mathbb{Q}$, such that

(1) $\operatorname{sig}(\delta)=(1,1)$,

(2) $\delta(\mathfrak{p})=-1$,

(3) $\delta(\mathfrak{l})=1$ for any prime $\mathfrak{l} \neq \mathfrak{p}$ dividing the conductor of $E / F$.

Then, is it true that the expression

$$
2 \frac{D_{F}^{1 / 2} \mathcal{N}_{F / \mathbb{Q}} \mathfrak{c}_{\delta} L(1, E / F, \delta)}{\tau(\delta)\left(\Omega_{f_{E}}^{+}\right)^{2}}
$$

is the square of a rational number? This would be consistent with the Birch and Swinnerton-Dyer conjecture. For more on this, see [27]. We remark that for such $\delta$, the restriction $\left.\delta\right|_{\mathbf{A}_{\mathbb{Q}}^{\times}}$is not trivial, so this is not a situation where Waldspurger type results [35] can be directly applied.

Here, we are content to draw the following:

Corollary 6.7. In the situation (6.15), suppose that $E / \mathbb{Q}$ has multiplicative reduction at some prime other than $p$, and that $L^{\prime}\left(1, E / \mathbb{Q}, \psi_{1}\right) \neq 0$ (in addition to the condition $L\left(1, E / \mathbb{Q}, \psi_{2}\right) \neq 0$, and the above hypothesis on $F$ and $\left.\delta\right)$, then the expression (6.15) is the square of a rational number.

Proof. Under the above assumption on $E / \mathbb{Q}$ (in particular that it is multiplicative at some prime other than $p$ ), we have by part (4) of Theorem 5.4 of [4] that

$$
\left.\frac{d}{d k^{2}} L_{p}\left(k / 2, k, f_{\infty}, \psi_{1}\right)\right|_{k=2}=l\left(\log _{E / \mathbb{Q}_{p}} \mathbf{P}^{\prime}\right)^{2}
$$


for some $l \in \mathbb{Q}^{\times}$, with $l=L^{\operatorname{alg}}\left(1, E / \mathbb{Q}, \psi_{2}\right) \bmod \left(\mathbb{Q}^{\times}\right)^{2}$, and $\mathbf{P}^{\prime} \in\left(E\left(\mathbb{Q}^{\psi_{1}}\right) \otimes\right.$ $\mathbb{Q}) \psi_{1}$ non-torsion (which is the conclusion of remark 5.5 applied to $E / \mathbb{Q}$ ). On the other hand, by equation (6.12) and (6.14), we also have

$$
\left.\frac{d}{d k^{2}} L_{p}\left(k / 2, k, f_{\infty}, \psi_{1}\right)\right|_{k=2}=l^{\prime \prime}\left(\log _{E / \mathbb{Q}_{p}} \mathbf{P}\right)^{2}
$$

with $l^{\prime \prime}=l \cdot c$ for some $c \in \mathbb{Q}^{\times}$satisfying

$$
c=2 \frac{D_{F}^{1 / 2} \mathcal{N}_{F / \mathbb{Q}} c_{\delta} L(1, E / F, \delta)}{\tau(\delta)\left(\Omega_{f_{E}}^{\operatorname{sig}\left(\psi_{1}\right)}\right)^{2}} \bmod \left(\mathbb{Q}^{\times}\right)^{2}
$$

and $\mathbf{P} \in\left(E\left(\mathbb{Q}^{\psi_{1}}\right) \otimes \mathbb{Q}\right)_{\psi_{1}}$ again non-torsion. The corollary thus follows from the fact that $\operatorname{dim}_{\mathbb{Q}}\left(E\left(\mathbb{Q}^{\psi_{1}}\right) \otimes \mathbb{Q}\right)_{\psi_{1}}=1$.

To conclude we state another corollary of (6.12), which is a special case of a conjecture of Greenberg (this is stated in [4] in the case when $E / \mathbb{Q}$ is multiplicative at some prime other than $p$ ):

Corollary 6.8. Suppose that $E / \mathbb{Q}$ is split multiplicative at $p$, and $L^{\prime}(1, E / \mathbb{Q}) \neq 0$ (so that the sign of the functional equation of the $p$-adic $L$-function $L_{p}(s, E / \mathbb{Q})$ is $+1)$. Then there exists a $p$-adic neighborhood $U$ of 2 , such that $L\left(k / 2, \mathbf{f}_{k}^{\#}\right) \neq 0$ for $k \in U \cap \mathbb{Z}^{>2}, k \equiv 2 \bmod p-1$.

\section{Appendix I}

Here we prove a result on Gauss sums that is used for the proof of Proposition 5.1 and Lemma 6.1.

In general, let $L$ be a number field. For any infinite place $v$ of $L$, define the modified form of Euler's $\Gamma$ function as follows:

$$
G_{\nu}(s)= \begin{cases}\pi^{-s / 2} \Gamma(s / 2) & \text { if } v \text { is real, } \\ 2(2 \pi)^{-s} \Gamma(s) & \text { if } v \text { is complex. }\end{cases}
$$

Let $\delta$ be a finite order Hecke character of $L$, and $\delta_{v}$ be the local component of $\delta$ at the place $v$. Define

$$
m_{v}(\delta)= \begin{cases}0 & \text { if } v \text { is complex, or } v \text { is real and } \delta_{v}(-1)=1, \\ 1 & \text { if } v \text { is real and } \delta_{v}(-1)=-1\end{cases}
$$

Let $L(s, \delta)$ be the Hecke $L$-function associated to $\delta$. Define the completed $L$ function:

$$
\Lambda(s, \delta)=\left(D_{L} \mathcal{N}_{L / \mathbb{Q}} c_{\delta}\right)^{s / 2} \prod_{\nu \mid \infty} G_{v}\left(s+m_{v}(\delta)\right) L(s, \delta) .
$$


Then $\Lambda(s, \delta)$ satisfies the functional equation

$$
\Lambda(1-s, \delta)=\frac{\tau(\delta)}{i^{M(\delta)} \mathcal{N}_{L / \mathbb{Q}} c_{\delta}^{1 / 2}} \Lambda(s, \delta)
$$

with $M(\delta)=\sum_{v \mid \infty} m_{v}(\delta)$, and $\tau(\delta)$ being the Gauss sum of $\delta$.

If $\delta=1$ is trivial, then $L(s, 1)$ is just the Dedekind zeta function $\zeta_{L}(s)$, and $\Lambda(s, 1)$ is the completed zeta function of $L$. In this case (7.1) reduces to the familiar functional equation:

$$
\Lambda(1-s, 1)=\Lambda(s, 1) .
$$

Now let $F$ be a number field, and $\psi_{1}, \psi_{2}$ be quadratic Hecke characters of $F$, whose conductors are relatively prime to each other. As in Section 3.1, the pair $\psi_{1}, \psi_{2}$ cuts out a quadratic extension $K$ of $F$, and corresponds to a genus character $\psi_{K}$ of $K$. The character $\psi_{K}$ is unramified at all the finite places of $K$, thus $c_{\psi_{K}}=\mathcal{O}_{K}$. On the other hand, let $w$ be an infinite place of $K$ that lies above $v$, then $w$ is real if and only if $m_{v}\left(\psi_{1}\right)=m_{v}\left(\psi_{2}\right)$, in which case there are two real places $w, w^{\prime}$ of $K$ above $v$, with $m_{w}\left(\psi_{K}\right)=m_{w^{\prime}}\left(\psi_{K}\right)=m_{v}\left(\psi_{1}\right)=m_{v}\left(\psi_{2}\right)$. One also sees that

$M\left(\psi_{K}\right)=2 \times$ number of (real) places $v$ of $F$ such that $m_{v}\left(\psi_{1}\right)=m_{v}\left(\psi_{2}\right)=-1$.

Let $\chi$ be a finite order Hecke character of $F$, assumed to be unramified at all the infinite places, so that $m_{v}\left(\psi_{i} \chi\right)=m_{v}\left(\psi_{i}\right)$. For our purpose, we will also assume that $c_{\chi}$ is relatively prime to $c_{\psi_{1}}$ and $c_{\psi_{2}}$, so that $c_{\psi_{i} \chi}=c_{\psi_{i}} c_{\chi}$. Define $\chi_{K}:=\chi \circ \mathcal{N}_{K / F}$.

Proposition 7.1. The following identities hold true:

$$
\begin{aligned}
D_{K} & =D_{F}^{2} \mathcal{N}_{F / \mathbb{Q}} c_{\psi_{1}} c_{\psi_{2}}, \\
\tau\left(\psi_{K} \chi_{K}\right) & =i^{M\left(\psi_{K}\right)-M\left(\psi_{1}\right)-M\left(\psi_{2}\right)} D_{F} D_{K}^{-1 / 2} \tau\left(\psi_{1} \chi\right) \tau\left(\psi_{2} \chi\right) .
\end{aligned}
$$

Proof. We have the following identities:

$$
\begin{gathered}
L\left(s, \psi_{K} \chi_{K}\right)=L\left(s, \psi_{1} \chi\right) L\left(s, \psi_{2}, \chi\right), \\
\left(\pi^{-s / 2} \Gamma(s / 2)\right) \cdot\left(\pi^{-(s+1) / 2} \Gamma((s+1) / 2)\right)=2(2 \pi)^{-s} \Gamma(s)
\end{gathered}
$$

(the second one is the duplication identity for $\Gamma$ function). Hence we obtain

$$
\begin{aligned}
& \Lambda\left(s, \psi_{K} \chi_{K}\right) \\
& \quad=\left(D_{K} \mathcal{N}_{K / \mathbb{Q}} c_{\chi_{K}}\right)^{s / 2}\left(D_{F}^{2} \mathcal{N}_{F / \mathbb{Q}} \mathfrak{c}_{\psi_{1}} \mathcal{N}_{F / \mathbb{Q}} \mathfrak{c}_{\psi_{2}}\right)^{-s / 2} \Lambda\left(s, \psi_{1} \chi\right) \Lambda\left(s, \psi_{2} \chi\right) .
\end{aligned}
$$

On the other hand, by comparing the functional equations for $\Lambda\left(s, \psi_{K} \chi_{K}\right), \Lambda\left(s, \psi_{1} \chi\right)$ and $\Lambda\left(s, \psi_{2} \chi\right)$, we see that the function

$$
F_{\chi}(s):=\left(D_{K} \mathcal{N}_{K / \mathbb{Q}} c_{\chi_{K}}\right)^{s / 2}\left(D_{F}^{2} \mathcal{N}_{F / \mathbb{Q}} \mathfrak{c}_{\psi_{1}} \mathcal{N}_{F / \mathbb{Q}} \mathfrak{c}_{\psi_{2}} \mathcal{N}_{F / \mathbb{Q}} c_{\chi}^{2}\right)^{-s / 2}
$$


satisfies the functional equation

$$
F_{\chi}(1-s)=A_{\chi} \cdot F_{\chi}(s),
$$

where

$$
\begin{aligned}
A_{\chi}=i^{M\left(\psi_{1}\right)+M\left(\psi_{2}\right)-M\left(\psi_{K}\right)} \frac{\tau\left(\psi_{K} \chi_{K}\right)}{\tau\left(\psi_{1} \chi\right) \tau\left(\psi_{2} \chi\right)} \\
\cdot \frac{\left(\mathcal{N}_{F / \mathbb{Q}} c_{\psi_{1}} \mathcal{N}_{F / \mathbb{Q}} c_{\psi_{2}} \mathcal{N}_{F / \mathbb{Q}} c_{\chi}^{2}\right)^{1 / 2}}{\mathcal{N}_{K / \mathbb{Q}} c_{\chi_{K}}^{1 / 2}} .
\end{aligned}
$$

From this, one easily sees that $A_{\chi}$ and $F_{\chi}(s)$ must be identically equal to one.

Take $\chi$ to be trivial. Then from (7.3) we obtain:

$$
D_{K}=D_{F}^{2} \mathcal{N}_{F / \mathbb{Q}} \mathfrak{c}_{\psi_{1}} \mathcal{N}_{F / \mathbb{Q}} \mathfrak{c}_{\psi_{2}}
$$

thus giving the first equation of (7.2). Now going back to general $\chi$, we obtain from (7.3) again:

$$
\mathcal{N}_{K / \mathbb{Q}} c_{\chi_{K}}=\mathcal{N}_{F / \mathbb{Q}} c_{\chi}^{2} .
$$

Plugging these into (7.4), and using $A_{\chi}=1$, we finally obtain the other identity of (7.2).

It is then clear that (6.6) used in the proof of Lemma 6.1 follows from Proposition 7.1 .

For (5.10) used in the proof of Proposition 5.1, we take $\chi$ to be trivial, and $K$ to be a CM field. One has $M\left(\psi_{K}\right)=0$, while $M\left(\psi_{1}\right)+M\left(\psi_{2}\right)=d=[F: \mathbb{Q}]$. Proposition 7.1 implies the claim, by noting that

$$
\tau\left(\psi_{K}\right)=1
$$

which follows since $c_{\psi_{K}}=\mathcal{O}_{K}$.

\section{Appendix II}

Here we prove Proposition 2.9 and Theorem 2.10. The arguments are well-known. We include the proofs here for the convenience of the reader.

Recall the open compact subspace $X^{\prime}$ of $X$ defined by

$$
X^{\prime}=\mathfrak{c} \backslash\left(\mathcal{O}_{F_{\mathfrak{p}}}^{\times} \times p \mathcal{O}_{F_{\mathfrak{p}}}\right)
$$

which is stable under $I_{\mathfrak{p}}$ (as in Section $2.4, \mathfrak{c}$ is the closure in $\mathcal{O}_{F_{\mathfrak{p}}}^{\times}$of the group of totally positive units of $\mathcal{O}_{F}$ ). Let $\mathcal{D}^{\prime}$ be the space of $\mathbb{C}_{p}$-valued measures on $X^{\prime}$, with 
the evident structure of a module over $\widetilde{\Lambda}_{F}$ and the action of $I_{\mathfrak{p}}$. As in the case of $\mathfrak{D}_{*}$, one has maps

$$
\mathbb{D}^{\prime} \stackrel{s^{\prime}}{\rightarrow} \mathscr{D} \stackrel{p^{\prime}}{\rightarrow} \mathscr{D}^{\prime}
$$

defined in the evident manner, and allow us to define, for $\mu^{\prime} \in \mathscr{D}^{\prime}$, and $g \in$ $M_{2}\left(\mathfrak{p} ; \mathcal{O}_{F_{\mathfrak{p}}}\right) \cap \mathrm{GL}_{2}\left(F_{\mathfrak{p}}\right)$,

$$
g \star \mu^{\prime}:=p^{\prime}\left(g \cdot s^{\prime}\left(\mu^{\prime}\right)\right) .
$$

One checks that this defines an action of the semigroup $M_{2}\left(\mathfrak{p} ; \mathcal{O}_{F_{\mathfrak{p}}}\right) \cap \mathrm{GL}_{2}\left(F_{\mathfrak{p}}\right)$ on $D^{\prime}$ extending that of $I_{\mathfrak{p}}$. Thus for $f$ a function on $X^{\prime}$, and $S \subset X^{\prime}$ open compact

$$
\int_{\mathcal{S}} f d\left(g \star \mu^{\prime}\right)=\int_{g^{-1}(\mathcal{S}) \cap X^{\prime}} f \mid g d \mu^{\prime} .
$$

The measure $g \star \mu^{\prime}$ is supported on $g\left(X^{\prime}\right) \cap X^{\prime}$.

Consider the map

$$
\mathscr{D}_{*} \rightarrow \mathscr{D}^{\prime}
$$

given by restriction of measures. This map is $I_{\mathfrak{p}}$-equivariant.

Let $\Sigma=\Sigma\left(\mathfrak{n}^{+}, \mathfrak{n}^{-}\right), \quad \Sigma^{\prime}=\Sigma\left(\mathfrak{n}^{+}, \mathfrak{n}^{-}\right) \cap \Sigma\left(\mathfrak{p}, \mathfrak{n}^{-}\right)=\Sigma\left(\mathfrak{p} \mathfrak{n}^{+}, \mathfrak{n}^{-}\right)$. Thus $\Sigma_{\mathfrak{p}}^{\prime}=I_{\mathfrak{p}}$. The restriction map (8.1) induces the $I_{\mathfrak{p}}$-equivariant map

$$
S\left(\Sigma, \mathscr{D}_{*}\right) \rightarrow S\left(\Sigma^{\prime}, \mathscr{D}^{\prime}\right) .
$$

It is clear that the specialization map (2.10) factors through (8.2).

First, we observe:

Proposition 8.1. The map (8.2) is an isomorphism.

Proof. For $a \in k_{\mathfrak{p}}$, denote by $\gamma_{a}=\left(\begin{array}{ll}a & 1 \\ 1 & 0\end{array}\right)=\left(\begin{array}{cc}1 & a \\ 0 & 1\end{array}\right)\left(\begin{array}{ll}0 & 1 \\ 1 & 0\end{array}\right) \in \mathrm{GL}_{2}\left(\mathcal{O}_{F_{\mathfrak{p}}}\right)$.

Then one can check that

$$
\mathcal{O}_{F_{\mathfrak{p}}} \times \mathcal{O}_{F_{\mathfrak{p}}}^{\times}=\bigsqcup_{a \in k_{\mathfrak{p}}} \gamma_{a}\left(\mathcal{O}_{F_{\mathfrak{p}}}^{\times} \times p \mathcal{O}_{F_{\mathfrak{p}}}\right)
$$

Denote $\tilde{X}:=X \backslash X^{\prime}=\mathfrak{c} \backslash\left(\mathcal{O}_{\mathfrak{p}} \times \mathcal{O}_{F_{\mathfrak{p}}}^{\times}\right)$. Then

$$
\tilde{X}=\bigsqcup_{a \in k_{\mathfrak{p}}} \gamma_{a} X^{\prime}
$$


Now given $\Phi^{\prime} \in S\left(\Sigma^{\prime}, D^{\prime}\right)$, suppose for the moment that $\Phi \in S\left(\Sigma, D_{*}\right)$ is a form that maps to $\Phi^{\prime}$ under (8.2). Then for any continuous function $f$ on $X$,

$$
\begin{aligned}
\int_{X} f d \Phi(g) & =\int_{X^{\prime}} f d \Phi(g)+\int_{\widetilde{X}} f d \Phi(g) \\
& =\int_{X^{\prime}} f d \Phi(g)+\sum_{a \in k_{\mathfrak{p}}} \int_{\gamma_{a} X^{\prime}} f d \Phi(g) \\
& =\int_{X^{\prime}} f d \Phi(g)+\sum_{a \in k_{\mathfrak{p}}} \int_{X^{\prime}} f \mid \gamma_{a} d \Phi\left(g \cdot \gamma_{a}\right) \\
& =\int_{X^{\prime}} f d \Phi^{\prime}(g)+\sum_{a \in k_{\mathfrak{p}}} \int_{X^{\prime}} f \mid \gamma_{a} d \Phi^{\prime}\left(g \cdot \gamma_{a}\right) .
\end{aligned}
$$

Thus in general, given $\Phi^{\prime} \in S\left(\Sigma^{\prime}, D^{\prime}\right)$ define $\Phi$ by the following rule: for any continuous function $f$ on $X$,

$$
\int_{X} f d \Phi(g)=\int_{X^{\prime}} f d \Phi^{\prime}(g)+\sum_{a \in k_{\mathfrak{p}}} \int_{X^{\prime}} f \mid \gamma_{a} d \Phi^{\prime}\left(g \cdot \gamma_{a}\right) .
$$

Then one checks that $\Phi \in S\left(\Sigma, D_{*}\right)$, and this provides the inverse to the map (8.2).

The isomorphism $S\left(\Sigma, D_{*}\right) \rightarrow S\left(\Sigma^{\prime}, D^{\prime}\right)$ commutes with the action of the Hecke operators $T_{\mathfrak{I}}$ and $T_{\mathfrak{I}, \mathfrak{I}}$ for $\mathfrak{l}$ not dividing $\mathfrak{p} \mathfrak{n}^{+} \mathfrak{n}^{-}$and the $U_{\mathfrak{I}}$ for $\mathfrak{l} \mid \mathfrak{n}^{+} \mathfrak{n}^{-}$. For the Hecke operators at $\mathfrak{p}$ we have:

Proposition 8.2. The isomorphism (8.2) intertwines the action of $T_{\mathfrak{p}}$ on $S\left(\Sigma, D_{*}\right)$ and that of $U_{\mathfrak{p}}$ on $S\left(\Sigma^{\prime}, D^{\prime}\right)$.

Proof. This is a direct computation. Thus let $\Phi \in S\left(\Sigma, \mathscr{D}_{*}\right)$, and let $\Phi^{\prime}$ be the image of $\Phi$ under the isomorphism (8.2).

Given a continuous function $f$ on $X$ we have, for $g \in \widehat{B}^{\times}$, the following formula for $T_{\mathfrak{p}} \Phi$ (equation (2.2)): by taking

$$
\sigma_{a}=\left(\begin{array}{cc}
p & a \\
0 & 1
\end{array}\right) \quad \text { for } a \in k_{\mathfrak{p}}
$$

and

$$
\sigma_{\infty}=\left(\begin{array}{ll}
1 & 0 \\
0 & p
\end{array}\right)
$$

we have

$$
\mathrm{GL}_{2}\left(\mathcal{O}_{F_{\mathfrak{p}}}\right)\left(\begin{array}{ll}
p & 0 \\
0 & 1
\end{array}\right) \mathrm{GL}_{2}\left(\mathcal{O}_{F_{\mathfrak{p}}}\right)=\bigsqcup_{a \in \mathbf{P}^{1}\left(k_{\mathfrak{p}}\right)} \sigma_{a} \mathrm{GL}_{2}\left(\mathcal{O}_{F_{\mathfrak{p}}}\right) .
$$


Hence

$$
\begin{aligned}
\int_{X} f d T_{\mathfrak{p}} \Phi(g) & =\int_{X} f d \sigma_{\infty}(\mathfrak{p}) \star \Phi\left(g \sigma_{\infty}\right)+\sum_{a \in k_{\mathfrak{p}}} \int_{X} f d \sigma_{a} \star \Phi\left(g \sigma_{a}\right) \\
& =\int_{X^{\prime}} f d \sigma_{\infty} \star \Phi\left(g \sigma_{\infty}\right)+\sum_{a \in k_{\mathfrak{p}}} \int_{\tilde{X}} f d \sigma_{a} \star \Phi\left(g \sigma_{a}\right)
\end{aligned}
$$

(in the last equality we are using the fact that the measures $\sigma_{\infty} \star \Phi\left(g \sigma_{\infty}\right)$ and $\sigma_{a} \star$ $\Phi\left(g \sigma_{a}\right)$ are supported on $X^{\prime}$ and $\tilde{X}$ respectively).

Whereas in general for $\Psi \in S\left(\Sigma^{\prime}, D^{\prime}\right)$, and function $h$ on $X^{\prime}$, we have (equation (2.3)) that

$$
\begin{aligned}
\int_{X^{\prime}} h d U_{\mathfrak{p}} \Psi(g) & =\sum_{b \in k_{\mathfrak{p}}} \int_{X^{\prime}} h d \hat{\sigma}_{b} \star \Psi\left(g \sigma_{b}\right) \\
& =\sum_{b \in k_{\mathfrak{p}}} \int_{\hat{\sigma}_{b}^{-1} X^{\prime} \cap X^{\prime}} h \mid \hat{\sigma}_{b} d \Psi\left(g \hat{\sigma}_{b}\right) \\
& =\sum_{b \in k_{\mathfrak{p}}} \int_{X^{\prime}} h \mid \hat{\sigma}_{b} d \Psi\left(g \hat{\sigma}_{b}\right) .
\end{aligned}
$$

Here

$$
\hat{\sigma}_{b}=\left(\begin{array}{cc}
1 & 0 \\
b p & p
\end{array}\right) \quad \text { for } b \in k_{\mathfrak{p}}
$$

One has

$$
\mathcal{O}_{F_{\mathfrak{p}}}^{\times} \times p \mathcal{O}_{F_{\mathfrak{p}}}=\bigsqcup_{b \in k_{\mathfrak{p}}} \hat{\sigma}_{b}\left(\mathcal{O}_{F_{\mathfrak{p}}}^{\times} \times p \mathcal{O}_{F_{\mathfrak{p}}}\right)
$$

which implies

$$
X^{\prime}=\bigsqcup_{b \in k_{\mathfrak{p}}} \hat{\sigma}_{b} X^{\prime}
$$

(In particular $\hat{\sigma}_{b}^{-1} X^{\prime} \supset X^{\prime}$, explaining the last equality in the above computation.)

Now suppose that $\widetilde{\Phi} \in S\left(\Sigma, D_{*}\right)$ corresponds to $U_{\mathfrak{p}}\left(\Phi^{\prime}\right)$ under the isomorphism (8.2). Then as we have seen in the proof of Proposition 8.1, for $f$ a continuous function on $X$,

$$
\int_{X} f d \widetilde{\Phi}(g)=\int_{X^{\prime}} f d U_{\mathfrak{p}} \Phi^{\prime}(g)+\sum_{a \in k_{\mathfrak{p}}} \int_{X^{\prime}} f \mid \gamma_{a} d U_{\mathfrak{p}} \Phi^{\prime}\left(g \gamma_{a}\right)
$$


Now

$$
\begin{aligned}
\int_{X^{\prime}} f d U_{\mathfrak{p}} \Phi^{\prime}(g) & =\sum_{b \in k_{\mathfrak{p}}} \int_{X^{\prime}} f \mid \hat{\sigma}_{b} d \Phi^{\prime}\left(g \hat{\sigma}_{b}\right) \\
& =\sum_{b \in k_{\mathfrak{p}}} \int_{X^{\prime}} f \mid \hat{\sigma}_{b} d \Phi\left(g \hat{\sigma}_{b}\right) \\
& =\sum_{b \in k_{\mathfrak{p}}} \int_{\hat{\sigma}_{b} X^{\prime}} f d \hat{\sigma}_{b} \star \Phi\left(g \hat{\sigma}_{b}\right) .
\end{aligned}
$$

Notice that $\hat{\sigma}_{b}=\sigma_{\infty} \tau_{b}$, where $\tau_{b}=\left(\begin{array}{ll}1 & 0 \\ b & 1\end{array}\right) \in \operatorname{GL}_{2}\left(\mathcal{O}_{F_{\mathfrak{p}}}\right)$. Hence

$$
\hat{\sigma}_{b} \star \Phi\left(g \sigma_{b}\right)=\sigma_{\infty} \star \Phi\left(g \sigma_{\infty}\right) .
$$

So we have

$$
\begin{aligned}
\int_{X^{\prime}} f d U_{\mathfrak{p}} \Phi^{\prime}(g) & =\sum_{b \in k_{\mathfrak{p}}} \int_{\hat{\sigma}_{b} X^{\prime}} f d \sigma_{\infty} \star \Phi\left(g \sigma_{\infty}\right) \\
& =\int_{X^{\prime}} f d \sigma_{\infty} \star \Phi\left(g \sigma_{\infty}\right) .
\end{aligned}
$$

Similarly we can treat the other terms:

$$
\begin{aligned}
\int_{X^{\prime}} f \mid \gamma_{a} d U_{\mathfrak{p}} \Phi^{\prime}\left(g \gamma_{a}\right) & =\sum_{b \in k_{\mathfrak{p}}} \int_{X^{\prime}} f \mid \gamma_{a} \hat{\sigma}_{b} d \Phi^{\prime}\left(g \gamma_{a} \hat{\sigma}_{b}\right) \\
& =\sum_{b \in k_{\mathfrak{p}}} \int_{X^{\prime}} f \mid \gamma_{a} \hat{\sigma}_{b} d \Phi\left(g \gamma_{a} \hat{\sigma}_{b}\right) .
\end{aligned}
$$

Now a direct computation gives

$$
\gamma_{a} \hat{\sigma}_{b}=\sigma_{a} \gamma_{b}
$$

hence the above integral becomes

$$
\begin{aligned}
\sum_{b \in k_{\mathfrak{p}}} \int_{X^{\prime}} f \mid \sigma_{a} \gamma_{b} d \Phi\left(g \sigma_{a} \gamma_{b}\right) & =\sum_{b \in k_{\mathfrak{p}}} \int_{\gamma_{b} X^{\prime}} f \mid \sigma_{a} d \Phi\left(g \sigma_{a}\right) \\
& =\int_{\tilde{X}} f \mid \sigma_{a} d \Phi\left(g \sigma_{a}\right) \\
& =\int_{\tilde{X}} f d \sigma_{a} \star \Phi\left(g \sigma_{a}\right) .
\end{aligned}
$$


The last equality holds because $\sigma_{a}^{-1}(\tilde{X}) \cap X=\tilde{X}$. So we have

$$
\int_{X} f d \widetilde{\Phi}(g)=\int_{X} f d T_{\mathfrak{p}} \Phi(g) .
$$

Thus we conclude that $\widetilde{\Phi}=T_{\mathfrak{p}} \Phi$.

Now let $\Phi_{\infty}^{\prime} \in S\left(\Sigma^{\prime}, \mathscr{D}^{\prime}\right)$. For integer $k \geq 2$, let $\Phi_{k}=\rho_{k, *}\left(\Phi_{\infty}^{\prime}\right)$. Then

\section{Lemma 8.3.}

$$
\rho_{k, *}\left(U_{\mathfrak{p}} \Phi_{\infty}^{\prime}\right)=U_{\mathfrak{p}} \Phi_{k}
$$

Proof. Let $P \in \mathscr{B}_{k}$. Then for $g \in \widehat{B}^{\times}$,

$$
\rho_{k, *}\left(U_{\mathfrak{p}} \Phi_{\infty}^{\prime}\right)(g)(P)=\int_{X^{\prime}} f_{P} d U_{\mathfrak{p}} \Phi_{\infty}^{\prime}(g)
$$

(where $f_{P}$ is as in equation (2.9)). On the other hand, as we saw in equation (8.3) during the proof of Proposition 8.2,

$$
\int_{X^{\prime}} f_{P} d U_{\mathfrak{p}} \Phi_{\infty}^{\prime}(g)=\sum_{a \in k_{\mathfrak{p}}} \int_{X^{\prime}} f_{P} \mid \hat{\sigma}_{a} d \Phi_{\infty}^{\prime}\left(g \hat{\sigma}_{a}\right) .
$$

Hence

$$
\begin{aligned}
\rho_{k, *}\left(U_{\mathfrak{p}} \Phi_{\infty}^{\prime}\right)(g)(P) & =\sum_{a \in k_{\mathfrak{p}}} \int_{X^{\prime}} f_{P} \mid \hat{\sigma}_{a} d \Phi_{\infty}^{\prime}\left(g \hat{\sigma}_{a}\right) \\
& =\sum_{a \in k_{\mathfrak{p}}} \Phi_{k}\left(g \hat{\sigma}_{a}\right)\left(P \mid \hat{\sigma}_{a}\right) \\
& =U_{\mathfrak{p}}\left(\Phi_{k}\right)(g)(P) .
\end{aligned}
$$

Corollary 8.4 (Proposition 2.9). Assume that $\Phi_{\infty} \in S\left(\Sigma, \mathcal{D}_{*}\right)$, and $\Phi_{k}=\rho_{k, *}\left(\Phi_{\infty}\right)$. Then

$$
\rho_{k, *}\left(T_{\mathfrak{p}} \Phi_{\infty}\right)=U_{\mathfrak{p}} \Phi_{k}
$$

Proof. Let $\Phi_{\infty}^{\prime} \in S\left(\Sigma^{\prime}, D^{\prime}\right)$ be the image of $\Phi_{\infty}$ under the isomorphism (8.2). Then by Proposition 8.2 ,

$$
T_{\mathfrak{p}} \Phi_{\infty}=U_{\mathfrak{p}} \Phi_{\infty}^{\prime}
$$

Thus using Lemma 8.3

$$
\rho_{k, *}\left(T_{\mathfrak{p}}\left(\Phi_{\infty}\right)\right)=\rho_{k, *}\left(U_{\mathfrak{p}}\left(\Phi_{\infty}^{\prime}\right)\right)=U_{\mathfrak{p}}\left(\rho_{k, *} \Phi_{\infty}^{\prime}\right)=U_{\mathfrak{p}}\left(\Phi_{k}\right) .
$$


We draw another corollary of Proposition 8.1:

Corollary 8.5. The specialization map

$$
\rho_{2, *}: S\left(\Sigma, D_{*}\right) \rightarrow S\left(\Sigma^{\prime}, V_{2}\right)
$$

is surjective.

Proof. By Proposition 8.1 this amounts to showing that the specialization map

$$
\rho_{2, *}: S\left(\Sigma^{\prime}, \mathscr{D}^{\prime}\right) \rightarrow S\left(\Sigma^{\prime}, V_{2}\right)
$$

is surjective.

Let $R^{\prime}$ be the Eichler order of $B$ of level $\mathfrak{p} \mathfrak{n}^{+}$such that $\Sigma^{\prime}=\left(\widehat{R}^{\prime}\right)^{\times}$. Choose a set of representatives $\left\{t_{i}\right\}_{i=1}^{n} \in \widehat{B}^{\times}$for the double coset $B^{\times} \backslash \widehat{B}^{\times} / \Sigma^{\prime}$. For each $i$ let $R_{i}^{\prime}:=B \cap t_{i} \hat{R}^{\prime} t_{i}^{-1}$ be the Eichler order of $B$ of level $\mathfrak{p} \mathfrak{n}^{+}$determined by $t_{i}$. Then each of the quotient $\left(R_{i}^{\prime}\right)^{\times} / \mathcal{O}_{F,+}^{\times}$is a finite group (recall that $\mathcal{O}_{F,+}^{\times}$is the group of totally positive units of $\left.\mathcal{O}_{F}\right)$. Let $\Delta_{i}$ be the image of $t_{i}^{-1}\left(R_{i}^{\prime}\right)^{\times} t_{i} / \mathcal{O}_{F,+}^{\times}$in $\Sigma_{\mathfrak{p}}^{\prime} / \mathrm{c}$. Then in general for any $\mathbb{Z}_{p}\left[\Sigma_{\mathfrak{p}}\right]$-module $M$ with the action of $\Sigma_{\mathfrak{p}}$ on $M$ factors through $\Sigma_{\mathfrak{p}} / \mathfrak{c}$ there is a bijection of $\mathbb{Z}_{p}$-modules:

$$
\begin{aligned}
S\left(\Sigma^{\prime}, M\right) & \stackrel{\sim}{\longrightarrow} \bigoplus_{i=1}^{n} M^{\Delta_{i}} \\
\Phi & \longmapsto\left\{\Phi\left(t_{i}\right)\right\}_{i=1}^{n}
\end{aligned}
$$

(here $M^{\Delta_{i}}$ stands for the $\mathbb{Z}_{p}$-submodule of elements of $M$ invariant under $\Delta_{i}$ ). So it suffices to show that the maps $\rho_{2}:\left(\mathscr{D}_{*}^{\prime}\right)^{\Delta_{i}} \rightarrow\left(V_{2}\right)^{\Delta_{i}}$ are surjective for $i=1, \ldots, n$. But the $\Delta_{i}$ are finite groups, and $\mathscr{D}^{\prime}$ is a $\mathbb{Q}_{p}$-vector space, so this follows from the surjectivity of $\rho_{2}: \mathscr{D}^{\prime} \rightarrow V_{2}=\mathbb{C}_{p}$.

Remark 8.6. For each $k \geq 2$ the same argument shows, by using the surjectivity of $\rho_{k}: \mathscr{D}^{\prime} \rightarrow V_{k}$, that $\rho_{k, *}: S\left(\Sigma, D_{*}\right) \rightarrow\left(\Sigma^{\prime}, V_{k}\right)$ is surjective.

Proof of Theorem 2.10. This can be proved by the same methods as in [1] and [21] (see also [2]), so we just provide a sketch. Let $\Phi \in S\left(\Sigma^{\prime}, V_{2}\right)=S\left(\Sigma\left(\mathfrak{p}, \mathfrak{n}^{+}, \mathfrak{n}^{-}\right), V_{2}\right)$ be an eigenform that is new at primes dividing $\mathfrak{n}^{+}$. Then there is a finite extension $E / \mathbb{Q}_{p}$ such that $\Phi$ takes values in $E$. Multiplying $\Phi$ by scalars if necessary, we can further assume that $\Phi$ takes values in $\mathcal{O}=\mathcal{O}_{E}$ (the ring of integers of $E$ ). Denote by $S\left(\Sigma^{\prime}, V_{2}, \mathcal{O}\right)$ the $\mathcal{O}$-module of forms with values in $\mathcal{O}$, which is a finite free module over $\mathcal{O}$. Similarly let $\mathcal{D}_{*, \mathcal{O}}$ be the $\mathcal{O}$-module of measures on $X$ with values in $\mathcal{O}$. Then $S\left(\Sigma, \mathcal{D}_{*, \mathcal{O}}\right)$ is a module over $\widetilde{\Lambda}_{F, \mathcal{O}}:=\mathcal{O} \otimes_{\mathbb{Z}_{p}} \widetilde{\Lambda}_{F}$. The ring $\widetilde{\Lambda}_{F, \mathcal{O}}$ is compact, and the $\widetilde{\Lambda}_{F, \mathcal{O}}$-modules $\mathscr{D}_{*, \mathcal{O}}$ and $S\left(\Sigma, \mathcal{D}_{*, \mathcal{O}}\right)$ are compact. 
The specialization map $\rho_{2, *}$ sends $S\left(\Sigma, D_{*, \mathcal{O}}\right)$ to $S\left(\Sigma^{\prime}, V_{2}, \mathcal{O}\right)$. As in [1], Section 5, let $S\left(\Sigma^{\prime}, V_{2}, \mathcal{O}\right)^{0}$ and $S\left(\Sigma, \mathscr{D}_{*, \mathcal{O}}\right)^{0}$ be the ordinary parts of $S\left(\Sigma^{\prime}, V_{2}, \mathcal{O}\right)$ and $S\left(\Sigma, D_{*, \mathcal{O}}\right)$, which is the maximal direct summand (as $\mathcal{O}$-module and $\widetilde{\Lambda}_{F, \mathcal{O}}$-module respectively) over which the action of $T_{\mathfrak{p}}$ is invertible (as usual $T_{\mathfrak{p}}$ acts as $U_{\mathfrak{p}}$ on $\left.S\left(\Sigma^{\prime}, V_{2}, \mathcal{O}\right)\right)$. Thus $\Phi \in S\left(\Sigma^{\prime}, V_{2}, \mathcal{O}\right)^{0}$. The arguments used for the proof of Theorem 5.1 of [1] (known as control type theorems) show that $\rho_{2, *}$ induces an injection

$$
\rho_{2, *}: S\left(\Sigma, \mathscr{D}_{*, \mathcal{O}}\right)^{0} / P_{2} S\left(\Sigma, D_{*, \mathcal{O}}\right)^{0} \hookrightarrow S\left(\Sigma^{\prime}, V_{2}, \mathcal{O}\right)^{0}
$$

here we identify the weight $P_{2} \in \mathfrak{X}_{F}$ of Section 2.4 as the prime ideal of $\tilde{\Lambda}_{F, \mathcal{O}}$ given by the kernel of $P_{2}$. The compact version of Nakayama's lemma implies that $S\left(\Sigma, \mathscr{D}_{*, \mathcal{G}}\right)^{0}$ is a finite $\widetilde{\Lambda}_{F, \mathcal{O}}$-module. In particular $\subseteq:=S\left(\Sigma, \mathscr{D}_{*, \mathcal{O}}\right)^{0} \otimes_{\tilde{\Lambda}_{F, \mathcal{O}}} \widetilde{\Lambda}_{\mathbb{Q}, \mathcal{O}}$ is finite over $\tilde{\Lambda}_{\mathbb{Q}, \mathcal{O}}$, hence finite over $\Lambda_{\mathcal{O}}:=\mathcal{O} \otimes_{\mathbb{Z}_{p}} \Lambda$. The argument of [1], Lemma 1.1, shows that $\mathbb{S}$ is torsion free over $\Lambda_{\mathcal{O}}$.

On the other hand, the proof of Corollary 8.5 shows that $\rho_{2, *}: S\left(\Sigma, \mathscr{D}_{*, \mathcal{O}}\right) \otimes_{\mathcal{O}}$ $E \rightarrow S\left(\Sigma^{\prime}, V_{2}, \mathcal{O}\right) \otimes_{\mathcal{O}} E$ is surjective, so without loss of generality we may assume that $\Phi$ lies in the image of $S\left(\Sigma, D_{*, \mathcal{O}}\right)$, hence in the image of $S\left(\Sigma, D_{*, \mathcal{O}}\right)^{0}$ since $\Phi$ is ordinary. Let $\Phi_{\infty}^{\prime} \in \mathcal{S}$ be such that $\rho_{2, \infty}\left(\Phi_{\infty}^{\prime}\right)=\Phi$.

Let $\mathfrak{P}$ be the prime ideal of $\Lambda_{\mathcal{O}}$ given by the kernel of the evaluation map at $k=2$. The localization $\widetilde{S}_{\mathfrak{\beta}}$ is then finite and torsion-free over the discrete valuation ring $\Lambda_{\mathcal{O}, \Re}$, hence is finite free over $\Lambda_{\mathcal{O}, \Re}$. Let $\Re$ be the $\Lambda_{\mathcal{O}, \Re \text {-subalgebra }}$ of $\operatorname{End}_{\Lambda_{\mathcal{O}, \mathfrak{B}}}\left(\widetilde{S}_{\mathfrak{B}}\right)$ generated by the image of the Hecke algebra $\mathbf{T}$ (with $\mathbf{T}$ as in Section 2.5). The algebra $\Re$ is finite free over $\Lambda_{\mathcal{O}, \mathfrak{\beta}}$. The system of Hecke eigenvalues associated to the eigenform $\Phi$ then gives rise to an algebra homomorphism $h: \Re \rightarrow E$, and the kernel of $h$ is a maximal ideal of $\Re$ that lies over $\mathfrak{P} \Lambda_{\mathcal{O}, \mathfrak{\Re}}$. Let $\mathcal{I}$ be the normalization of the quotient of $\Re$ by the minimal prime of $\Re$ contained in $\operatorname{ker}(h)$ (which is again a DVR). Since $\Phi$ is new at primes dividing $\mathfrak{n}^{+}$, the arguments used to prove Theorem 3.6 of [21] (p. 381-383 of loc. cit.) show that $\mathcal{I}$ is unramified over $\Lambda_{\mathcal{O}, \Re}$ (see especially the second equation on p. 383 of [21]). Fix an embedding $\mathcal{I} / m_{\mathcal{I}} \rightarrow \overline{\mathbb{Q}}_{p}$ (where $m_{\mathcal{I}}$ is the maximal ideal of $\mathfrak{I}$ ). Then since $\mathcal{I}$ is unramified over $\Lambda_{\mathcal{O}, \Re}$, there is an unique way to embed $\mathcal{I}$ into $\Lambda^{\dagger}$ extending the embedding of $\Lambda_{\mathcal{O}, \mathfrak{\beta}}$ into $\Lambda^{\dagger}$ (as in equation (2.5)).

As in loc. cit. there is a decomposition of $\Lambda_{\mathcal{O}, \Re}$ algebras

$$
\Re \otimes_{\Lambda_{\mathcal{O}, \Re}} \Lambda^{\dagger} \cong \Lambda^{\dagger} \times \mathcal{K},
$$

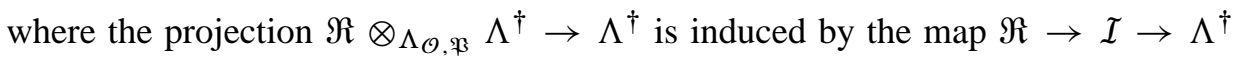
(here $\mathcal{K}$ is just the complimentary algebra direct summand).

Let $e_{\mathcal{I}} \in \Re \otimes_{\Lambda_{\mathcal{O}, \mathfrak{B}}} \Lambda^{\dagger}$ be the idempotent corresponding to the projection onto the $\Lambda^{\dagger}$. Under the natural pairing

$$
\Re \otimes_{\Lambda_{\mathcal{O}, \mathfrak{\beta}}} \Lambda^{\dagger} \times \widetilde{S}_{\mathfrak{P}} \longrightarrow \widetilde{S}_{\mathfrak{P}} \otimes_{\Lambda_{\mathcal{O}, \mathfrak{\beta}}} \Lambda^{\dagger}=\subseteq \otimes_{\Lambda_{\mathcal{O}}} \Lambda^{\dagger}
$$


put $\Phi_{\infty}:=e_{\mathcal{I}} \Phi_{\infty}^{\prime} \in \mathfrak{S} \otimes_{\Lambda_{\mathcal{O}}} \Lambda^{\dagger}$. Then $\Phi_{\infty}$ is an eigenform in $\mathfrak{S} \otimes_{\Lambda_{\mathcal{O}}} \Lambda^{\dagger}$. The image of $\Phi_{\infty}$ in $S\left(\Sigma, \mathcal{D}_{*}^{\text {cycl, } \dagger}\right)$ is then an eigenform that specializes to $\Phi$.

\section{References}

[1] A. Ash and G. Stevens, $p$-adic deformations of cohomology classes of subgroups of $\mathrm{GL}_{n}(\mathbb{Z})$. Collect. Math. 48 (1997), 1-30. Zbl 0866.20038 MR 1464013

[2] B. Balasubramanyam and M. Longo, $\Lambda$-adic modular symbols over totally real fields. Comment. Math. Helv. 86 (2011), 841-865.

[3] M. Bertolini and H. Darmon, Heegner points, $p$-adic $L$-functions, and the CerednikDrinfeld uniformization. Invent. Math. 131 (1998), no. 3, 453-491. Zbl 0899.11029 MR 1614543

[4] M. Bertolini and H. Darmon, Hida families and rational points on elliptic curves. Invent. Math. 168 (2007), no. 2, 371-431. Zbl 1129.11025 MR 2289868

[5] M. Bertolini and H. Darmon, The rationality of Stark-Heegner points over genus fields of real quadratic fields. Ann. of Math. (2) 170 (2009), no. 1, 343-370. Zbl 1203.11045 MR 2521118

[6] J. Boutot and H. Carayol, Uniformisation $p$-adique des courbes de Shimura: les théorèmes de Cerednik et de Drinfeld. In Corubes modulaires et courbes de Shimura (Orsay, 1987/1988), Astérisque 196-197(7) (1991-1992), 45-158. Zbl 0781.14010 MR 1141456

[7] J. F. Boutot and T. Zink, The $p$-adic uniformisation of Shimura curves. Preprint 95-107, Sonderforschungsbereich 343, Universität Bielefeld, 1995.

[8] C. Breuil, B. Conrad, F. Diamond and R. Taylor, On the modularity of elliptic curves over Q: wild 3-adic exercises. J. Amer. Math. Soc. 14 (2001), no. 4, 843-939. Zbl 0982.11033 MR 1839918

[9] D. Bump, Automorphic forms and representations. Cambridge Stud. Adv. Math. 55, Cambridge University Press, Cambridge 1997. Zbl 0868.11022 MR 1431508

[10] H. Carayol, Sur la mauvaise réduction des courbes de Shimura. Compositio Math. 59 (1986), no. 2, 151-230. Zbl 0607.14021 MR 0860139

[11] A. Dabrowski, $p$-adic $L$-functions of Hilbert modular forms. Ann. Inst. Fourier 44 (1994), no. 4, 1025-1041. Zbl 0808.11035 MR 1306548

[12] H. Darmon, Rational points on modular elliptic curves. CBMS Regional Conf. Ser. Math. 101, Amer. Math. Soc., Providence, RI, 2004. Zbl 1057.11034 MR 2020572

[13] S. Dasgupta, Gross-Stark units, Stark-Heegner points, and class fields of real quadratic fields. PhD thesis, University of California-Berkeley, 2004.

[14] S. Dasgupta and J. Teitelbaum, The $p$-adic upper half plane. In p-adic geometry, Univ. Lecture Ser. 45, Amer. Math. Soc., Providence, RI, 2008, 65-121. Zbl 1153.14021 MR 2482346

[15] S. Friedberg and J. Hoffstein, Nonvanishing theorems for automorphic $L$-functions on GL(2). Ann. of Math. 142 (1995), 385-423. Zbl 0847.11026 MR 1343325 
[16] R. Greenberg and G. Stevens, $p$-adic $L$-functions and $p$-adic periods of modular forms. Invent. Math. 111 (1993), no. 2, 407-447. Zbl 0778.11034 MR 1198816

[17] R. Greenberg and G. Stevens, On the conjecture of Mazur, Tate, and Teitelbaum. In p-adic monodromy and the Birch and Swinnerton-Dyer conjecture, Contemp. Math. 165, Amer. Math. Soc., Providence, RI, 1994, 183-211. Zbl 0846.11030 MR 1279610

[18] B. Gross, Heights and the special values of $L$-series. In Number Theory (Montreal), CMS Conf. Proc. 7, 1987, 115-187. Zbl 0623.10019 MR 0894322

[19] B. Gross, Heegner points and representation theory. In Heegner points and Rankin Lseries, Math. Sci. Res. Inst. Publ., 49, Cambridge University Press, Cambridge 2004, 37-65. Zbl 1126.11032 MR 2083210

[20] R. Hatcher, Heights and L-series. Canad. J. Math. 42 (1990), no. 3, 533-560. Zbl 0723.11024 MR 1062744

[21] H. Hida, On $p$-adic Hecke algebras for $\mathrm{GL}_{2}$ over totally real fields. Ann. of Math. (2) 128 (1988), no. 2, 295-384. Zbl 0658.10034 MR 0960949

[22] H. Hida, H. Hida, On nearly ordinary Hecke algebras for GL(2) over totally real fields. In Algebraic number theory, Adv. Stud. Pure Math. 17, Academic Press, Boston, Mass., 1989, 139-169. Zbl 0742.11026 MR 1097614

[23] B. Howard, Twisted Gross-Zagier theorems. Canad. J. Math. 61 (2009), no. 4, 828-887. Zbl 05585517 MR 2541387

[24] V. Kolyvagin and D. Logachev, Finiteness of III over totally real fields. Izv. Akad. Nauk SSSR Ser. Mat. 55 (1991), no. 4, 851-876. Zbl 0791.14019 MR 1137589

[25] B. Mazur, J. Tate and J. Teitelbaum, On $p$-adic analogues of the conjectures of Birch and Swinnerton-Dyer. Invent. Math. 84 (1986), no.1, 1-48. Zbl 0699.14028 MR 0830037

[26] C. P. Mok, The exceptional zero conjecture for Hilbert modular forms. Compositio Math. 145 (2009), 1-55. Zbl 05532998 MR 2480494

[27] C. P. Mok, Special values of $L$-functions of elliptic curves over $\mathbf{Q}$ and their base change to real quadratic fields. J. Number Theory 130 (2010), 431-438. Zbl 05655003 MR 2564905

[28] D. Rohrlich, On $L$-functions of elliptic curves and cyclotomic towers. Invent. Math. 75 (1984), no. 3, 409-423. Zbl 0565.14006 MR 0735333

[29] D. Rohrlich, L-functions and division towers. Math. Ann. 281 (1988), 611-632. Zbl 0656.14013 MR 0958262

[30] G. Shimura, The special values of the zeta functions associated with cusp forms. Comm. Pure Appl. Math. 29 (1976), no. 6, 783-804. Zbl 0348.10015 MR 0434962

[31] G. Shimura, The special values of the zeta functions associated with Hilbert modular forms. In Collected Papers, Volume III, No. 78c (the original version appeared in Duke Math. J. 45 (1978), no.3, 637-679). Zbl 0394.10015 MR 0507462

[32] R. Taylor and A. Wiles, Ring-theoretic properties of certain Hecke algebras. Ann. of Math. (2) 141 (1995), no. 3, 553-572. Zbl 0823.11030 MR 1333036

[33] Y. Varshavsky, $p$-adic uniformization of unitary Shimura varieties. II. J. Differential Geom. 49 (1998), no. 1, 75-113. Zbl 0923.11092 MR 1642109

[34] M.-F. Vigneras, Arithmétique des algèbres de quaternions. Lecture Notes in Math. 800, Springer-Verlag, Berlin 1980. Zbl 0422.12008 MR 0580949 
[35] J.-L. Waldspurger, Sur les valeurs de certaines fonctions $L$ automorphes en leur centre de symétrie. Compositio Math. 54 (1985), no. 2, 173-242. Zbl 0567.10021 MR 0783511

[36] A. Wiles, On ordinary $\lambda$-adic representations associated to modular forms. Invent. Math. 94 (1988), 529-573. Zbl 0664.10013 MR 0969243

[37] A. Wiles, Modular elliptic curves and Fermat's Last Theorem. Ann. of Math. 141 (1995), 443-551. Zbl 0823.11029 MR 1333035

[38] H. Xue, Central values of Rankin L-series. Internat. Math. Res. Notices 2006 (2006), 1-41. Zbl 1141.11028 MR 2249999

[39] H. P. Yuan, Special value formulae of Rankin-Selberg $L$-functions. Ph.D. thesis, University of Pennsylvania, 2005.

[40] S. W. Zhang, Heights of Heegner points on Shimura curves. Ann. of Math. (2) 153 (2001), 27-147. Zbl 1036.11029 MR 1826411

[41] S. W. Zhang, Gross-Zagier formula for $\mathrm{GL}_{2}$. Asian J. Math. 5(2) (2001), 183-290. Zbl 1111.11030 MR 1868935

Received April 4, 2009

Chung Pang Mok, Department of Mathematics \& Statistics, McMaster University, 1280 Main Street West, Hamilton, Ontario L8S 4K1, Canada

E-mail: cpmok@math.mcmaster.ca 